\title{
Proceedings of the Workshop for Applied Nuclear Data: WANDA2020
}

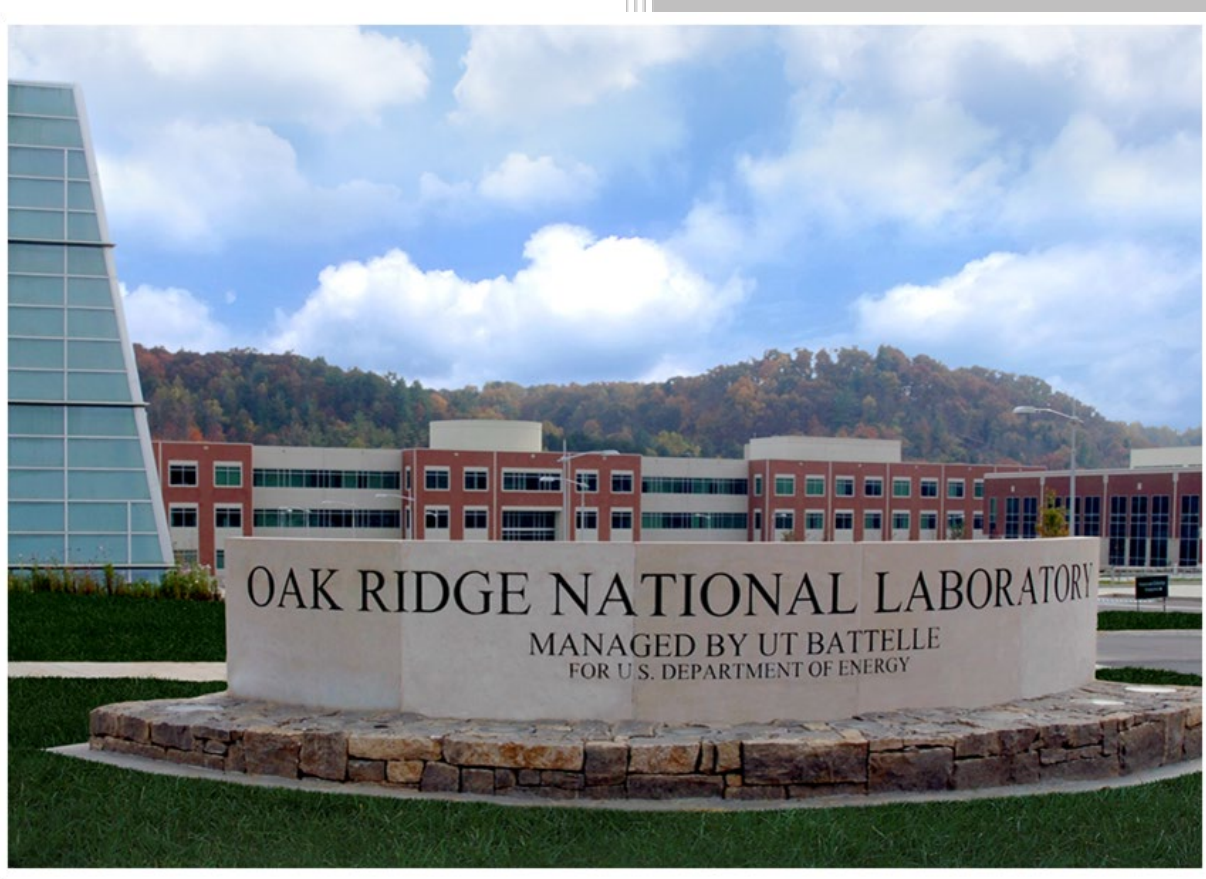

\section{Approved for public release.} Distribution is unlimited.
Catherine Romano Lee Bernstein Teresa Bailey Friederike Bostelmann David Brown Robert Casperson Jeremy Conlin Yaron Danon Matthew Devlin Bethany Goldblum Michael Grosskopf Amanda Lewis Denise Neudecker Ellen O'Brien Bruce Pierson Brian Quiter Andrew Ratkiewicz Gregory Severin Michael S. Smith Vladimir Sobes Alejandro Sonzogni Patrick Talou Fredrik Tovesson Etienne Vermeulen Kyle Wendt Michael Zerkle 


\section{DOCUMENT AVAILABILITY}

Reports produced after January 1, 1996, are generally available free via US Department of Energy (DOE) SciTech Connect.

Website www.osti.gov

Reports produced before January 1, 1996, may be purchased by members of the public from the following source:

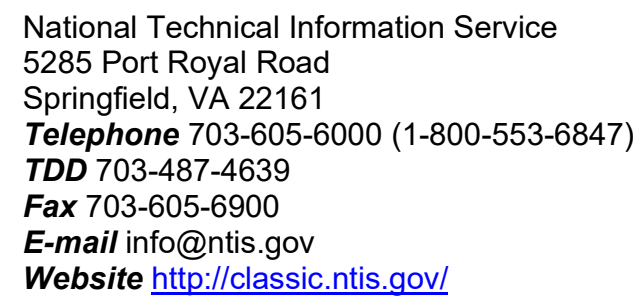

Reports are available to DOE employees, DOE contractors, Energy Technology Data Exchange representatives, and International Nuclear Information System representatives from the following source:

Office of Scientific and Technical Information

PO Box 62

Oak Ridge, TN 37831

Telephone 865-576-8401

Fax 865-576-5728

E-mail reports@osti.gov

Website http://www.osti.gov/contact.html

This report was prepared as an account of work sponsored by an agency of the United States Government. Neither the United States Government nor any agency thereof, nor any of their employees, makes any warranty, express or implied, or assumes any legal liability or responsibility for the accuracy, completeness, or usefulness of any information, apparatus, product, or process disclosed, or represents that its use would not infringe privately owned rights. Reference herein to any specific commercial product, process, or service by trade name, trademark, manufacturer, or otherwise, does not necessarily constitute or imply its endorsement, recommendation, or favoring by the United States Government or any agency thereof. The views and opinions of authors expressed herein do not necessarily state or reflect those of the United States Government or any agency thereof. 
Reactor and Nuclear Systems Division

\title{
PROCEEDINGS OF THE WORKSHOP FOR APPLIED NUCLEAR DATA: WANDA2020
}

${ }^{1}$ Catherine Romano, ${ }^{2,6}$ Lee Bernstein, ${ }^{4}$ Teresa Bailey, ${ }^{1}$ Friederike Bostelmann, ${ }^{3}$ David Brown, ${ }^{12}$ Yaron Danon, ${ }^{4}$ Robert Casperson, ${ }^{5}$ Jeremy Conlin, ${ }^{5}$ Matthew Devlin, ${ }^{6}$ Bethany Goldblum, ${ }^{5}$ Michael Grosskopf, ${ }^{6}$ Amanda Lewis, ${ }^{5}$ Denise Neudecker, ${ }^{5}$ Ellen O'Brien, ${ }^{7}$ Bruce Pierson, ${ }^{2}$ Brian Quiter, ${ }^{4}$ Andrew Ratkiewicz, ${ }^{8}$ Gregory Severin, ${ }^{1}$ Michael S. Smith, ${ }^{9}$ Vladimir Sobes, ${ }^{3}$ Alejandro Sonzogni, ${ }^{5}$ Patrick Talou, ${ }^{10}$ Fredrik Tovesson, ${ }^{5}$ Etienne Vermeulen, ${ }^{4}$ Kyle Wendt, ${ }^{11}$ Michael Zerkle

\footnotetext{
${ }^{1}$ Oak Ridge National Laboratory

${ }^{2}$ Lawrence Berkeley National Laboratory

${ }^{3}$ Brookhaven National Laboratory

${ }^{4}$ Lawrence Livermore National Laboratory

${ }^{5}$ Los Alamos National Laboratory

${ }^{6}$ University of California-Berkeley

${ }^{7}$ Pacific Northwest National Laboratory

${ }^{8}$ Michigan State University

${ }^{9}$ University of Tennessee-Knoxville

${ }^{10}$ Argonne National Laboratory

${ }^{11}$ Naval Nuclear Laboratory

${ }^{12}$ Rensselaer Polytechnic Institute
}

Date Published: July 23, 2020

\author{
Prepared by \\ OAK RIDGE NATIONAL LABORATORY \\ Oak Ridge, TN 37831-6283 \\ managed by \\ UT-BATTELLE, LLC \\ for the \\ US DEPARTMENT OF ENERGY \\ under contract DE-AC05-00OR22725
}





\section{TABLE OF CONTENTS}

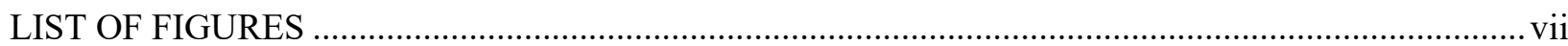

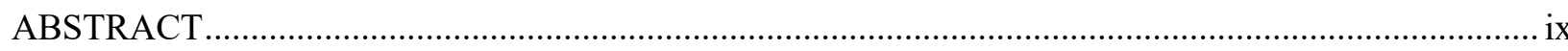

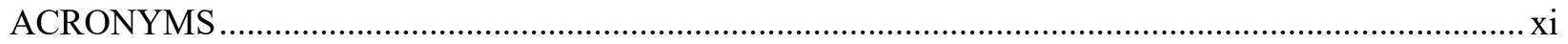

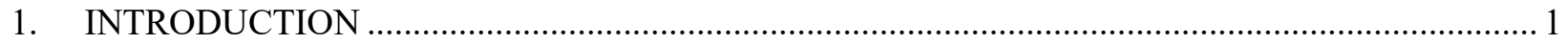

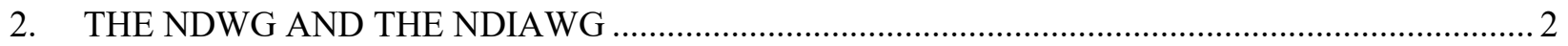

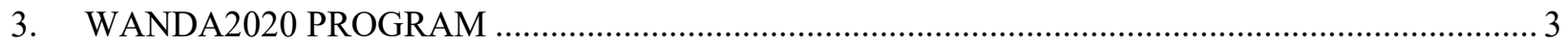

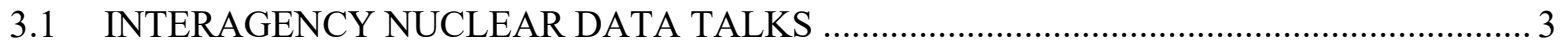

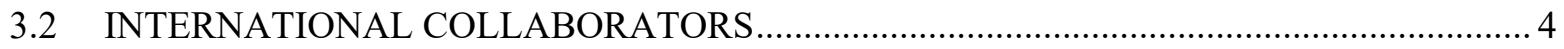

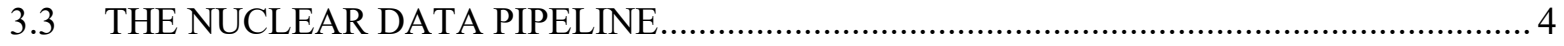

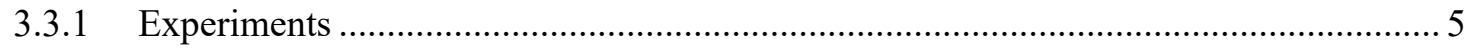

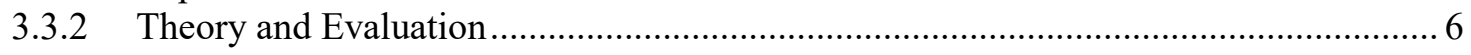

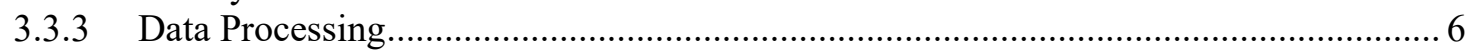

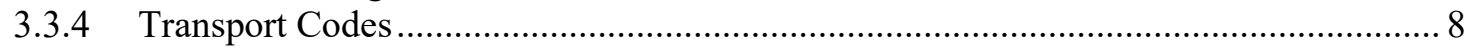

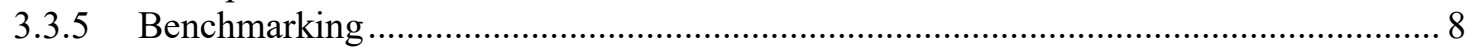

3.3.6 Users, Sensitivity, Uncertainty and Setting Priorities ........................................... 9

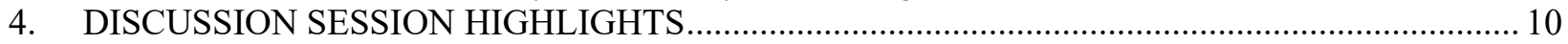

4.1 MACHINE LEARNING AND ARTIFICIAL INTELLIGENCE FOR NUCLEAR

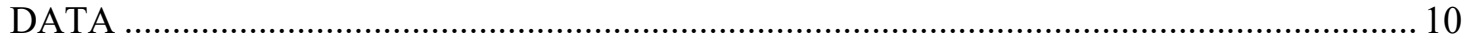

4.2 DETECTOR MODELS, ATOMIC DATA, AND STOPPING POWERS ............................ 10

4.3 COVARIANCE/ SENSITIVITY/ VALIDATION SESSION …........................................... 11

4.4 NUCLEAR DATA FOR ISOTOPE PRODUCTION AND NUCLEAR DATA

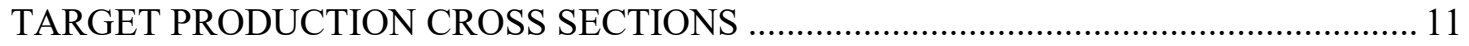

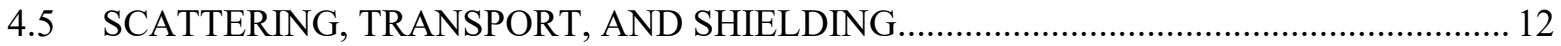

4.6 NEUTRON-INDUCED GAMMA PRODUCTION AND GAMMA DECAY ….................. 12

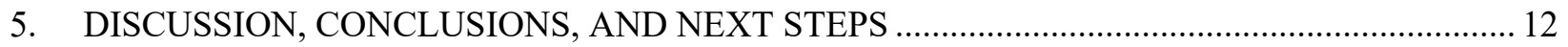

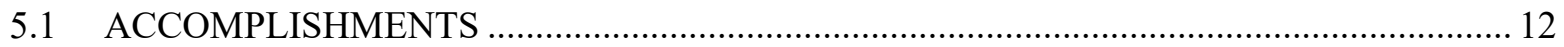

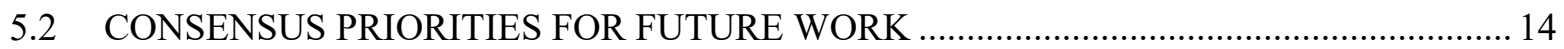

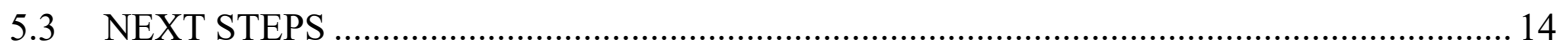

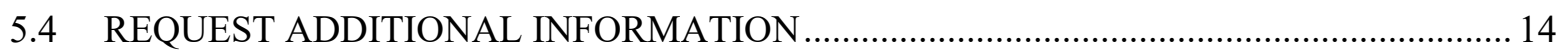

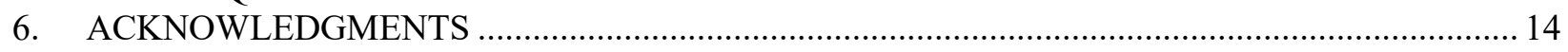

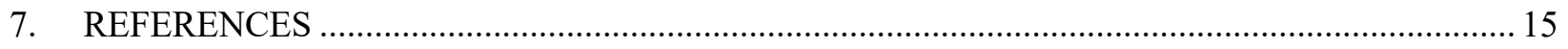

APPENDIX A. ROADMAPPING SESSION SUMMARIES …................................................. A-1

A.1 Machine Learning and Artificial Intelligence for Nuclear Data ...................................... A-2

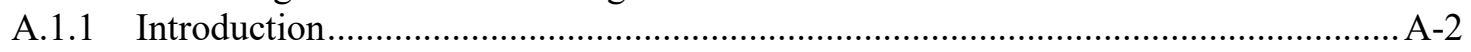

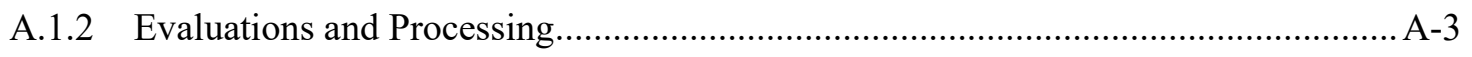

A.1.3 Experiments / Compilations ............................................................................ A-3

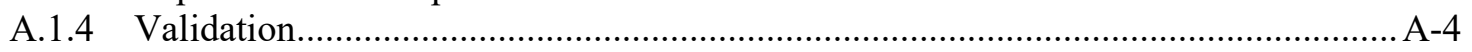

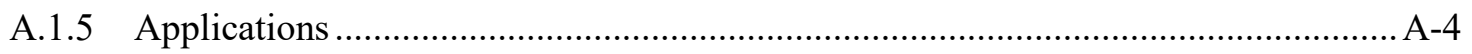

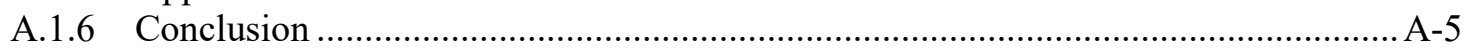

A.2 Detector Models, Atomic Data, and Stopping Powers ….............................................. A-6

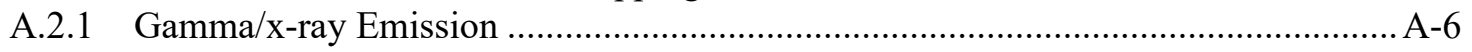

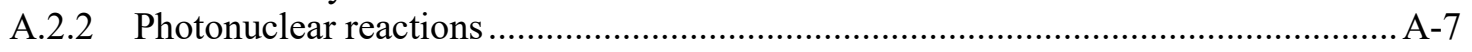

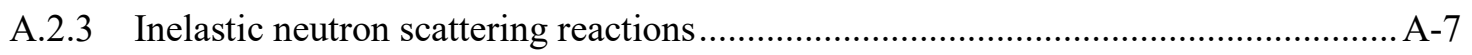

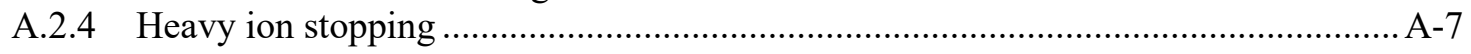

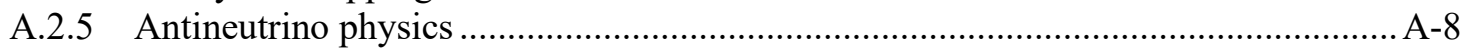

A.2.6 Neutron response of organic scintillators........................................................... A-8

A.2.7 Experiment and modeling integration................................................................. A-9

A.3 Covariance/ Sensitivity/ Validation Session .................................................................. A-10 


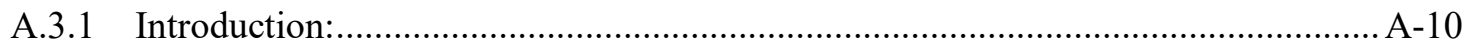

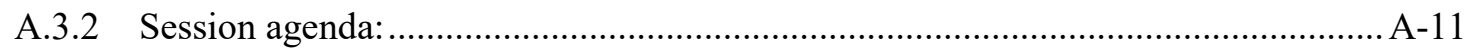

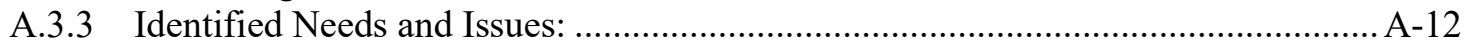

A.3.3.1 Differential experimental data covariance needs: ................................... A-12

A.3.3.2 Evaluated covariances needs: ....................................................... A-12

A.3.3.3 Propagation to applications/ validation: .................................................. A-13

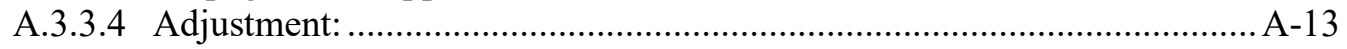

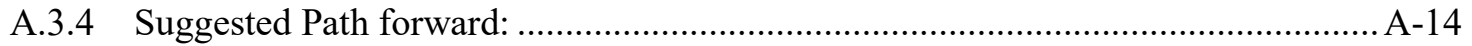

A.3.4.1 Addressing differential experimental data covariance needs ................... A-14

A.3.4.2 Evaluated covariances needs ............................................................. A-14

A.3.4.3 Propagation to applications/ validation: .............................................. A-15

A.3.4.4 Adjustment: ................................................................................... A-15

A.4 Nuclear Data for Isotope Production and Nuclear Data Target Production Cross

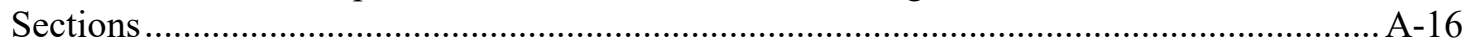

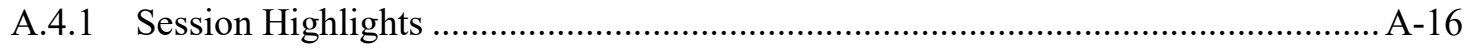

A.4.1.1 Department of Energy Isotope Program (DOE IP) ............................... A-16

A.4.1.2 International Atomic Energy Agency (IAEA) .................................... A-17

A.4.2 Nuclear Data Capabilities and Needs................................................................ A-18

A.4.2.1 Lawrence Berkeley National Laboratory (LBNL) ................................. A-18

A.4.2.2 Facility for Rare Isotope Beams (FRIB) at Michigan State University

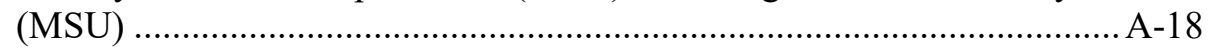

A.4.2.3 Oak Ridge National Laboratory (ORNL) ............................................... A-19

A.4.2.4 Argonne National Laboratory (ANL) .................................................. A-20

A.4.2.5 Los Alamos National Laboratory (LANL) ........................................... A-21

A.4.3 Nuclear Data Target Production Capabilities and Needs..................................... A-21

A.4.3.1 Lawrence Berkeley National Laboratory (LBNL) .................................. A-22

A.4.3.2 Oak Ridge National Laboratory (ORNL) .......................................... A-22

A.4.3.3 Argonne National Laboratory (ANL) ................................................ A-22

A.4.3.4 Los Alamos National Laboratory (LANL) .......................................... A-23

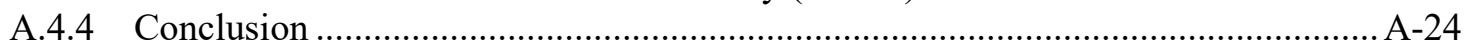

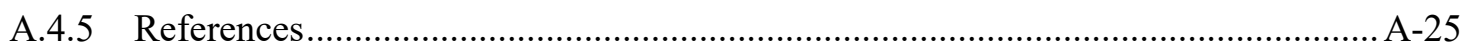

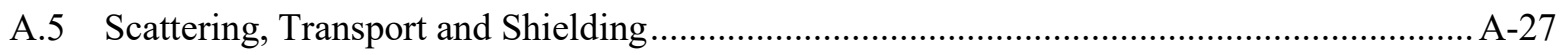

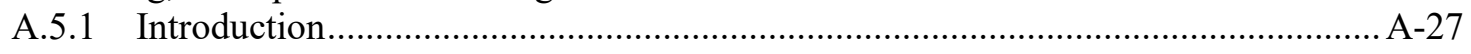

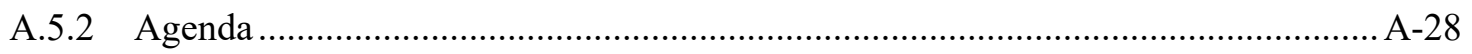

A.5.3 Session One Highlights - Neutron Transport Codes: .......................................... A-28

A.5.4 Session Two Highlights - Experiments to Improve Nuclear Data: ........................ A-29

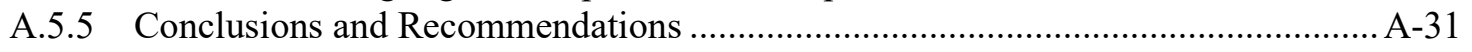

A.6 Neutron Induced Gamma Production and Gamma Decay ……....................................... A-32

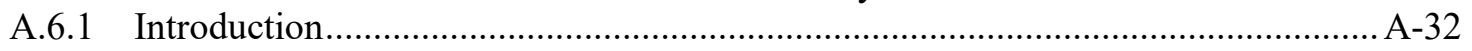

A.6.2 Experimental Facilities Overview.................................................................... A-32

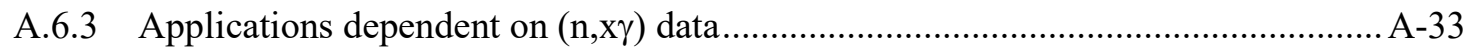

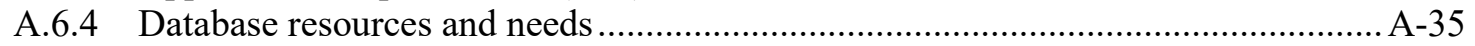

A.6.5 The Path Forward: A Coordinated Measurement and Evaluation Program.............A-38

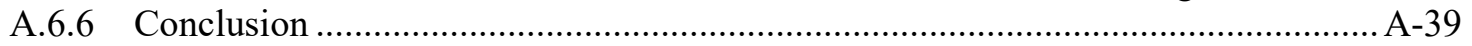

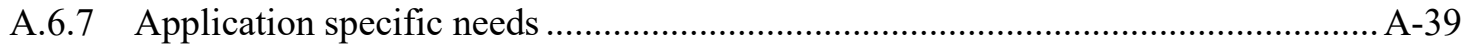

A.6.7.1 Nuclear Data Needs for Oil Well Logging (M. Mauborgne) .................... A-40

A.6.7.2 Nuclear Data Needs for NASA Dragonfly/BECA mission (M. Ayllon-Unzueta)........................................................................... A-41

APPENDIX B. MEETING AGENDA AND PRESENTATIONS .................................................... B-1 


\section{LIST OF FIGURES}

Figure 1. Collaborative goals of the WANDA workshops. ............................................................ 2

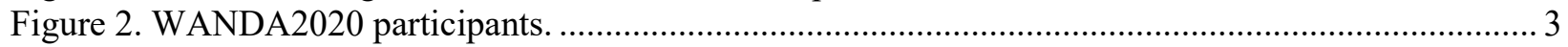

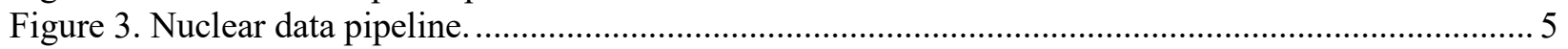

Figure 4. Prompt neutron multiplicity data used for the determination of the ENDF/B-VIII.0 value

Figure 5. Computed/experimental criticality $k_{\text {eff }}$ values for a test suite of LEU-COMP-THERM experiments.

Figure A.1. Neutron flux at the various facilities presented by Bleuel.

A-32

Figure A.2. Examples of nuclear data deficiencies in the modeling of the time-dependent gammaray signal from pulsed neutron scattering using a $14 \mathrm{MeV}$ DT-API source using MCNPPoliMi (left) and GEANT 4 (right) from the talk by McConchie.

Figure A.3. Data for the production of the $4.4 \mathrm{MeV} \gamma$-ray in ${ }^{12} \mathrm{C}$ from the ${ }^{16} \mathrm{O}(\mathrm{n}, \mathrm{n}$ ' $\alpha \gamma)$ reaction (left) and the $1.7 \mathrm{MeV} \gamma$-ray from ${ }^{27} \mathrm{Al}\left(\mathrm{n}, \mathrm{n}^{\prime} \gamma\right.$ ) (right) from the talk by Ayllon. (What is the big ? supposed to represent?)

Figure A.4. Comparison between measured and modeled ${ }^{56} \mathrm{Fe}\left(\mathrm{n}, \mathrm{n}^{\prime} \gamma\right)$ transitions for the yrast $2^{+} \rightarrow$ $0^{+}$(left) and a more highly excited off-yrast $4^{+} \rightarrow$ yrast $2^{+}$transition (right) highlighting the importance of accurate deformation and spin-parity assignments.

Figure A.5. Goodness of fit between the ${ }^{56} \mathrm{Fe}\left(\mathrm{n}, \mathrm{n}^{\prime} \gamma\right)$ data in the Atlas and data from and energy differential cross section measurement from GELINA convolved with and exponential and a Maxwell-Boltzmann neutron spectrum.

Figure A.6. A comparison measured and modeled values for total photon energy and multiplicity from ${ }^{235} \mathrm{U}(\mathrm{n}, \mathrm{f})$. 



\begin{abstract}
On March 3-5, 2020, in Washington, DC, the Nuclear Data Interagency Working Group (NDIAWG) supported the 3-day Workshop for Applied Nuclear Data (WANDA2020) to facilitate interagency collaboration on nuclear data for applications and to communicate mission-driven nuclear data needs. The goal was to assemble users and producers of nuclear data to provide input to identify nuclear data needs, suggest solutions to address those needs, and prioritize potential solutions. The workshop consisted of talks by agency program managers, a nuclear data pipeline panel, six topic-focused roadmapping discussion sessions and a review of NDIAWG-funded projects. More than 160 attendees represented national laboratories, universities, and headquarters, as well as international organizations and industry. The proceedings presented herein summarize the workshop's content, highlight important outcomes, and document attendees' recommendations.
\end{abstract}




\section{ACRONYMS}

\begin{tabular}{|c|c|}
\hline $\mathrm{AI} / \mathrm{ML}$ & artificial intelligence/machine learning \\
\hline AMPX & a modern cross section processing system \\
\hline ANL & Argonne National Laboratory \\
\hline BNL & Brookhaven National Laboratory \\
\hline CGMF & Upgrade to CGM for fission \\
\hline CRP & coordinated research project \\
\hline CSEWG & Cross Section Evaluation Working Group \\
\hline DNN R\&D & Office of Defense Nuclear Nonproliferation R\&D \\
\hline DOE & US Department of Energy \\
\hline DT & fusion of a deuterium and a tritium atom \\
\hline DTRA & Defense Threat Reduction Agency \\
\hline EGAF & evaluated gamma activation file \\
\hline ENDF & evaluated nuclear data file \\
\hline ENSDF & evaluated nuclear structure data file \\
\hline EXFOR & experimental nuclear reaction data \\
\hline FOA & funding opportunity announcement \\
\hline FRIB & Facility for the Production of Rare Isotope Beams \\
\hline GEANT & Software package for Geometry and Tracking \\
\hline GNDS & General Nuclear Data Structure \\
\hline IAEA & International Atomic Energy Agency \\
\hline ICSBEP & International Handbook of Evaluated Criticality Safety Benchmark Experiments \\
\hline INL & Idaho National Laboratory \\
\hline IRPhEP & International Handbook of Evaluated Reactor Physics Benchmark Experiments \\
\hline KERMA & Kinetic Energy Released in Material \\
\hline LANL & Los Alamos National Laboratory \\
\hline LANSCE & Los Alamos Neutron Science Center \\
\hline LBNL & Lawrence Berkeley National Laboratory \\
\hline LENS & low-energy nuclear science \\
\hline LEU-COMP-THERM & low enriched uranium-composite-thermal spectrum \\
\hline LINAC & Linear Accelerator \\
\hline LLNL & Lawrence Livermore National Laboratory \\
\hline MCNP & Monte Carlo N-Particle \\
\hline NDEM & Nuclear Data Exchange Meeting \\
\hline NDIAWG & Nuclear Data Interagency Working Group \\
\hline NDNCA & Nuclear Data Needs and Capabilities for Applications \\
\hline NDREW & Nuclear Data Roadmapping and Enhancement Workshop \\
\hline NDWG & Nuclear Data Working Group \\
\hline $\mathrm{NE}$ & Office of Nuclear Energy \\
\hline NJOY & nuclear data processing code \\
\hline NNDC & National Nuclear Data Center \\
\hline NNSA & National Nuclear Security Administration \\
\hline NP & Office of Nuclear Physics \\
\hline ORNL & Oak Ridge National Laboratory \\
\hline PNNL & Pacific Northwest National Laboratory \\
\hline R\&D & research and development \\
\hline RPI & Rensselaer Polytechnic Institute \\
\hline SINBAD & Shielding Integral Benchmark Archive and Database \\
\hline TALYS & software for the simulation of nuclear reactions \\
\hline TENDL & TALYS-based Evaluated Nuclear Data Library \\
\hline WANDA2020 & Workshop for Applied Nuclear Data 2020 \\
\hline WPEC & Working Party on International Nuclear Data Evaluation Cooperation \\
\hline
\end{tabular}




\section{INTRODUCTION}

The Workshop for Applied Nuclear Data (WANDA2020) is the fourth in a series of nuclear data workshops focused on nuclear data for applications and their impact to those applications. The first of these workshops was the Nuclear Data Needs and Capabilities for Applications (NDNCA) workshop hosted by the DOE Office of Nuclear Physics (DOE NP) and Office of Defense Nuclear Nonproliferation Research and Development (DNN R\&D) at Lawrence Berkeley National Laboratory (LBNL) in 2015 [1]. The workshop targeted needs for all applications and achieved its goal of collecting expert opinions from users on a broad range of nuclear data needs. The NDNCA created a strong foundation for future discussions. The published list of nuclear data needs includes several cross-cutting areas in which multiple users can benefit from data improvements. As a result of NDNCA, the Nuclear Data Working Group (NDWG) was formed to facilitate cross-program collaboration and was made up of representatives designated by program offices with an interest in nuclear data cooperation [2]. The NDWG identified and prioritized several of the most important cross-cutting nuclear data needs and presented a proposed solution, as well as general recommendations for funding nuclear data, to 25 federal program representatives at the Nuclear Data Exchange Meeting (NDEM) on April 15, 2016, in Washington, DC. The NDEM provided an opportunity for critical conversations between the nuclear data community and program managers to provide guidance in resolving nuclear data needs.

After the NDEM, a group of interested federal program managers created the Nuclear Data Interagency Working Group (NDIAWG) chaired by DOE NP to coordinate nuclear data funding between participating program offices. The result was the 2017 NDIAWG funding opportunity announcement (FOA), which was managed by DOE NP for all programs [3]. This FOA became the first of an annual NDIAWG nuclear data FOA $[4,5]$. Since the first FOA, the following projects have been funded:

1. Improving the Nuclear Data on Fission Product Decays at CARIBU

2. Novel Approach for Improving Antineutrino Spectral Predictions for Nonproliferation Applications

3. ${ }^{238} \mathrm{U}(\mathrm{p}, \mathrm{xn})$ and ${ }^{235} \mathrm{U}(\mathrm{d}, \mathrm{xn}){ }^{235-237} \mathrm{~Np}$ Nuclear Reaction Cross Sections Relevant to the Production of ${ }^{2369} \mathrm{~Np}$

4. State-of-the-art Gamma-ray Spectroscopy to Enhance the ENSDF

5. Beta-strength Function, Reactor Decay Heat, and Anti-neutrino Properties from Total Absorption Spectroscopy of Fission Fragments

6. Improving the Double-differential ${ }^{238} \mathrm{U}\left(\mathrm{n}, \mathrm{n}^{\prime} \mathrm{g}\right)$ Cross Section Using Neutron-gamma Coincidences

7. Integral Measurements of Independent and Cumulative Fission Product Yields Supporting Nuclear Forensics and Other Applications

8. Evaluation of Energy Dependent Fission Product Yields

9. Measurement of Independent Fission Product Yields

10. Independent Fission Product Yields from 0.5 to $20 \mathrm{MeV}$

11. Energy Dependent Fission Product Yields

12. Nonproliferation Nuclear Data Scoping Studies

The scope of the FOAs is informed by annual workshops organized by the NDWG. The Nuclear Data Roadmapping and Enhancement Workshop in January 2018 [6] focused on nonproliferation, and WANDA2019 [7] focused on cross-cutting nuclear data topics. WANDA2020 also focused on crosscutting nuclear data. These workshops were intended to achieve the following objectives:

1. To facilitate communication and collaboration among programs and organizations dependent on nuclear data. 
2. To collect subject matter expert input, including nuclear data prioritization and recommended solutions.

3. To increase mutual awareness and understanding of different stakeholder segments of the nuclear data community, including experimentalists, evaluators, end users, and program managers.

4. To provide guidance for the priorities called out in the annual NDIAWG FOA as well as other agency calls for proposals.

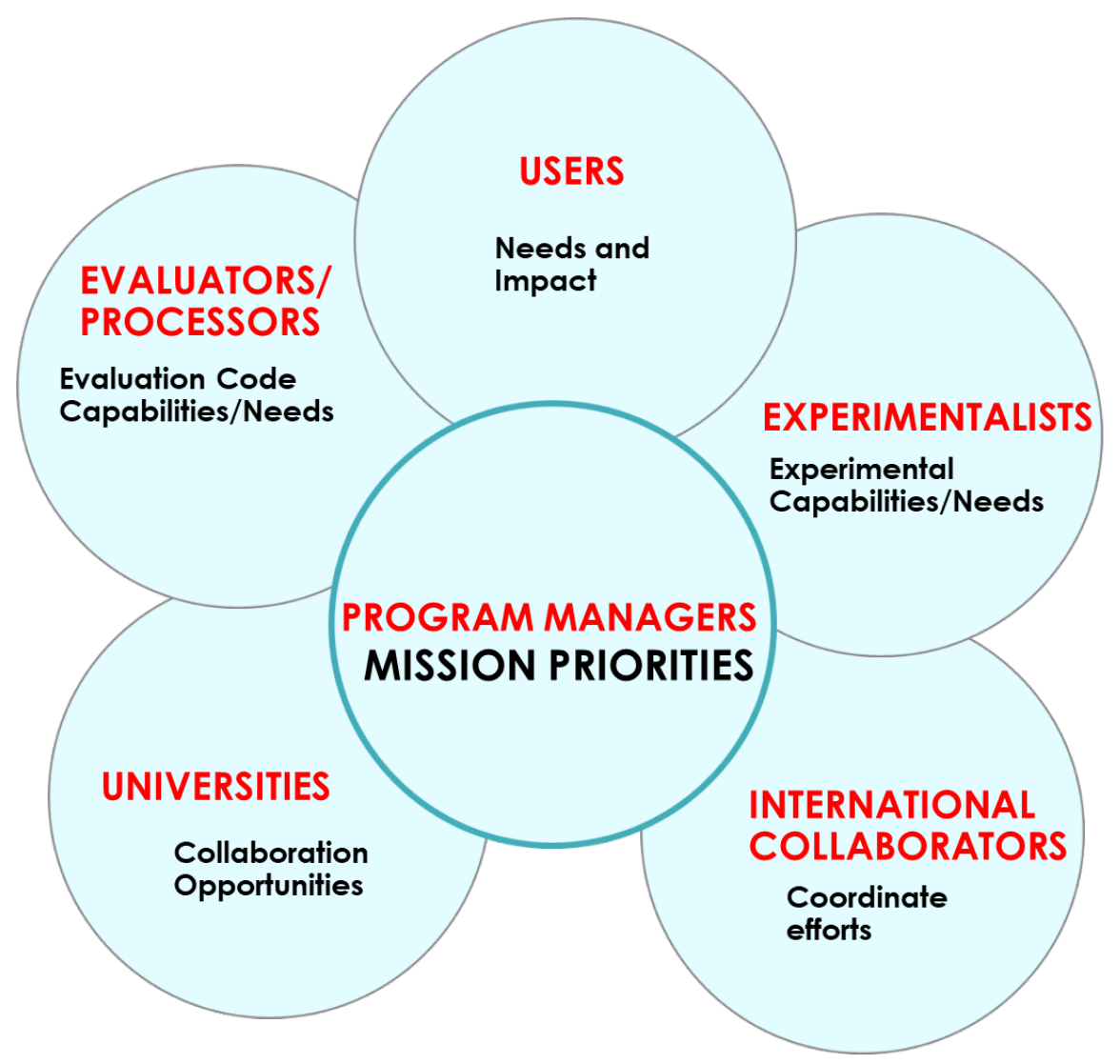

Figure 1. Collaborative goals of the WANDA workshops.

\section{THE NDWG AND THE NDIAWG}

The NDWG was formed in 2015 after the NDNCA workshop with the goal of facilitating communication, collaboration, coordination, and prioritization of nuclear data efforts across multiple program offices, the national laboratories, universities, and industry. The group is composed of nuclear data and applications experts nominated to represent program or national laboratory mission interests. Each interested program office can nominate up to two laboratory researchers to represent their mission interests, to ensure that program-specific needs are communicated, and collaborative opportunities are leveraged. Additionally, each DOE and National Nuclear Security Administration (NNSA) national laboratory is invited to nominate up to two individuals to represent their laboratory's mission and communicate back opportunities.

The NDWG is responsible for organizing the annual WANDA workshops that inform the topic areas called out in the annual NDIAWG FOA. The NDWG solicits input on mission-driven nuclear data needs and suggests topics for the WANDA roadmapping sessions. The workshops are designed to be an open forum to collect recommendations from the larger science community and to document that input. The 
NDWG webpage has recently gone live. It is hosted on the National Nuclear Data Center (NNDC) website and can be found here: https://www.nndc.bnl.gov/ndwg/ [8]. The website contains links to the Nuclear Data Roadmapping and Enhancement Workshop (NDREW), NDNCA, and WANDA workshop reports along with nuclear data needs publications.

The NDIAWG is led by the Office of Science, Office of Nuclear Physics (NP) and is open to all interested federal program managers across DOE, NNSA, and other funding agencies. The NDIAWG communicates regularly on nuclear data needs and planned projects. It releases a cooperative nuclear data FOA each year in order to facilitate co-funding of cross-cutting nuclear data projects. Those offices that cannot participate directly in the FOA can coordinate their funded nuclear data projects with the NDIAWG.

\section{WANDA2020 PROGRAM}

WANDA2020 was held March 3-5, 2020, in Washington, DC. It was comprised of nuclear data needs talks by managers of domestic and international agencies, six roadmapping sessions summarized in Appendix A, a nuclear data pipeline panel intended to inform attendees of how nuclear data are produced, NDIAWG-funded project updates, and a discussion of future nuclear data efforts and potential workshop topics. More than 160 attendees represented national laboratories, universities, and federal programs, and international collaborators and industry representatives were in attendance. The agenda and attendees are listed in Appendix B, and a group photo is provided in Figure 2. Attendees reported finding the workshop valuable because it initiated conversation and presented new opportunities for collaboration among data users, nuclear data experimentalists, and nuclear data evaluators. The link to the workshop presentations is here: https://conferences.lbl.gov/event/292/ [9].

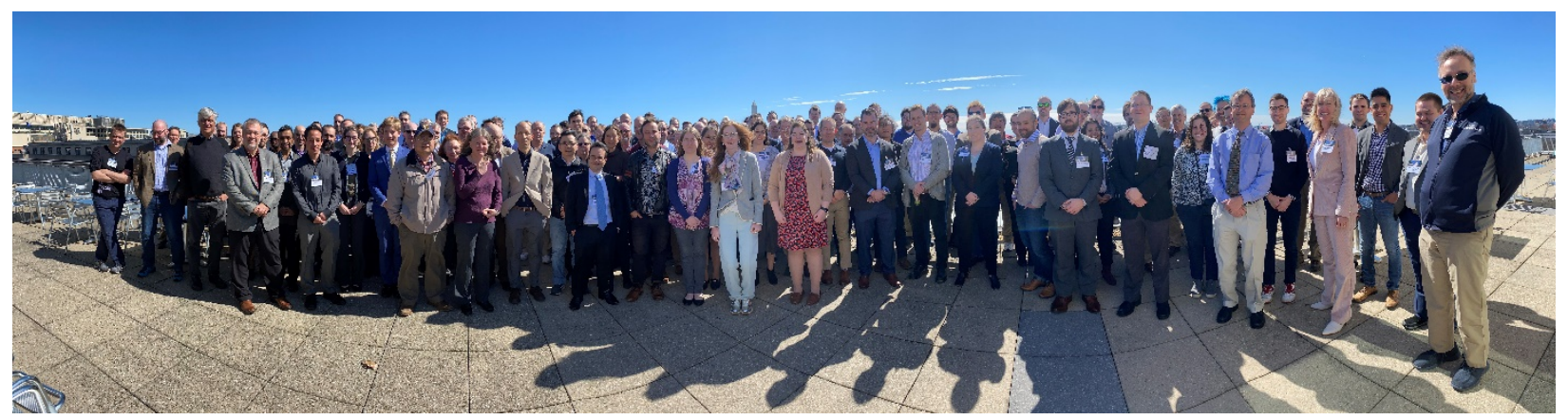

Figure 2. WANDA2020 participants.

\subsection{INTERAGENCY NUCLEAR DATA TALKS}

The welcome talk was given by Tim Hallman, Associate Director for Nuclear Physics. Tim emphasized the need to educate the next generation of nuclear data experts. He announced the new nuclear data application NuRad, which is currently in development, and the many improvements being made to the evaluated nuclear structure data file (ENSDF) database. He stated that there is still a lot of work to be done and that "WANDA is a forum for identifying priorities and setting the stage for important next steps."

The first morning of the workshop included presentations by funding agencies on their specific needs. Not all agencies presented the first morning, and some presented during the topical roadmapping sessions. Below are brief summaries of nuclear data needs described by several agencies: 
Defense Threat Reduction Agency (DTRA): Fission of ${ }^{239} \mathrm{Pu},{ }^{233,235} \mathrm{U}$ prompt neutron and gammas, short-lived fission products, gamma scattering, and nuclear data for detector models.

US Nuclear Regulatory Commission: Cross sections of reactor materials including thermal scattering law, fission yields, decay constants, covariances, and data testing and validation.

Office of Nuclear Energy (NE): For advanced reactors, covariances and data that help predict core reactivity, decay heat, power distribution, feedback response due to material changes during transients, and source term for offsite dose.

Nuclear Criticality Safety Program (NCSP): Capture, total, and thermal scattering cross sections for processing materials and related actinides.

Naval Reactors: Long-lived fission products, elastic scattering for ${ }^{56} \mathrm{Fe}$ and zirconium, ${ }^{236} \mathrm{U}$ neutron capture, cross sections of zirconium and hafnium, irradiation damage, and thermal scattering law.

\subsection{INTERNATIONAL COLLABORATORS}

Arjan Koning, head of the Nuclear Data Section of the International Atomic Energy Agency (IAEA), shared insights on the coordinated research projects (CRPs), an IAEA tool to produce outputs by encouraging collaboration among various parties. He described the new CRP on fission yields, which has broad participation with 16 participants. Additional work included a new International Reactor Dosimetry and Fusion File library that was released in January 2020, a new photonuclear data library, and a new TALYS-based Evaluated Nuclear Data Library (TENDL) [10, 11]. He challenged participants to think about what nuclear data should look like in 2040.

A presentation by Michael Fleming of the Nuclear Energy Agency discussed the Working Party on International Nuclear Data Evaluation Co-operation (WPEC) activities. Recent international projects include Subgroup 40 project CIELO [12], which addressed isotopes ${ }^{235,8} \mathrm{U},{ }^{239} \mathrm{Pu},{ }^{16} \mathrm{O},{ }^{56} \mathrm{Fe},{ }^{1} \mathrm{H}$, and Subgroup 41 on ${ }^{241}$ Am cross sections and Subgroup 42 on thermal scattering law. He also provided updates on the Expert Group on the Recommended Definition of General Nuclear Database Structure (GNDS) and the High Priority Request List. He emphasized the need for development of nuclear data evaluation expertise, the importance of collaboration across borders, and the importance of bolstering the underlying data management infrastructure.

\subsection{THE NUCLEAR DATA PIPELINE}

One of the goals of the WANDA workshops is to educate the broader nuclear community about nuclear data. With this goal in mind, a set of presentations on the major segments of the nuclear data pipeline were given by experts in the field. Figure 3 illustrates the general structure of the pipeline and number of steps and people involved with creating quality nuclear data libraries. Although each segment of the pipeline is interconnected in many ways, the general flow is as shown. 


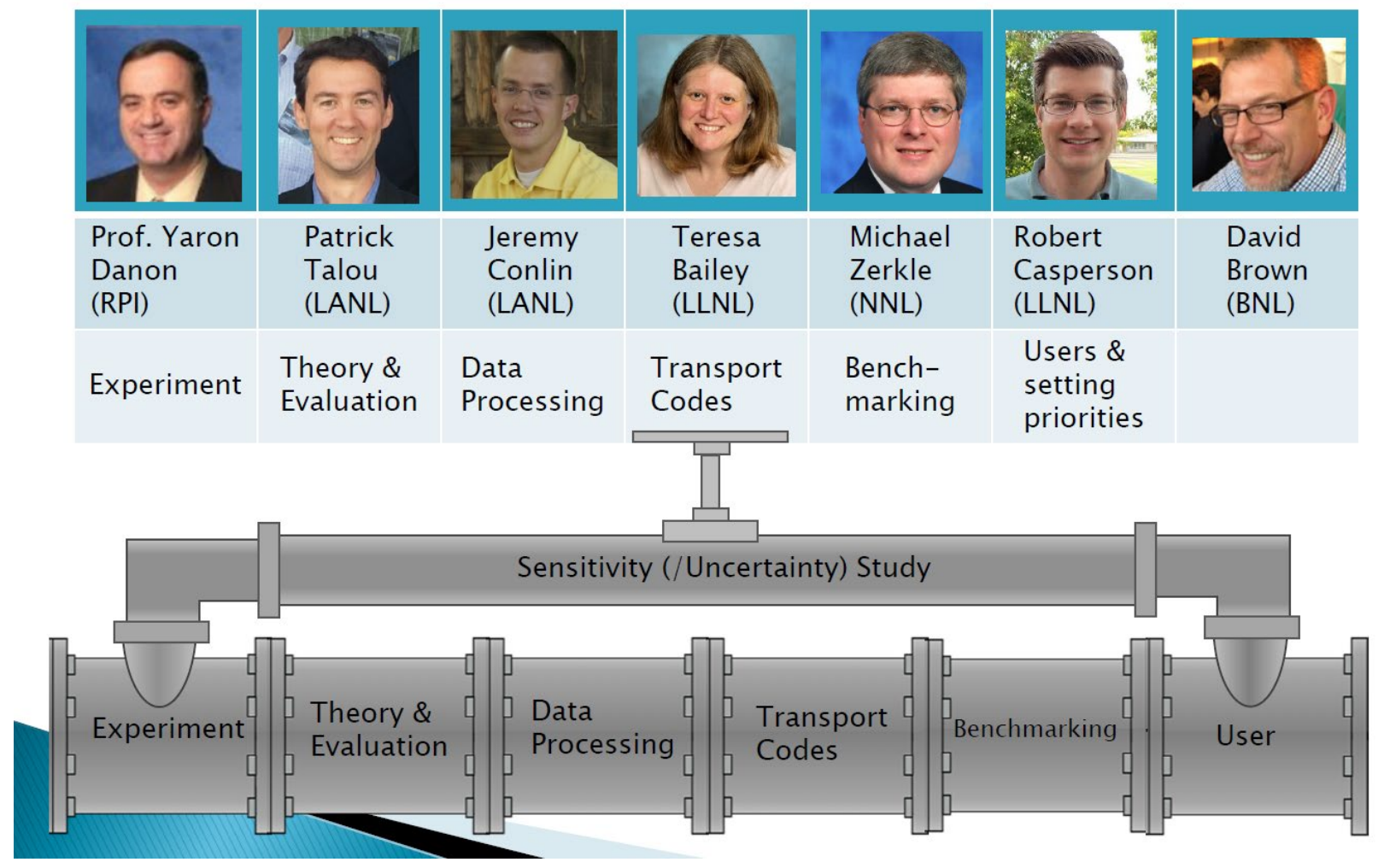

Figure 3. Nuclear data pipeline.

The takeaway messages of the talks included: (1) all segments of the pipeline are essential, (2) communication and cooperation between all segments of the pipeline is necessary, and

(3) uncertainties/covariances must be addressed throughout the pipeline so the impact to applications can be properly assessed. This section includes a summary of each segment talk.

\subsubsection{Experiments}

Low-energy nuclear science (LENS) is relevant to numerous applications such as nuclear power, medical, industrial, defense, and nonproliferation. The current theory in LENS is descriptive, and thus it is not completely predictive and cannot provide the sufficiently small uncertainty required by some applications. Experiments provide the data needed to constrain the evaluation and help reduce and assess uncertainties. To enable a state-of-the-art nuclear data evaluation, a pipeline of targeted, well-quantified, and accurate experiments is required.

Experiments are designed to provide observables that are useful for the application and to constrain the evaluation process. Examples include measurements of neutron transmission and capture yield, fission cross sections and fission products distributions, neutron scattering cross sections, and angular distribution. For neutron-induced reactions, it is also important to measure neutrons, gammas, and other particles emitted from nuclear reactions. To perform such measurements, facilities that include radiation sources and detection systems are needed (e.g., pulsed neutron sources that enable neutron time of flight measurements and monoenergetic neutron sources allow direct measurement of neutron-induced reactions). Monoenergetic and bremsstrahlung photon sources are required to study photon-induced reactions. Other types of facilities enable indirect measurements (e.g., surrogate reactions and inversekinematics) that provide cross sections for isotopes that are hard to obtain or have short half-lives. 
In order to measure accurate nuclear data, infrastructure to perform such measurements must be accompanied by a pipeline of trained and experienced scientists who can move this field forward by developing new, creative measurement techniques and who can be part of the nuclear data pipeline (e.g., via the Cross Section Evaluation Working Group [CSEWG]).

\subsubsection{Theory and Evaluation}

An Evaluated Nuclear Data File (ENDF) provides tabulated data on cross section, multiplicity, emitted particle spectrum, and angular distribution for a particular reaction (e.g., neutrons incident on ${ }^{239} \mathrm{Pu}$ for neutron energies from thermal up to $20 \mathrm{MeV}$ ). The goal is to provide a complete set of evaluated data to the best of our knowledge at the time it is produced. Completeness is a key feature of the data file for it to be of any use in transport codes. While differential experiments (i.e., on specific reaction channels) provide unique and often highly accurate data points, they are always limited in scope (e.g., which reaction channel) and in energy. Theoretical models calibrated to selected and accurate experimental data are developed to fill in the missing parts in the ENDF file. Typical statistical Bayesian techniques are used to fit experimental data and/or tune input parameters entering into the physics model calculations. Covariance matrices representing uncertainties and correlations among nuclear data are also often generated and tabulated by the same token. Selected integral experiments (e.g., well-assessed critical assemblies) can be used to validate and, at times, optimize the underlying evaluated nuclear data within their estimated error bars.

The latest release of the US-evaluated library, ENDF/B-VIII.0, contains more than 550 ENDF files for the neutron sublibrary alone; others include proton, photon, electron-induced and fission yield sublibraries [13]. Nuclear data evaluations are often performed in collaboration with several institutes, including international organizations such as the IAEA, or international partners such as the French Alternative Energies and Atomic Energy Commission, and Japan Atomic Energy Agency. Communication between experimentalists and theoreticians is crucial at the evaluation stage of the pipeline, in particular to perform a correct analysis of the experimental data and their uncertainties, which often dictates the quality of the final evaluated covariance matrices. Additional uncertainties and correlations stem from the theoretical physics models used to complete the evaluated files.

\subsubsection{Data Processing}

Nuclear data processing is the means whereby the evaluated data are made available to the application codes. There are a few primary processing codes in the United States:

- NJOY (Los Alamos National Laboratory [LANL]) [14]

- AMPX (Oak Ridge National Laboratory [ORNL]) [15]

- FUDGE (Lawrence Livermore National Laboratory [LLNL]) [16]

While the details of how each code processes nuclear data are a little different (and unimportant for this discussion), each of the codes read the evaluated data, perform physics calculations, and generate codespecific application data. Some of the physics calculations that are performed include (but are not limited to):

- resonance reconstruction,

- Doppler broadening,

- secondary particle generation,

- KERMA (Kinetic Energy Released in Material) calculation,

- radiation damage cross section generation,

- probability table, 
- $\quad$ self-shield cross sections,

- multigroup collapsing, and

- covariance processing.

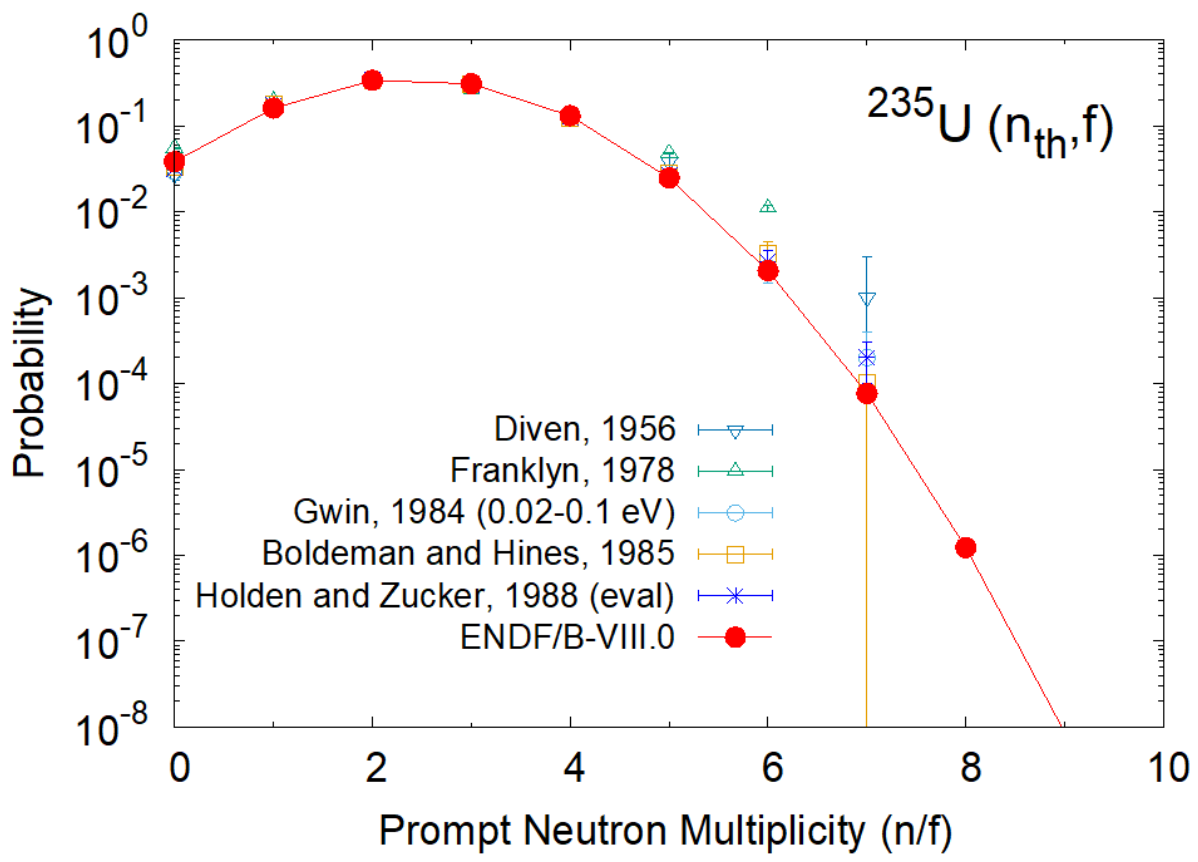

Figure 4. Prompt neutron multiplicity data used for the determination of the ENDF/B-VIII.0 value.

When new data types or formalisms are incorporated into evaluations, updates are required in the format of the evaluated data files. In order to use the data in application codes, updates are also needed in the processing codes, application data formats, and application codes. These updates take time; one should not expect that simply because data are in an evaluation that it is available to end users. An example is perhaps helpful here. Figure 4 shows the prompt neutron multiplicity data introduced into ENDF/BVIII.0. The theoretical work to produce the data and an evaluation was federally funded. However, no funding was made available for data processing or adoption into the application codes. As such, while the data are in the evaluation, processing codes just skip over it, as there is no place to put it into the application data formats and the application codes do not know how to use it.

The processing codes have been developed over many decades and have a lot of capabilities. All processing codes have recently undergone - or are currently undergoing - major renovations to bring them up to modern standards and capabilities. Nuclear data processing is a critical piece of the nuclear data pipeline, without which all the important work done by experimentalists and theorists cannot made available for the end user. This piece of the pipeline is often neglected in the excitement of performing an experiment or developing a new theoretical model. Without ongoing support, these processing codes will be unable to make the new data available to the application codes. 


\subsubsection{Transport Codes}

Transport codes numerically solve the linear Boltzmann Transport Equation using either deterministic or stochastic methods. The calculations are expensive, which drives codes to high performance computing. The transport code developer is often the first customer of nuclear data, and a strong understanding of nuclear data needs is required because the transport code's performance is directly dependent on the quality, accessibility, and availability of the nuclear data.

In some cases, the transport codes rely on lower-fidelity but easier-to-use nuclear data due to historical availability or perceived low impact of higher-fidelity data. An example of this is for neutron-induced secondary particles in fission, capture, or inelastic scatter. Common transport codes such as MCNP [17], SCALE [18], and COG [169] use averages for neutron multiplicity and do not correlate gamma emission, but in certain scenarios, users may benefit from more realistic correlated neutron and gamma multiplicity. The cost of getting capabilities like this to users is not just the cost of measurement and evaluation to make the data available, but the code development and validation activities that must be performed to enable using the data in the transport codes. Efficient delivery of end-user capabilities utilizing new data in transport codes requires regular interaction and communication with transport code developers.

Methods are well established to examine nuclear data uncertainties in codes to support criticality safety analyses, where the key nuclear data uncertainties are in simple cross sections. However, for other types of calculations, such as coupled neutron transport and depletion simulations of reactor systems, additional nuclear data come into play including delayed neutron fractions, fission yields, decay data, branching ratios, and thermal scattering kernels at high temperature. Many institutions are working on covariances for these data and the capability to propagate uncertainty to outputs of interest. In the SCALE system, isotopics uncertainties due to cross section, decay constant, fission yield, and branching ratio can be estimated, but the majority of the covariances are created downstream of ENDF/B.

\subsubsection{Benchmarking}

Benchmarking provides the validation that analytical methods adequately represent reality for a given application. Benchmarks, very well characterized experiments with detailed experimental uncertainties, act as integrated tests of evaluated nuclear data, nuclear data processing codes, and transport codes. Some of the uncertainties in the criticality benchmarks include geometry simplifications, room return, and material impurities. Commonly used benchmarks include critical assemblies, subcritical assemblies, and shielding experiments; they are recorded in the International Handbook of Evaluated Criticality Safety Benchmark Experiments (ICSBEP), International Handbook of Evaluated Reactor Physics Benchmark Experiments (IRPhEP) and the Shielding Integral Benchmark Archive and Database (SINBAD).

A suite of benchmarks is used to validate evaluated nuclear data, provides feedback to the measurement and evaluation community, and identifies required nuclear data improvements. It also provides confidence in well-performing nuclear data. Figure 5 provides an example of a plot of $k_{\text {eff }}$ calculated/experimental values for a test suite of LEU-COMP-THERM (low enriched uranium-composite-thermal spectrum) experiments.

Other types of benchmarks include the Spent Fuel Composition database of measured reactor fuel isotopics. These are required for applications such as nondestructive analysis systems for safeguards and nonproliferation. 


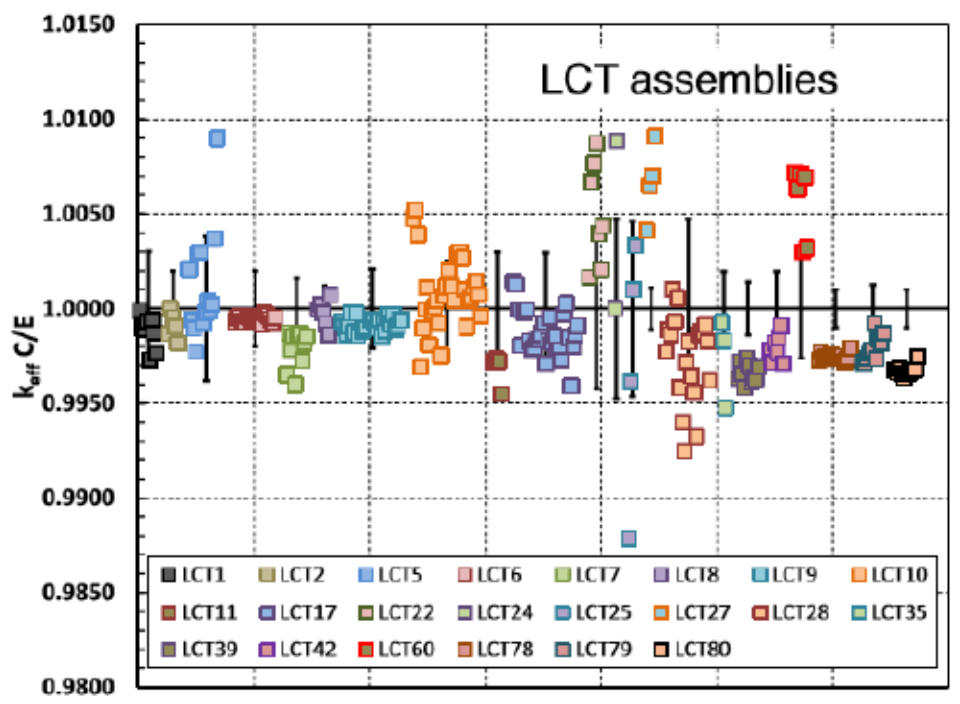

Figure 5. Computed/experimental criticality keff values for a test suite of LEU-COMP-THERM experiments.

\subsubsection{Users, Sensitivity, Uncertainty and Setting Priorities}

Nuclear data users determine the impact of evaluated data on their applications and rely on each step of the pipeline to produce realistic distributions. Some examples of applications include determination of isotopic composition of unknown materials, quantification of neutron leakage in shielding scenarios, and predictions of reactor criticality. Uncertainty quantification in these applications help inform decisionmakers and identify sources of uncertainty that could be reduced. When uncertainties are not available, this assessment cannot be made.

Uncertainty quantification involves propagating input distributions through a model of interest and analyzing the output distributions. These distributions often include correlations between energies and reactions, and the distribution widths are often described with covariance matrices. Uncertainties can be calculated by combining the sensitivity vector of the model with the covariance matrix, or by using Monte Carlo sampling of the input distribution and running ensembles of calculations; the best methodology depends on the problem of interest, and investment in new uncertainty propagation techniques is important. Simple uncertainty propagation can result in contributions that are unacceptably large for some applications, and additional constraints are needed to reduce those contributions. This can be thought of as inverse uncertainty quantification, with the experimental distribution of the constraint propagating backwards through a model to reduce uncertainties in the nuclear data. This process requires relevant integral benchmark data for the application of interest.

Currently, the largest challenge users face in propagating nuclear data covariances is in evaluating the credibility of those covariances. In many cases, covariances are not available, which leaves the user waiting for the isotope to be reevaluated. In the other cases, covariances are poorly evaluated and either produce inaccurate uncertainties or again leave the user waiting for reevaluation. Methods for quantifying the credibility of covariances and filling in missing covariances are a very high priority. Many of the methods for validating covariances rely on credible experimental data, so validating the experimental data are also a high priority. Finally, the impact of proposed experiments on application quantities is not always obvious, and a process for quantifying that impact is needed. 


\section{DISCUSSION SESSION HIGHLIGHTS}

WANDA2020 included six half-day discussion sessions headed by two or more session leads who are experts in the corresponding technical areas and one early career contributor. Each session was supported by a notetaker to capture comments of participants. The goal of the sessions was to obtain unfiltered community input from both users and producers of nuclear data on priority nuclear data needs, the applications impacted, and potential solutions. Participants were asked to consider past and current nuclear data projects within the United States and internationally and the best ways to leverage and avoid conflicts among these efforts.

The summaries below discuss the primary takeaways from each session. The detailed session summaries provided in Appendix A were written by the session leads and reviewed by session participants. Participation in discussion was strong in all sessions, and the intent was to include all viewpoints in the summaries; however, recommended priorities and solutions may not be comprehensive.

\subsection{MACHINE LEARNING AND ARTIFICIAL INTELLIGENCE FOR NUCLEAR DATA}

The goal of this session was to discuss where artificial intelligence/machine learning (AI/ML) have already begun to impact nuclear data research, the ways AI/ML can be leveraged to solve current problems in the nuclear data pipeline, and where the community can focus efforts to maximize long-term impact in the development and application of nuclear data. This is a quickly evolving area that has the potential to transform all corners of nuclear data research and to address critical short-term and long-term needs along the nuclear data pipeline.

\section{Summary of recommendations:}

- Foster collaborations among nuclear data researchers and experts in the AI/ML community.

- Develop surrogate physics models and use them to sequentially search and optimize experimental designs.

- Process complex relationships between nuclear data and integral experiments to develop rigorous validation approaches, and identify systematic trends in nuclear data that were missed by human evaluators.

- Establish validation methods to assess uncertainties.

- Develop AI/ML tools that augment nuclear data expertise, not replace it.

\subsection{DETECTOR MODELS, ATOMIC DATA, AND STOPPING POWERS}

The goal of this session was to focus on nuclear data needs related to the design, development, and interpretation of data from traditional and novel radiation detectors and accelerators for basic science and applications. This includes atomic, nuclear, and optical data for transport modeling, stopping powers, Cerenkov and scintillator light yields, and x-ray emission for detection and cross-cutting application needs.

\section{Summary of recommendations:}

- Evaluate organic scintillator properties, resulting in a curated database.

- Add language to relevant FOAs that instruct respondents to describe how software underlying proposed modeling will be made available to the evaluation and benchmarking community.

- Conduct interlaboratory evaluation of long-lived actinide decay schemes focused on branching ratios, angular correlation, and conversion mixing ratios.

- Conduct scoping study to assess photonuclear data needs for cargo scanning applications. 
- Improve inelastic neutron scatter cross-section and gamma production measurements of $\mathrm{Cu}, \mathrm{Ar}$, $\mathrm{W}, \mathrm{Al}, \mathrm{C}, \mathrm{Fe}$, and other steel constituents.

- Conduct direct measurements of beta energy spectra for high-yield, high-Q-value fission products.

\subsection{COVARIANCE/ SENSITIVITY/ VALIDATION SESSION}

The goal of this session was to examine methods and capabilities to quantify uncertainties and their impact to applications. Nuclear data covariances are needed to assess operational, safety, and economic margins on application quantities simulated with nuclear data. Sensitivity and validation calculations are vital to enable propagating covariances to calculate output quantities and validating that the nuclear data themselves have reasonable predictive power for the application represented by the benchmark. There is a need to address unrealistic and missing covariances, uncertainty/sensitivity tools and methods, and the tuning of nuclear data and covariances for specific applications.

\section{Summary of recommendations:}

- Develop a complete, standardized set of covariance data, including fission product yields, thermal scattering, and angular distributions.

- Verify mathematical constraints and test reliability prior to library release.

- Create enhanced sensitivity/uncertainty tools to propagate covariances to applications.

- Develop nontraditional and application-specific validation experiments.

- Address the fact that libraries are optimized to criticality experiments vs a general-purpose library.

- Conduct further discussions on how to provide missing covariances.

- Improve communications on covariances and validation experiments with the user community.

\subsection{NUCLEAR DATA FOR ISOTOPE PRODUCTION AND NUCLEAR DATA TARGET PRODUCTION CROSS SECTIONS}

The goal of this session was twofold. The first goal was to highlight the progress with nuclear data for isotope production and the infrastructure needs to support future progress in nuclear data. The nuclear data addressed includes charged particle-, neutron-, and gamma-induced reactions. The second goal was to examine the production capabilities and infrastructure needs for both stable and radioactive targets used in nuclear data experiments.

\section{Summary of recommendations:}

- Provide nuclear data to predict the nuclide yields produced by the Facility for Rare Isotope Beams (FRIB) on water for heavy ions up to $200 \mathrm{MeV}$.

- Improve cross sections for reactor production of trans curium isotopes and enhance modeling capabilities to reduce production costs.

- Improve data to support reactions induced by He and Li beams.

- Improve and expand modeling capabilities for charged-particle-induced isotope production.

- Develop expanded capabilities for ultra-thin nuclear data targets, fission sources, and other radioisotope targets in addition to electrodeposition.

- Create a catalogued repository for available target materials. 


\subsection{SCATTERING, TRANSPORT, AND SHIELDING}

The goal of this session was to focus on all shielding applications and scattering reactions over all energy ranges, from thermal to $>10 \mathrm{MeV}$. Discussion included the current state of nuclear data, transport calculations and methods, and opportunities for improvement in nuclear data and evaluations.

\section{Summary of recommendations:}

- Develop well-characterized shielding benchmarks to take full advantage of modern shielding methods to reduce uncertainties in radiation shielding that result in overconservative design.

- Make data available to transport calculations by an application programming interface to provide a common set of data to all codes.

- Establish robust verification and validation in transport codes.

- Conduct quasi-integral nuclear data measurements, which are a new tool for benchmarking/validation; more work is needed for common shield/reflector materials.

- Take advantage of the renaissance in thermal neutron scattering data driven by advances in atomistic simulation-based evaluation methods.

\subsection{NEUTRON-INDUCED GAMMA PRODUCTION AND GAMMA DECAY}

The goal of this session was to explore the role of neutron-induced gamma-ray cross sections and spectra for a wide range of applications and discuss the challenges involved in evaluating this type of data. Contributions were obtained to develop a plan (i.e., roadmap) that best addresses outstanding needs for gamma-ray data from measurement through compilation, modeling/evaluation/validation to dissemination.

\section{Summary of recommendations:}

- Remedy the significant deficiencies in the gamma production nuclear data as needed for applications.

- Repair the inconsistencies between the ENSDF decay library and the ENDF/B reaction library.

- Conduct targeted benchmark measurements, along with code development, to enhance the use of improved data to accurately model neutron-induced gamma emission.

\section{DISCUSSION, CONCLUSIONS, AND NEXT STEPS}

\subsection{ACCOMPLISHMENTS}

Since 2015, when the NDWG was formed, critical avenues of communication have been established between the nuclear data community and funding agencies. The NDWG provided information on the importance of nuclear data to mission success and recommended steps to improve the data. A key element to successful progress is the collaboration of effort among programs. Improving nuclear data are expensive, typically requiring 5-6 years for the data to traverse the full pipeline to the user. For this reason, nuclear data projects have not fit into the typical 3-year funding strategy. Recognition of these facts led to the NDIAWG FOA, which encourages projects that are of longer time frame and co-funding by multiple agencies so that larger projects became financially feasible.

The annual workshops are connecting nuclear data users to the nuclear data community and providing the feedback required to improve nuclear data with mission impact. The nuclear data users are gaining an understanding of nuclear data uncertainties and how the data are produced, and the nuclear data 
community are better understanding the needs. This communication provides a consensus of ideas on nuclear data priorities and the efforts required to improve nuclear data.

The issues identified in the workshops as high-priority and cross-cutting led to the co-funding of targeted projects through the NDIAWG FOA to improve nuclear data. In addition, other agencies have funded nuclear data efforts complimentary to the NDIAWG FOA, and they participate in interagency coordination efforts. These agencies include NE, the DOE Isotope Program, DTRA, and the Department of Homeland Security. Nuclear data projects initiated since the establishment of the NDWG include:

- Fission yield experiments and evaluations including beta decay measurements for reactor antineutrinos

- Inelastic neutron scattering on ${ }^{238} \mathrm{U}$

- $\quad(a, n)$ reaction scoping study

- Neutron-induced gamma production scoping study

- Nuclear data target production method development

- Charged-particle reactions for isotope production

- Nuclear data pipeline infrastructure

- Gamma decay data improvements

Other benefits of the communication established during the workshops include growth of the ${ }^{238} \mathrm{U}$ inelastic scattering project, made possible by additional funding from interested interagency partners, enabling the project to have greater scope and impact with minimal additional costs.

A new WPEC subgroup on "developing an automatically readable, comprehensive and curated experimental reaction database" was initiated and approved due to discussions initiated at WANDA2020, and a CRP called "Updating Fission Yield Data for Applications" was initiated by the IAEA due to discussions at NDREW.

The workshops provide direction for university projects and facilitate collaboration with national laboratories. Use of university facilities can reduce experiment costs while training new nuclear data experts. Many new students are interested in being trained in nuclear data methods to fill the need for additional expertise. Universities who participated in the nuclear data workshops include:

- University of California-Berkeley

- Rensselaer Polytechnic University

- Air Force Institute of Technology

- University of Tennessee-Knoxville

- Oregon State University

- University of Massachusetts, Lowell

- University of Notre Dame

- Central Michigan University

- Michigan State University

- Colorado School of Mines

- Ohio State University

- North Carolina State University

- Duke University

- Texas A\&M University 


\subsection{CONSENSUS PRIORITIES FOR FUTURE WORK}

The overarching goal of WANDA2020 was to identify and prioritize specific challenges for resolution and to discuss the efforts required to get existing nuclear data into US nuclear data libraries and available to the users. Each year, several broad topics come to the forefront as priorities that require action to resolve, or those that require additional exploration.

This year, several broad, cross-cutting priorities that support the nuclear data pipeline were identified: (1) the need for application-specific benchmarks to better quantify the impact of nuclear data uncertainties and the methods to quantify the impact of the nuclear data uncertainties, (2) the need for improved covariances in general and application-specific covariances to minimize safety margins in some instances, and (3) the further examination of ways that AI/ML can support the production of nuclear data across all segments of the nuclear data pipeline.

The ability to sustain the nuclear data workforce is considered critical, and several funded projects require the training of new staff. This topic has been brought up several times, but a more comprehensive examination of potential solutions is required. It was recommended that efforts be made to expand university participation in WANDA2021 and that this topic be addressed during the workshop including student access to mentors, summer courses, and university programs.

\subsection{NEXT STEPS}

Planning for WANDA2021 is under way, as is the development of a new NDIAWG FOA. Based on feedback from attendees, WANDA2021 will maintain the same format in a larger venue due to the increasing year-over-year attendance. There is interest to expand university participation, and the addition of a student poster session is recommended.

The NDWG updated its charter and is expanding its membership to include new federal agency representatives as well as representatives from the national laboratories. National laboratory participation will help inform the NDWG of mission priorities as it plans upcoming efforts. The inclusion of universities is also being considered. Upcoming efforts include examining ways to have a broader reach to educate nuclear data users about the nuclear data pipeline and uncertainties in the data. Further discussions will be held on how the US nuclear data libraries can best meet the needs of all applications, and the effort required to create a US high-priority request list will be examined.

\subsection{REQUEST ADDITIONAL INFORMATION}

Requests for an invitation to WANDA2021, requests for more information, or suggestions for roadmapping session topics can be sent to the corresponding author, Catherine Romano, at romanoce@,ornl.gov.

\section{ACKNOWLEDGMENTS}

The organizers would like to thank Amanda Lewis, Eric Matthews, Morgan Fox, and Pedro Valdez from the University of California-Berkeley Department of Nuclear Engineering for serving as rapporteurs. They would like to thank Julie Marchand and Lisa Felker for their terrific job handling the logistics, and NNSA fellow, Rebecca Lewis, for assistance with organizing the Friday session. They also wish to express their gratitude to Prof. Allison Macfarlane from George Washington University and Ms. Samantha D'Introno from the Nuclear Science and Security Consortium for providing an outstanding venue for the meeting at the Elliot School of International Affairs. 
Lastly, the organizers want to offer a special word of thanks to the pipeline speakers and the tireless session leaders who worked hard to prepare for, run, and write up the breakout sessions. We deeply appreciate their commitment to their programs and applied nuclear science as a whole.

Support for this workshop was provided by the NDIAWG.

The work by staff from several laboratories was performed under the auspices of the DOE at ORNL under contract DE-AC0500OR22725, LANL under contract DE-AC52-06NA25396, Lawrence Livermore National Laboratory under contract DE-AC52-07NA27344, Pacific Northwest National Laboratory under contract DE-AC05-76RLO1830, LBNL under contract DE-AC02-05CH11231, and Brookhaven National Laboratory under contract DE-AC02-98CH10886.

\section{REFERENCES}

1. Bernstein, L. A., D. Brown, A. M. Hurst, J. H. Kelley, F. G. Kondev, E. A. McCutchan, C. D. Nesaraja, R. Slaybaugh, and A. Sonzogni. Nuclear Data Needs and Capabilities for Applications Whitepaper. No. LLNL-CONF-676585. Lawrence Livermore National Lab.(LLNL), Livermore, CA (United States), 2015. https://bang.berkeley.edu/events/ndnca/whitepaper.

2. Romano, Catherine E. The Nuclear Data Working Group: Accomplishments and Future Plans. Oak Ridge National Lab.(ORNL), Oak Ridge, TN (United States), 2017.

3. Nuclear Data Interagency Working Group / Research Program, DOE National laboratory Announcement Number: LAB 17-1763, April 26, 2017. https://science.energy.gov/ /media/grants/pdf/lab-announcements/2017/LAB 17-1763

4. Nuclear Data Interagency Working Group / Research Program, DOE National Laboratory Announcement Number: LAB 18-1903, March 26, 2018. https://science.energy.gov/ /media/grants/pdf/lab-announcements/2018/LAB 18-1903.pdf

5. Nuclear Data Interagency Working Group / Research Program, DOE National laboratory Announcement Number: LAB 19-2114, April 19, 2019. https://science.osti.gov//media/grants/pdf/lab-announcements/2019/LAB 19-2114.pdf

6. Romano, Catherine E., Timothy Ault, Lee Bernstein, Rian Bahran, Bradley T. Rearden, Patrick Talou, Brian Quiter et al. Proceedings of the nuclear data roadmapping and enhancement workshop (NDREW) for nonproliferation. No. ORNL/LTR-2018/510. Oak Ridge National Lab.(ORNL), Oak Ridge, TN (United States), 2018.

7. Bernstein, Lee, Catherine Romano, David A. Brown, R. J. Casperson, Marie-Anne Descalle, Matthew Devlin, Chris Pickett, Brad Rearden, and Cristiaan Vermeuelen. Final report for the workshop for applied nuclear data activities. No. LLNL-PROC-769849. Lawrence Livermore National Lab.(LLNL), Livermore, CA (United States), 2019.

8. NDWG. Assessed June 23, 2020. https://www.nndc.bnl.gov/ndwg/

9. WANDA2020. Assessed June 23, 2020. https://conferences.lbl.gov/event/292/

10. Koning, Arjan, Stephane Hilaire, and Stephane Goriely. "TALYS-1.6 A Nuclear Reaction Program." User Manual, NRG, The Netherlands (2013).

11. Brown, David A., M. B. Chadwick, R. Capote, A. C. Kahler, A. Trkov, M. W. Herman, A. A. Sonzogni et al. "ENDF/B-VIII. 0: The 8th major release of the nuclear reaction data library with CIELO-project cross sections, new standards and thermal scattering data." Nuclear Data Sheets 148 (2018): 1-142. 
12. Koning, A. J., D. Rochman, J-Ch Sublet, N. Dzysiuk, M. Fleming, and S. Van Der Marck. "TENDL: complete nuclear data library for innovative nuclear science and technology." Nuclear Data Sheets 155 (2019): 1-55.

13. D.A. Brown, M.B. Chadwick, R. Capote et al., "ENDF/B-VIII.0: The 8th Major Release of the Nuclear Reaction Data Library with CIELO-project Cross Sections, New Standards and Thermal Scattering Data," Nucl. Data Sheets 148, 1-142 (2018).

14. Macfarlane, Robert, Douglas W. Muir, R. M. Boicourt, Albert Comstock Kahler III, and Jeremy Lloyd Conlin. The NJOY Nuclear Data Processing System, Version 2016. No. LA-UR-17-20093. Los Alamos National Lab.(LANL), Los Alamos, NM (United States), 2017.

15. Wiarda, D., M. E. Dunn, N. M. Greene, M. L. Williams, C. Celik, and L. M. Petrie. "AMPX-6: a Modular Code System for Processing ENDF/B." ORNL/TM-2016/43 (2016).

16. B. R. Beck and C. M. Mattoon. FUDGE: a toolkit for nuclear data management and processing. No. LLNL-PROC-648476. Lawrence Livermore National Lab.(LLNL), Livermore, CA (United States), 2014.

17. C.J. Werner, et al., "MCNP6.2 Release Notes", Los Alamos National Laboratory, report LA-UR-18-20808 (2018).

18. SCALE Code System, ORNL/TM-2005/39, Version 6.2.4 (April 2020)

19. R. M. Buck, E. M. Lent, "COG User's Manual: A Multiparticle Monte CarloTransport Code," Lawrence Livermore National Laboratory, Livermore CA, UCRL-TM-202590, 5th Edition (Sept. 1, 2002) 
APPENDIX A. ROADMAPPING SESSION SUMMARIES 


\section{APPENDIX A. ROADMAPPING SESSION SUMMARIES}

\section{A.1 MACHINE LEARNING AND ARTIFICIAL INTELLIGENCE FOR NUCLEAR DATA}

\section{Session Leaders:}

- Vladimir Sobes, UTK

- Mike Grosskopf, LANL

- Kyle Wendt, LLNL

- Michael Smith, ORNL

- Patrick Talou, LANL

- Dave Brown, BNL

\section{A.1.1 Introduction}

The fast spread and impact of machine learning (ML) and artificial intelligence (AI) models to diverse areas of physical sciences indicate their tremendous potential to address critical issues and potential bottlenecks in the nuclear data pipeline. The nuclear data community has identified a number of key areas in which AI/ML advances already show substantial promise to make significant impacts, both in the short term and long into the future. By leveraging advances made in other areas of science and, simultaneously, driving innovations in $\mathrm{AI} / \mathrm{ML}$, we can address the needs of the community to provide more rapid, accurate, and robust evaluations, quicker compilation of both data and critical contextual information from published experimental work and optimal experimental design for validation. Additionally, AI/ML surrogate models may be used to incorporate more representational and realistic physics models into transport code simulations, as well as rapidly reproduce the results of complex multi-physics codes relevant for a wide range of applications. Targeted investments are needed now to fully realize the potential of $\mathrm{AI} / \mathrm{ML}$ in nuclear data.

A critical portion of this investment should be directed to fostering collaborations between nuclear researchers and experts in the AI/ML community, especially within the Department of Energy labs and universities. Through collaborations, more appropriate algorithms for solving critical research problems may be most efficiently determined and subsequently trained, tuned, and deployed for maximum scientific impact. While open source tools like TensorFlow, Keras, PyTorch, and scikit-learn are valuable because they can be used for rapid exploration of new and innovative ideas, in the hands of non-experts, they can yield biased results with unphysical properties.

There are other areas for investment as well. To enhance the reproducibility of results, and to best leverage advancements across different areas of the pipeline, efforts to collect and share fitted models and data should be explored. By including notes on applicability and limitations of different AI/ML approaches, these collections may be more robust. Another focus area is on developing rigorous approaches for validating the trustworthiness of $\mathrm{AI} / \mathrm{ML}$ methods in the nuclear data context, as these validations will be necessary before these tools can be deployed in certain applications where safety is paramount (e.g., nuclear energy, nuclear security). The integration of uncertainties arising from the use of $\mathrm{AI} / \mathrm{ML}$ tools into the larger uncertainty quantification (UQ) process in nuclear data could be an essential ingredient of future validation efforts. Finally, advances in AI/ML should be considered a method for augmenting nuclear data expertise - to supplement the judgement and work of experts, not replace them. 


\section{A.1.2 Evaluations and Processing}

Modern evaluations are built from a collection of disparate phenomenological models which are fit to experimental data. Ideally, the best possible theoretical models of the underlying structure, reaction and fission physics would be included. However, while capable of capturing and predicting trends across the nuclear chart (i.e., correlations between observables), these theoretical models are computationally intensive and often lack the descriptive power and accuracy needed to reproduce experimental data. The consequence is that evaluation models have limited predictive power, which leads to progressively lowerquality evaluations for unstable or difficult-to-measure nuclei where little or no data exists. Machine learning has the potential to address this issue on multiple fronts. Emulators have already proven their usefulness in providing tremendous speedup of fundamental DFT and QMD calculations for mesoscopic material science and chemistry. Such emulators could be used to address the extreme computational expense and permit the partial inclusion of models into modern evaluations. While present theoretical models could be insufficient for evaluations where a great deal of data exist, AI/ML may significantly enhance extrapolations to regions of the nuclear chart where little or no data exists. Further, theoretical models will necessarily impart more physically correct correlations between observables, both in the sense that correlated observables are better described, and that the extracted covariance matrices better describe the relationships between different processes.

The applications of machine learning continue from the specific nuclear reaction into validating the evaluation libraries in their entirety with respect to integral experiment and into processing those libraries for applications. Insight into the defects and missing important physics can be gained by studying libraries. Tools such as unsupervised learning have the potential to help identify new systematic trends in the nuclear data evaluations that may have been missed by human evaluators and be a critical aid for enhancing or correcting our models and methodology. Even in cases where we cannot correct our models, it opens the possibility to better account for their defects and avoid overfitting. Machine learning may also enhance how we post-process and encode nuclear data for applications. Two areas ripe for study are compressing post-processed libraries into a memory-limited form and building better/more adaptive multigroup cross sections. This is particularly important as high-performance computing centers move toward more processing power with less memory.

\section{A.1.3 Experiments / Compilations}

The compilation and analysis of experimental data are a crucial step in the nuclear data evaluation pipeline. Without useful, accurate, well-documented and vetted experimental data sets, the resulting evaluated files can lead to significantly erroneous and biased results that are further propagated to applications - with possibly disastrous consequences. The EXFOR experimental data library constitutes a unique and valuable resource for the nuclear data community. However, in its current form, it does not yet satisfy all the needs of evaluators, especially concerning automatic reading of large amount of data and ML-supported interpretation thereof, a situation that is exacerbated if modern ML/AI algorithms are to be unleashed on it.

To make large-scale machine learning with reaction data possible, the community will need to develop a new database to store the data sets that have been vetted, standardized, and - in some cases - adjusted. The final version of the data sets in this database should be standardized (in formatting, metadata tags and uncertainties), quality-verified (checked for compilation errors and experimental biases and updated to new standard and structure values), and well-characterized (uncertainties standardized using experimental uncertainty templates). The compilation of data into this new database should be based on communication between the evaluator or qualified data users, the original compilers, and the authors of the published work to ensure that the highest quality database is created. To streamline data vetting, natural language 
processing (NLP) tools need to be developed to simplify the correction of compilation errors, and available data quality verification software should be utilized.

With targeted investments, NLP compilation of nuclear data may become the norm. However, there are many challenges to automated NLP, e.g., processing errors and issues with PDF files. While there have been recent developments with NLP tools for other scientific fields, further development is needed for table and figure processing, for incorporating a lexicon of nuclear data terms and symbols, and for developing an interface for expert validation to leverage automated NLP processing capabilities in nuclear data.

\section{A.1.4 Validation}

Validation of nuclear data are a key pillar in the nuclear data pipeline, and the potential for AI/ML to impact the validation process is significant. AI/ML has the potential to process complex relationships in the large spaces spanned both by nuclear data and simulated integral experiments. It may be invaluable in directing the design of optimal validation experiments to probe the potential deficiencies in ND models and evaluation, utilizing iterative feedback in the estimation process. Additionally, the incorporation of information about the inability to predict observations in benchmarks across applications and utilize this information holistically using $\mathrm{AI} / \mathrm{ML}$ may reduce potentially compensating errors due to adjustment to a particular application. This approach will require the sharing of benchmark data and metadata across application domains in a way that allows relationships between nuclear data and discrepancies to be captured by AI/ML, while respecting potential limitations due to information sharing related to security and intellectual property.

To build toward that goal, advances can be made now in both AI/ML-guided optimal experimental design and ML-guided search for deficiencies in nuclear data estimates with respect to integral experiments. The areas of sequential design/optimization using Gaussian processes and deep neural networks show exciting promise to couple with physics models for searching a wide space of experimental designs. Additionally, advancements in reinforcement learning show tremendous potential in sequential decision-making tasks that may be applicable in this area as well. ML prediction models are well known to capture complex relationships between input features to the model and the target of prediction to obtain impressive accuracies. Utilizing tools for ML interpretability, the relationships learned by the model can be communicated to nuclear data experts to allow for directed investigation into potentially unexpected deficiencies in nuclear data.

Synergistically, the expertise developed over decades for nuclear data validation can also drive developments of approaches to validation of $\mathrm{AI} / \mathrm{ML}$ methods that are desperately needed in this community and beyond. Given the importance of nuclear data in safety and security applications, building trust in the stability and robustness of results obtained through deployment of AI/ML methods is critical. Advancements in interpretability ensures that the way in which the AI/ML results are obtained avoid relying on spurious relationships in the data, while showing results are stable to variations in data, to the ML fitting process, and across implementation in the community will ensure that AI/ML results are trustworthy.

\section{A.1.5 Applications}

Specific applications, such as detector model responses or correlated signatures of nuclear physics processes (e.g., fission) cannot rely solely on average quantities as tabulated in ENDF/GNDS-formatted libraries. Instead, they require the incorporation of complex physics models into transport simulations, significantly enhancing the computational cost of such calculations. Emulators developed from these more fundamental calculations could be developed and render such simulations tractable, opening a new 
frontier for transport code capabilities. Such developments would complement, not replace, the existing tabulated nuclear data libraries.

Another application of interest is the identification of features and hidden patterns in the complex, highly multi-dimensional and correlated phase space of nuclear data, especially as used in the simulation of various nuclear applications, including critical and sub-critical assemblies, pulsed spheres, radiochemistry, reactor designs, and others. ML algorithms associated with well-structured databases of nuclear data and nuclear applications provide an unprecedented opportunity to pull out features and parameters in a comprehensive manner, providing feedback on data evaluations from a wide range of diverse applications. This is an extremely difficult task for individual researchers who are often experts in only pieces of this giant puzzle, and who tend to find practical solutions to problems of limited scope, which can in some cases lead to compensating errors in the nuclear data that negatively impact other applications.

$\mathrm{AI} / \mathrm{ML}$ methods in the development/training of surrogate models also have the potential to contribute to a range of nuclear data applications. First, fast surrogate models have the potential to significantly increase the computational capacity for fast propagation of uncertainties through multi-physics problems. In particular, multi-scale (multi-fidelity) approaches are promising, where several surrogate models, differing in fidelity and speed, can be combined to cover a wide range of simulations. Furthermore, such multi-scale surrogate models can be used to study a very wide design space in the optimization of integral experiments. By combining these surrogates with targeted full-fidelity simulations, the development of on-the-fly learning algorithms may be possible.

\section{A.1.6 Conclusion}

$\mathrm{AI} / \mathrm{ML}$ approaches have tremendous potential to address critical short-term and long-term needs across the nuclear data pipeline. To realize this potential, we have indicated the urgent need for targeted investments to leverage advances made in other areas of science and, simultaneously, drive innovations in AI/ML. Critical areas for investment include: fostering collaborations between nuclear data researchers and experts in the AI/ML community; collecting and sharing fitted models and data along with the relevant notes on applicability and limitations; developing approaches to validate the trustworthiness of AI/ML methods; assessing UQ contributions of these methods; and developing surrogate models to rapidly emulate the results of complex multi-physics codes. Throughout these efforts, the principle should be followed to develop AI/ML tools that augment nuclear data expertise, not replace it, and to ensure that, when necessary, rigorous safety protocols are followed. 


\title{
A.2 DETECTOR MODELS, ATOMIC DATA, AND STOPPING POWERS
}

\author{
Session Leaders: \\ - Bethany Goldblum, UCB \\ - Brian Quiter, LBL \\ - Bruce Pierson, PNNL
}

The WANDA 2020 Session on Detector Models, Atomic Data, and Stopping Powers focused on nuclear data needs related to the design, development, and interpretation of data from traditional and novel radiation detectors and accelerators for basic science and applications. This included atomic, nuclear, and optical data for transport modeling, stopping powers, scintillator light yields, and x-ray emission for targeted and crosscutting applications. The following topical areas were covered: stopping power; signatures, including antineutrinos, neutron and photon-induced signals, $\mathrm{\gamma}$-ray correlations, and x-ray decay branching ratio uncertainties and associated mixing ratios; organic scintillators for fast neutron detection; and evaluated atomic data.

This session was organized into two main segments: programmatic needs and user needs. Program managers from NNSA NA-22, DHS CWMD, and DTRA NT discussed programmatic priorities and data needs for detector modeling. For the NNSA NA-22 near-field portfolio, data needs across a wide range of activities including forensics, safeguards, and proliferation detection were addressed. The DHS CWMD portfolio highlighted photonuclear cross sections, specifically photofission below $10 \mathrm{MeV}$, emphasizing that photofission simulations using MCNP's Cascading Gamma-Ray Multiplicity CGMF tool lack validation. The DTRA NT program indicated prioritization of an understanding of radiation damage effects to enable the accurate modeling of novel detectors materials, particularly improved data and models to aid in understanding of nanomaterials.

The user briefs identified a broad set of atomic, nuclear, and optical data needs. These are addressed in turn below:

\section{A.2.1 Gamma/x-ray Emission}

Improved accuracy and uncertainties in gamma and x-ray decay branching ratios, $\mathrm{x}$-ray fluorescence mechanisms for modeling, $\mathrm{y}$-ray angular correlation function coefficients for modeling, and photon interaction cross sections (which determine photon attenuation coefficients) for select fission products and long-lived actinides are needed. Recent measurements have shown differences in the x-ray decay branching ratios of ${ }^{235} \mathrm{U}$ with implications for material accountancy and safeguards. ${ }^{1}$ These data have immediate applications in safeguards, including improved analytical precision in actinide material assay using densitometry, X-ray fluorescence, and radiation spectroscopy for nuclear forensics, environmental counting, and basic nuclear physics (e.g., fission yield measurements). These data are required for detector modeling and estimation of accurate cascade summing corrections in high-resolution $\mathrm{y}$-ray spectroscopy and $\gamma-\gamma$ coincidence counting. ${ }^{2}$ These data are also needed to enhance analytical precision through close geometric counting, reducing detection limits and increasing sample throughput. The recommended actionable task is a historical and experimental inter-laboratory evaluation of long-lived actinide decay schemes focused on branching ratios, angular correlation, and conversion mixing ratios.

\footnotetext{
${ }^{1}$ M.-C. Lépy, S. Pierre, R. Van Ammel, and M. Marouli, "Photon emission intensities in the decay of U-235," Applied Radiation and Isotopes, vol. 126, pp. 150-153, Aug. 2017, doi: 10.1016/j.apradiso.2016.12.045.

${ }^{2}$ F. Courtine, S. Sanzelle, T. Pilleyre, and D. Miallier, "Calibration of a germanium well-detector using ${ }^{60} \mathrm{Co}$ : The effects of the angular correlation of the two gamma rays emitted in cascade, quantified with Monte Carlo simulations," Radiation Measurements, vol. 61, pp. 78-82, Feb. 2014, doi: 10.1016/j.radmeas.2013.11.007.
} 


\section{A.2.2 Photonuclear reactions}

Photofission data are needed to simulate and interpret experimental signal and background data for active interrogation cargo screening techniques, specifically ( $\mathrm{y}, \mathrm{f})$ reactions induced by photons below $10 \mathrm{MeV}$ in actinides as well as the associated signals following photofission (i.e., prompt and delayed neutron and gamma emission). The $(\mathrm{y}, \mathrm{n})$ cross sections for actinides and structural materials for incident photons below $10 \mathrm{MeV}$ were also identified as important for understanding relevant sources of background. Participants emphasized specific focus on common low-Z materials and typical shielding materials. The International Atomic Energy Agency recently announced updates to their photonuclear library. ${ }^{3}$ At this time and during workshop discussions, it was unclear how these updates impact capabilities to model photofission systems. DHS CWMD indicated a pending Funding Opportunity Announcement (FOA) to measure $(\mathrm{y}, \mathrm{f})$ reactions for major actinides, which has since been released. ${ }^{4}$ A scoping study is further recommended to assess photonuclear data needs for cargo scanning applications.

\section{A.2.3 Inelastic neutron scattering reactions}

Inelastic neutron scattering and the associated gamma production cross sections and spectra are desired to understand a wide range of measurements and detector systems that span a variety of applications. In safeguards and nuclear nonproliferation, these include active neutron interrogation, radioactive material imaging, neutron-induced signatures, cargo scanning, and neutron detection. Improving the fidelity and breadth of inelastic neutron scattering data are a crosscutting need that was identified in multiple sessions at WANDA 2020. As current data and modeling capabilities are lacking, a measurement campaign is recommended. Species of particular interest include $\mathrm{Cu}, \mathrm{Ar}, \mathrm{W}, \mathrm{Al}, \mathrm{C}, \mathrm{Fe}$, and other steel constituents.

\section{A.2.4 Heavy ion stopping}

Stopping power data are important for direct fission cross section measurements and nuclear physics measurements employing beam degraders. ${ }^{5,6}$ Deficiencies in stopping power data related to issues such as effective charge, low velocity stopping, and molecular effects are broadly impactful, but of particular consequence for fission fragment stopping. The nuclear data user community has generally relied on the SRIM (Stopping and Range of Ions in Matter) and TRIM (TRansport of Ions in Matter) codes to provide stopping power and ion range information. The SRIM code uses empirical corrections to theoretical

\footnotetext{
${ }^{3}$ T. Kawano, Y.S. Cho, P. Dimitriou, D. Filipescu, N. Iwamoto, V. Plujko, X. Tao, H. Utsunomiya, V. Varlamov, R. $\mathrm{Xu}$, R. Capote, I. Gheorghe, O. Gorbachenko, Y.L. Jin, T. Renstrøm, M. Sin, K. Stopani, Y. Tian, G.M. Tveten, J.M. Wang, T. Belgya, R. Firestone, S. Goriely, J. Kopecky, M. Krtička, R. Schwengner, S. Siem, M. Wiedeking, "IAEA Photonuclear Data Library 2019," Nuclear Data Sheets, Volume 163, 2020, pp 109-162, doi: 10.1016/j.nds.2019.12.002.

${ }^{4}$ General Services Administration, Department of Homeland Security, Topic No. 4 Solicitation: 70RWMD20R00000005: "Photofission Nuclear Data for Active Interrogation," https://beta.sam.gov/opp/75d27c7bd9db4cf39fd60578c69a7057/view

${ }^{5} \mathrm{P}$. Baldez et al., "Measurements of ${ }^{252} \mathrm{Cf}$ fission product energy loss through thin silicon nitride and carbon foils, and comparison with SRIM-2013 and MCNP6.2 simulations," Nuclear Instruments and Methods in Physics

Research Section B: Beam Interactions with Materials and Atoms, vol. 456, pp. 142-147, Oct. 2019, doi: 10.1016/j.nimb.2019.06.027.

${ }^{6}$ J. T. Morrell, A. S. Voyles, M. S. Basunia, J. C. Batchelder, E. F. Matthews, and L. A. Bernstein, "Measurement of ${ }^{139} \mathrm{La}(\mathrm{p}, \mathrm{x})$ cross sections from 35-60 MeV by stacked-target activation," Eur. Phys. J. A, vol. 56, no. 1, p. 13, Jan. 2020, doi: 10.1140/epja/s10050-019-00010-0.
} 
predictions of model ion stopping. ${ }^{7}$ Discrepancies have been noted between predictions and observed low velocity, high charge ions (i.e., fission fragments) and for stacked target measurements. ${ }^{8}$ While these discrepancies may be due in part to changes in effective charge, models that implement corrections for low velocity still exhibit disagreement with experimental data of 7-30\%. These inconsistencies convey the need for broad and open assessment of the reliability of heavy ion stopping power compilations. Stopping power measurements in specific materials may not be useful to the broader community without improved theory. Thus, a stopping power evaluation is recommended, including experimental data compilation and theory development. A scoping study to assess the types of experiments and sensitivities needed to solve the stopping power data deficiency for relevant ions was identified as the most impactful initial step toward achieving a more comprehensive evaluation.

\section{A.2.5 Antineutrino physics}

To address the reactor antineutrino anomaly and foster the development of applied antineutrino physics technologies, new nuclear physics measurements, neutrino-centered nuclear data infrastructure, and advances in modeling and simulation for antineutrino sources are required. For nuclear physics measurements, improved accuracy and uncertainty are needed in beta energy spectrum shape functions, beta decay level feeding, fission product yields, and relevant covariance data for short-lived, high Q-value fission products. ${ }^{9}$ Simultaneous effort is needed towards integrating diverse neutrino datasets and models into a common standardized format and repository with provisions for public access. In addition to basic nuclear physics inquiry, these data provide benefits for broadening the scope of the validation stage of the nuclear data pipeline (i.e., as an "integral benchmark" for illuminating errors in nuclear data measurements and processing $)^{10}$ and for developing capabilities for remote measurement of fissile material inventory in nuclear reactor monitoring applications. Recommended actionable tasks include direct measurements of beta energy spectra for high-yield, high Q-value fission products, related neutrino modeling to assess impacts of new datasets, and development of standardized nuclear data products for existing reactor antineutrino data.

\section{A.2.6 Neutron response of organic scintillators}

Organic scintillators are useful for fast neutron detection in a wide variety of applications, including basic nuclear physics measurements, safeguards, proliferation detection, and emergency response. Yet, no database of organic scintillator properties (e.g., electron light yield, proton light yield, temporal response) exists nor are these properties sufficiently described by theory to draw inferences from discrepancies between measurements and modeled detector response. Current ionization quenching models, such as the canonical Birks relation, demonstrate discrepancies at low recoil energies and disagreement between particle types. ${ }^{11,12}$ An understanding of quenching phenomena in organic scintillators is a crosscutting

\footnotetext{
${ }^{7}$ James F. Ziegler, M.D. Ziegler, J.P. Biersack, "SRIM: The stopping and range of ions in matter" NIM B Vol 268, Issues 11-12, 2010, Pages 1818-1823, 10.1016/j.nimb.2010.02.091.

${ }^{8}$ P. Sigmund and A. Schinner, "Progress in understanding heavy-ion stopping," Nuclear Instruments and Methods in Physics Research Section B: Beam Interactions with Materials and Atoms, vol. 382, pp. 15-25, Sep. 2016, doi: 10.1016/j.nimb.2015.12.041.

${ }^{9}$ L. McCutchan, "Following Fission Fragments: Nuclear Data for New Neutrino Physics” WBNL Streaming Video, https://www.bnl.gov/video/index.php?kw=neutrinos.

${ }^{10}$ A.A. Sonzogni, et al., "Effects of Fission Yield Data in the Calculation of Antineutrino Spectra for

${ }^{235} \mathrm{U}(\mathrm{n}$,fission) at Thermal and Fast Neutron Energies," Phys. Rev. Lett. 116, 132502 (2016). https://link.aps.org/doi/10.1103/PhysRevLett.116.132502

${ }^{11}$ M.A. Norsworthy, et al. "Evaluation of neutron light output response functions in EJ-309 organic scintillators," Nucl. Instrum. Meth. A. 842, 11 (2017), 20-27. https://doi-org.libproxy.berkeley.edu/10.1016/j.nima.2016.10.035
} 
need, as these data are required for accurate modeling of organic scintillator detection systems and for the interpretation of data from these systems in some cases. ${ }^{13}$ Recommended action is an evaluation of organic scintillator properties, including compilation of experimental data on common commercial crystal, liquid, and plastic organic scintillators and a template for evaluation of these measurements, resulting in a curated database for the basic and applied physics communities.

\section{A.2.7 Experiment and modeling integration}

Previous FOAs have encouraged collaboration within the nuclear data community, particularly between experimentalists and evaluators. Current efforts are actively generating benchmarks and test datasets for associated nuclear data codes. An example is a detector response model database, leveraging the proposed organic scintillator properties database and response model validation (e.g., via the Detector Response Function toolkit, DRiFT). Other suggestions include encouraging staff to release open-source models as part of future publications for use by evaluators and in community validation. Recommended actions are a continued emphasis on integration of experimental studies with the modeling community; particularly, FOAs could ask "Describe how proposed work that includes a modeling component will provide the software underlying the modeling (e.g., MCNP input files) to the evaluation and benchmarking community." In addition, FOAs could encourage inclusion of points-of-contact on the development teams of detector modeling codes as part of future efforts. Establishment of an experiment and modeling working group within the nuclear data community would facilitate such connections.

12 J.B. Birks, The Theory and Practice of Scintillation Counting, Pergamon, 1964, pp. 185-234. https://doi.org/10.1016/B978-0-08-010472-0.50011-2.

${ }^{13}$ J.J. Manfredi, et al., "Proton Light Yield of Fast Plastic Scintillators for Neutron Imaging," IEEE Trans. Nucl. Sci. 67 (2020), 434-442. https://doi.org/10.1109/TNS.2019.2959979 


\title{
A.3 COVARIANCE/ SENSITIVITY/VALIDATION SESSION
}

\author{
Session Leaders: \\ - Denise Neudecker, LANL \\ - Robert Casperson, LLNL \\ - Rike Bostelmann, ORNL \\ - Fredrik Tovesson, ANL
}

\section{A.3.1 Introduction:}

The goal of the Covariance/ Sensitivity/ Uncertainty/Validation session was to identify issues in (a) nuclear data covariances, (b) their propagation to application margins, and (c) nuclear data validation that critically impact application simulations of interest to several programs.

Nuclear data covariances provide an estimate of the uncertainty of their associated nuclear data mean values along with correlation information on the linear dependence between these mean values. These nuclear covariances can be propagated through models of interest such that margins on nuclear data application quantities (e.g., Impact of Xe concentration on reactivity of a reactor system) due to these covariances can be assessed. Usually, sensitivity tools, in conjunction with assumptions on the underlying probability distribution functions, are needed to propagate the covariance data via sensitivity coefficients to uncertainties in output quantities. Sensitivity coefficients describe by how much a simulated application quantity changes due to a change in the underlying nuclear data. In cases where these sensitivity methods based on finite-order derivatives break down in the face of nonlinearities in the applications being studied, sampling-based propagation methods can be used to accurately understand the impact of the nuclear-data uncertainties on application quantities. These quantities are often represented on a smaller scale by validation experiments. Mean values of nuclear data libraries are typically derived from differential measurements and nuclear models. They are frequently tested by simulating validation experiments and comparing the simulated value to their measured values in order to ensure proper performance of the library with respect to applications represented by these integral validation experiments. The term "benchmark" experiment also often appears in reference to validation experiments; these are experiments that have undergone a stringent quality review process by a larger community. One frequently used example is the ICSBEP handbook of criticality benchmarks that includes benchmark descriptions along with evaluated effective multiplication factors, $k_{\text {eff }}$, for experiments with various fissile materials (physical form and composition), enrichments and spectral conditions.

It should be emphasized that good-quality nuclear-data covariances and validation experiments are vital to reliably assess economic, safety and performance margins of applications relying on simulations. No matter how sophisticated the model of a nuclear system, or how detailed the calculation procedures used to analyze them, the results are unreliable if the nuclear-data mean values and covariances entering the simulations are not well-characterized because of the lack of validation experiments or due to improper uncertainty quantification. Equally important, these issues have a profound influence on the ability to provide reliable feedback to evaluators, experimentalists and theoreticians who generate nuclear data on the latter's quality and reliability. This feedback is needed so that these scientists can undertake future research in a more targeted and informed manner and with greater fidelity and cost effectiveness. Furthermore, the importance of sensitivities and uncertainty propagation tools cannot be overstated, as they allow us to bind together nuclear data covariances and application bounds. Without sensitivities and the associated analysis, assigning bounds on application simulations due to nuclear data covariances can become intractable or extremely computationally expensive. 
From the discussion above it should be clear that covariances should appear in and are needed for every part of the nuclear data pipeline. Covariances estimated for differential experimental data in the EXFOR database (openly available database of more than 23000 experiments used in evaluations) and in the nuclear theory enter nuclear data evaluations, and both can contribute to evaluated nuclear data covariances. Evaluated Covariances are then processed along with their mean values and their impact can be assessed on simulations of nuclear data validation experiments and application quantities by means of sensitivity profiles and uncertainty propagation.

The section here will condense the main points of the Covariance/ Sensitivity/ Uncertainty/ Validation session that either arose from the presentations or discussions by summarizing the session agenda, highlighting critical needs and issues, and then presenting a tentative path forward.

\section{A.3.2 Session agenda:}

The session started out with an introduction where D. Neudecker provided the necessary nomenclature and highlighted again that issues in any part of the nuclear data (covariance) pipeline can adversely impact applications. A. Sonzogni gave an example where funding through the Nuclear Data Interagency Working Group Funding Opportunity Announcement (FOA) funding enabled an impact study of incorporating the nuclear data covariances in the analysis of a particular application; the FOA funding for fission product yields triggered research demonstrating that the consideration of correlations between uncertainties of independent fission yields changes antineutrino yield uncertainties in a reactor for ${ }^{235,238} \mathrm{U}$ and ${ }^{235,240} \mathrm{Pu}$ by up to a factor of 1.5 .

The second part of the session highlighted open issues related to nuclear data covariances and validation. D. Neudecker introduced the $\mathrm{CSEWG}^{14}$-covariance-session effort to provide templates of uncertainties expected in measurements. These templates (a) lay out for experimentalists what information (specifically sources of uncertainty) should be provided for a new measurement to maximally impact evaluations, and (b) give evaluators the means to estimate obviously missing uncertainties in old data sets. The latter part addresses the issue that experimental uncertainties are often incompletely captured within EXFOR and the literature and that this can affect the evaluation, potentially leading to unreliable experimental covariances being used within the evaluation process. T. Bailey highlighted the need for complete covariance libraries in order to allow for comprehensive assessments of their impact on simulated application margins, while K. Parsons' talk highlighted the need for realistic scattering-angular distribution covariances. B. Rearden focused on the need for nuclear-data mean values to be more rigorously validated with respect to validation experiments beyond criticality (e.g., total power distribution, radiation damage and temperature of a reactor, burn-up as a function of position, excess reactivity at start-up, control-rod worth for shutdown); this was neglected for ENDF/B-VIII.0, and, therefore, there are obvious mistakes in the nuclear data with respect to specific validation experiments rendering the libraries less useful for applications represented by these validation metrics.

The third part of the session focused on missing tools in the nuclear data pipeline that allow assessment of the impact of covariances on simulated application bounds. K. Wendt presented a machine learning guided approach that can generate missing covariances from information in EXFOR; a similar tool could be used to test whether evaluated covariances are realistic given information in EXFOR. B.J. Marshall pointed out that there are currently no automated tools to do the aforementioned testing; although, e.g., the ORNL code system AMPX, is one code used for ENDF/B processing, and it does a large part of

\footnotetext{
${ }^{2}$ CSEWG stands for "Cross Section Evaluation Working Group". This is a collaborative effort by national
} laboratories, industry and universities to produce, validate and disseminate ENDF/B nuclear data libraries. 
needed automatic verification of mathematical properties of covariances. M. Rising highlighted that direct and efficient uncertainty propagation to applications beyond criticality and feedback to evaluations is often still missing because of missing sensitivity tools.

The last part of the session focused on adjustment of nuclear data and their covariances to specific applications. F. Bostelmann highlighted that nuclear data mean values are often tweaked to reproduce ICSBEP criticality benchmarks while covariances remain unchanged leading to a seeming overestimation of nuclear-data uncertainties with respect to the represented application space. B.J. Marshall discussed existing tools that enable mitigating this issue for criticality experiments by formal adjustment while at the same time emphasizing the needs for a more general adjustment tool going beyond criticality experiments. V. Sobes connected to this talk by emphasizing the need for more diverse classes of validation experiments going beyond criticality.

\section{A.3.3 Identified Needs and Issues:}

Below the needs and issues are summarized according to their broad subject area within the nuclear data pipeline.

\section{A.3.3.1 Differential experimental data covariance needs:}

1. A vetted and more accessible EXFOR is needed in order to provide the best possible input for nuclear data evaluations and to enable comprehensive testing of whether evaluated uncertainties are realistic. One part of this was the request-it also appeared in the Machine Learning/ Artificial Intelligence (ML/AI) session-to generate an EXFOR format that is more easily readable by automatic tools. Right now, raw EXFOR files can be easily interpreted by nuclear-data experts when looking at each of them individually. However, some information (especially related to uncertainties) is so ambiguously formatted such that an automated algorithm cannot meaningfully process this information. However, it is vital that this information be read automatically in order to allow for large-scale testing of whether or not, given differential experimental information, evaluated uncertainties are realistic. Another part of the discussion focused on the need to have more realistic and complete uncertainty information in EXFOR. Templates of expected measurement uncertainties can aid in completing EXFOR information for old data sets for evaluation purposes, but to start, new data sets should be reported with reliable and complete uncertainties. Last but not least, a request was made to store expert knowledge on existing experimental data sets in a central repository to retain this knowledge for future evaluations. The IAEA-Nuclear Data Section (NDS) has developed the EXFOR data correction systems that already allows to correct data by users which would be a starting point for this central repository.

2. A discussion point from previous meetings in this series was re-iterated, namely, that the impact of a new differential data set on nuclear data evaluations and conversely on application simulations should be tested before the experiment is executed, namely during the design phase of this new measurement. A sensitivity study should enable the best possible differential experiment for application needs.

\section{A.3.3.2 Evaluated covariances needs:}

1. Many cross-section covariances are either missing or assigned unrealistic values. It was emphasized that missing covariances are effectively skewing judgments on which nuclear data should be studied by improved theory, additional differential experiments and new evaluations to minimize related 
application uncertainties. If there are no covariances for a quantity, it is impossible to quantify the impact of its uncertainty on a performance/ safety/ etc. metrics for a given application and whether future studies are needed to better characterize this quantity in order to better address a performance metric for a given application. Hence, many session participants agreed in the discussion that a complete covariance library has the highest priority, followed in importance by testing whether the uncertainties are realistic given uncertainties in the models and experimental data used for evaluation.

2. The need for a complete set of fission product yields, thermal scattering and angular distribution covariances was highlighted several times throughout the discussion for various applications.

3. It was agreed that covariance should be verified (i.e., checks of mathematical constraints) and tested to determine its reliability before the release of nuclear libraries as users should not have to be the ones to correct covariance data. To this end, automated tools in addition to existing ones (e.g., AMPX, ADVANCE) are needed to process and correct large amounts of data along with a process in place to use this information by evaluators.

\section{A.3.3.3 Propagation to applications/ validation:}

1. A disconnect between nuclear data producers (including evaluation and validation communities) and users at both the national laboratories and industry came to light. For instance, ENDF/B-VIII.0 nuclear data were not tested with all metrics important for nuclear energy applications rendering specific nuclear data less usable for some of these applications.

2. It was emphasized during the session that (e.g., sensitivity) tools are missing to propagate covariances to various application-relevant responses. Hence, even if complete nuclear data covariance libraries are available, impact assessment on application bounds might be still infeasible because of a missing link in the pipeline. In the case of angular distributions covariances, processing tools are currently not able to process all formats used for ENDF/B-VIII.0, while neither formats nor processing tools exist for thermal-scattering-law covariances.

3. A clear need for non-traditional validation experiments applicable for several application areas, also came also out of this session. Nuclear data evaluations are mostly validated with respect to critical assemblies of ICSBEP. Potential deficiencies in nuclear data that severely impact some applications may be overlooked by constraining nuclear-data validation exclusively to using ICSBEP criticality integral benchmarks. Therefore, more diverse validation experiments are needed including their uncertainties. As an example, temperature-dependent benchmarks were mentioned.

\section{A.3.3.4 Adjustment:}

1. ENDF/B-VIII.0 mean values are tweaked such that they reproduce ICSBEP critical assemblies well, but their covariances remained unchanged. If these mean values and covariances are used for criticality application simulations represented by ICBSEP benchmarks, application bounds due to nuclear data covariances will be distinctly overestimated because not all the data correlations that were used to derive the mean values were captured within the nuclear-data evaluations. These overestimated uncertainties with respect to some integral metrics may lead to unnecessarily large safety margins in some applications represented by criticality experiments, specifically in reactor analysis, while the nuclear-data mean values might be incorrectly skewed for other application areas, thus, increasing bias in nuclear data. Each mean value adjustment should be well documented so that users do not employ ENDF/B-VIII.0 evaluations blindly. Further discussions with nuclear-data experts and users are required to agree upon established methods, guidelines and tools for nucleardata adjustment.

2. No general-purpose tools currently exist for adjusting whole nuclear data libraries for various applications. Some tools exist (SCALE, Whisper) that allow adjustment only with respect to ICSBEP 
criticality values. These tools will likely be strongly linked to the effort to develop sensitivity tools and derivative-free optimization methods (for application quantities where linearity with respect to nuclear data fails) for assessing impact of nuclear data covariances on various integral responses

\section{A.3.4 Suggested Path forward:}

\section{A.3.4.1 Addressing differential experimental data covariance needs}

1. Take steps towards a more automatically readable EXFOR (including all features and uncertainties), starting with the proposal of an international WPEC subgroup on this topic. If this sub-group is approved and successful, the resulting improved EXFOR would form the basis for generating more reliable nuclear-data mean values and covariances which would allow for automated testing of evaluated covariances and the determination whether or not they were realistic.

2. It is recommended that sensitivity studies be undertaken before conducting a nuclear-data experiment in order to determine how to design the experiment so as to maximally impact the target evaluation or application. To this end, the impact of this potential new measurement, including expected uncertainties, ought to be studied on the evaluation and application simulations. This process will likely involve experimentalists, evaluators and nuclear-data users as well as require sensitivity tools for uncertainty quantification. This step should ensure that experiments are maximally impactful for nuclear data evaluation and applications.

3. It is highly recommended that detailed experimental uncertainties are reported along with data to the National Nuclear Data Center and documented in journal publications. This will ensure that the experimental data are maximally useful for evaluations. These uncertainty estimates should be made easily accessible within EXFOR.

\section{A.3.4.2 Evaluated covariances needs}

1. A high-priority covariance list should be established to catch users' needs and to support the discovery of potentially unrealistic covariances.

2. Findings concerning the quality of specific covariances should also be stored in a central repository available to all users and evaluators. This repository could also capture recommendations on how more complete covariance libraries can be formed from existing ones.

3. Tools and algorithms should be developed and extended to provide mid-fidelity covariance evaluations with the specific aim of supplying currently missing covariances. Machine learning tools at LLNL using EXFOR, the past low-fidelity covariance project and TENDL are potential starting points.

4. Automated tools that verify, and correct if necessary, mathematical properties of covariances and test how realistic these uncertainties are given the differential information used for the evaluation should be extended/ developed and made available for broad testing of new libraries.

5. The timeline of the next U.S. nuclear data library must provide ample opportunity to test covariances along with a well-defined process how this information can be disseminated to evaluators for corrections on a short time-scale. The aim is that issues in covariances should be identified and addressed foremost in this beta-phase rather than by users after its formal release. 


\section{A.3.4.3 Propagation to applications/ validation:}

1. A working group should be established as part of CSEWG that encompasses evaluators, validators and a large user community across industry and the national laboratories. The aim of this working group is:

a. To foster better communication with industry and national laboratory users regarding their needs on nuclear data and covariances and how they should be used;

b. To establish a list of integral experimental responses that are recommended to be used for nuclear data validation with the specific aim to make new ENDF/B libraries more applicable to an expanded set of application areas; and

c. To exchange nuclear data validation experiments between users and evaluators.

2. Sensitivity, non-linear uncertainty propagation (e.g., using polynomial chaos expansion or global variance methods) and processing tools should be developed to propagate covariances for all possible nuclear data observables to various relevant integral experiment responses. These tools should allow for impact assessment of nuclear data uncertainties on responses beyond just criticality of benchmark assemblies. Special attention should be given to develop fast tools.

3. The need for more diverse benchmarks should be addressed through a two-pronged effort:

a. Existing databases (e.g., SINBAD, IRPhEP, RPI semi-integral experiments) should be vetted for additional validation experiments and the input of the evaluator-user working group should be leveraged to generate a comprehensive list of integral responses to be used for nuclear data validation of new libraries.

b. New integral experiments should be proposed with the specific aim of going beyond criticality, while allowing for validation of nuclear data in a form applicable to many programs. Special care should be taken to enable disentangling of compensating effects between nuclear data introduced by validating with criticality experiments only. Examples are pulsed-sphere neutron-leakage spectra, sub-critical assemblies, reaction rates, RPI semiintegral experiments, etc.

\section{A.3.4.4 Adjustment:}

1. A general-purpose library, based exclusively on differential data and model calculations, is needed as necessary starting point of any adjustment. Given that there are tweaks of mean values in ENDF/BVIII.0 to better correspond to criticality in ICBSEP benchmarks, the following must be documented:

a. Which experiments were used for the tweaking process of ENDF/B mean values?

b. What validation responses (e.g., ICSBEP benchmarks) were used for tweaking?

c. Were the covariances adjusted in the same process? If not, what is the recommended procedure to adjust the covariances for users' uncertainty studies?

d. All this documentation should be readily available to users in a central repository and should be communicated as part of the evaluator-user working group.

2. General tools that allow for adjusting or augmenting nuclear data libraries with respect to validation experiments relevant for various application areas should be developed, potentially building upon tools that already exist for adjustment to criticality. 


\section{A.4 NUCLEAR DATA FOR ISOTOPE PRODUCTION AND NUCLEAR DATA TARGET PRODUCTION CROSS SECTIONS}

\section{Session Leaders:}

- Etienne Vermeulen, LANL

- Greg Severin, FRIB, MSU

- Ellen O’Brien, LANL

The Nuclear Data for Isotope Production and Target Fabrication roadmapping session was focused on two topics: 1) nuclear data capabilities and needs to support isotope production, and 2) the current target fabrication infrastructure, capabilities, and needs to support nuclear data and other research experiments. Input was gained from both producers and users of nuclear data associated with isotope production.

\section{A.4.1 Session Highlights}

The session began with both the DOE IP and the IAEA discussing their program plans and accomplishments.

\section{A.4.1.1 Department of Energy Isotope Program (DOE IP)}

Within the DOE Isotope Program, cross section needs for isotope production are extensive and the overall goal is to improve data for established isotopes, develop excitation functions for emerging ones, and ensure that the gaps in the current body of available data are addressed. Some priorities that have been identified are: charged particle production, photonuclear interactions, heavy ion interactions, activation of converter materials, fast neutron-induced reactions, and certain reactor neutron-induced reactions for heavy isotope production. There is an extensive list to improve, update, and expand current databases.

From the program side, efforts are currently funded for $\mathrm{Np}-236$ as well as photonuclear reactions via $(\gamma, p)$ and $(\gamma, n)$. Additionally, the DOE IP recently established a nuclear data effort specific to isotope production over the next 7 to 8 years to gather proton-induced nuclear data between Lawrence Berkeley National Laboratory (LBNL), Los Alamos National Laboratory (LANL), and Brookhaven National Laboratory (BNL) over the range of 0 to $200 \mathrm{MeV}$. There are ongoing efforts to look at high-energy neutron cross sections in ENDF (Evaluated Nuclear Data File) for certain $(n, p)$ reactions, photonuclear reactions, and low-energy proton- and deuteron-induced reactions of interest.

The High Flux Isotope Reactor (HFIR) at Oak Ridge National Laboratory (ORNL) is one of the workhorses of the system where the product catalog is produced. The DOE IP has also established a network of university isotope producers that complement the traditional national laboratory production sites. This coordinated effort diversifies the portfolio and, in some cases, accelerates access of important isotopes to the user community. Product requests should be submitted to the National Isotope Development Center (NIDC) through www.isotopes.gov. Researchers should also be supplying their current and five year projected isotope needs up to the cognizant program managers at the agency providing them with federal funding.

DOE IP has significant nuclear data needs going forward. Ongoing considerations include target fabrication knowledge capture to maximize time spent on nuclear data collection and analysis. While funding is currently committed for specific projects, other projects will not be excluded as these investments have a direct impact on products and services that the program can provide. Additionally, the program is open to collaborations to accelerate or add scope to current activities. 


\section{A.4.1.2 International Atomic Energy Agency (IAEA)}

The IAEA is working to provide a complete nuclear data library for medical isotope production. This library will soon be available and drives the Medical Isotope Browser, currently available at www.ndsiaea.org/mib. This resource captures production of certain isotopes of interest given defined beam parameters and irradiation conditions as well as contaminants. Building this database depends on two essential parts: high-quality cross section data, and complete and reasonable quality cross section data for all reactions, isomers, ground states, exit channels, and incident particles.

Where data are available, a precise analysis of cross section data has been performed for reaction cross section channels surrounding diagnostic/therapeutic nuclides and monitor reactions via IAEA Coordinated Research Projects (CRPs), generating evaluated data for $\sim 150$ high-quality nuclear reaction channels [1-5]. Approximately 10 to 15 recent unpublished IAEA reaction evaluations still need to be included. A short description of further effort needed is provided below.

Experimental data are typically only complete up to $\sim 40$ to $45 \mathrm{MeV}$. Where data are not available, TENDL (TALYS-based Evaluated Nuclear Data Library) is normalized to the point where there is evaluated experimental data. TALYS-generated data contains uncertainties from model calculations and spreads far beyond experimental data ranges.

The TALYS model has areas for improvement in its predictive capabilities, and currently TALYS only provides predictions up to incident alpha particles. Furthermore, there is a very limited ability to model isotope production from high flux, high-energy secondary particles, e.g. neutrons, due to limitations in modeling high-energy pre-equilibrium reactions. High-energy neutron excitation functions must be included since charged particle-induced cross sections alone will not provide sufficient information for comprehensive production predictions. To extend TALYS to non-standard projectiles (e.g. Li-7), the standard optical model must be replaced with a fusion model to capture heavy ion reactions. It is unclear how difficult this would be to implement, as even incident deuterons are difficult to model.

The intent over the next two years is to perform parameter and model adjustments using Bayesian optimization and machine learning techniques. For these adjustments, protons, photons, and deuterons are priority.

Arjan Koning, Head of the IAEA Nuclear Data Section and the lead TALYS developer, made three clear requests of the experimental community to help improve the code's predictive abilities. The first was that publications report data for all exit channels from high-energy proton irradiations rather than just a few channels of the greatest interest for a specific application. The second was a request for direct measurements of the secondary spectrum. Third, sensitivity and uncertainty analysis information is needed. Specifically, what uncertainty information and analyses are needed to produce a quality product, and to what fidelity.

Session attendees recommended that the IAEA provide a framework for international cooperation on a coordinated data project, similar to what was recently done for the photonuclear library. While TALYS and TENDL are excellent resources, a specifically focused effort may be necessary to address needs beyond what TALYS and TENDL currently provide, and only a coordinated theoretical and experimental effort can address what is needed for isotope production. 


\section{A.4.2 Nuclear Data Capabilities and Needs}

\section{A.4.2.1 Lawrence Berkeley National Laboratory (LBNL)}

\section{Capabilities to support nuclear data}

The nuclear data group at LBNL uses proton, deuteron, and heavy ion beams to address the nuclear data needs of the applied and basic science community. This includes performing stacked target activation measurements using monitor foils interspersed with aluminum degraders together with variance minimization procedures to measure energy-differential charged particle cross sections.

The data group has also explored the use of thick target deuteron breakup on beryllium targets to create an extremely high flux, forward-focused neutron beam, allowing for conversion of $\sim 10 \%$ of incident deuterons into fast neutrons. This approach allows for simultaneous production utilizing both the primary deuteron and secondary neutron beams. The neutron fluence is quantified using activation as well as a suite of scintillators capable of performing in-beam neutron spectroscopy via time-of-flight.

\section{Current Efforts}

As part of the tri-lab effort between LBNL, LANL, and BNL mentioned above, proton-induced reactions on arsenic and niobium have been measured up to $200 \mathrm{MeV}$. Production of the Ge-68 and Se-72 generators and establishing the ${ }^{93} \mathrm{Nb}(\mathrm{p}, 4 \mathrm{n}){ }^{90} \mathrm{Mo}$ reaction for use as a high-energy charged particle monitor were the focus of this work, but a total of 60 ground states and 7 isomers were measured via activation. The next priority is irradiation of antimony foils to characterize production of Te-119m/g and Sn- $117 \mathrm{~m}$.

For all projects the LBNL group makes extensive use of students, providing invaluable training to young scientists. This includes a long-lasting collaboration with the University of Oslo as well as with UC Berkeley students.

\section{A.4.2.2 Facility for Rare Isotope Beams (FRIB) at Michigan State University (MSU)}

\section{Nuclear Data Capabilities}

At the Facility for Rare Isotope Beams (FRIB), heavy ion beams (primary beams of $\mathrm{Ca}-48, \mathrm{Ca}-40, \mathrm{U}-$ 238), are shot through a specifically designed thin target, sending outgoing reaction products through a mass spectrometer to isolate a specific secondary beam for experimental applications [6].

Targets need to be transmissive for forward momentum, meaning that $90 \%$ of the primary beam doesn't react with the target material, going to a flowing water beam stop and depositing $\sim 400 \mathrm{~kW}$ of beam power. With up to $200 \mathrm{MeV}$ per nucleon, there are many interesting reactions occurring in the water. Specific isotopes can be chemically extracted for various applications - e.g. recently, via a flowing water-target [7] at the National Superconducting Cyclotron Laboratory (NSCL), bombardment-induced $\mathrm{Ca}-47$ was extracted and purified to serve as a generator for Sc-47 for radiolabeling experiments.

\section{Nuclear Data Needs}

With regards to nuclear data, there is a need to predict the nuclide yields produced by FRIB beams on water for incident heavy ion particle energies ranging from 0-200 $\mathrm{MeV}$ per nucleon. Nuclear data considerations include: the huge energy range over which these reactions can occur, very high reaction probabilities, and the geometrical cross section leading to $10-30 \%$ loss of beam to nuclear reactions. This means that secondary reactions are much more probable than normally assumed, particularly in the form of very fast forward-focused neutrons. The neutron flux could be probed via activation experiments. 
It is challenging to predict and solve what is being created as the beam is consumed through the target, but some amount of predictive capability is necessary for both safety and health reasons. The fragmentation code (LISE++) over and under predicted by a factor of ten in many cases. Fusion evaporation predictions from PACE4 and LISEfus were also off by an order of magnitude [8]. This demonstrates a significant lack of accurate models and nuclear data. Getting the isomer to ground state ratios for the same exit channel could be very important to assess optical model potentials (OMP) and level density and would be very useful for comparing with predictive capabilities. It is worth noting that optimizing isotope production at FRIB and at existing high-energy proton accelerators involves improvements in nuclear reaction modeling in the same multi-hundred $\mathrm{MeV} /$ nucleon energy range where pre-equilibrium reaction modes play a leading role.

With these improvements, it would be possible to pull out substantial amounts of pure radioactive target material from the beam stop for use by the nuclear data community.

\section{A.4.2.3 Oak Ridge National Laboratory (ORNL)}

\section{Nuclear data capabilities at the High Flux Isotope Reactor (HFIR)}

The High Flux Isotope Reactor has one of the highest fluxes in the world at over $10^{15} \mathrm{n} / \mathrm{cm}^{2} / \mathrm{s}$ within the flux trap. HFIR is an optimum facility to perform in-reactor nuclear data measurements because it has a stable flux within a cycle and from cycle to cycle. There are also two pneumatic tubes with greater than $10^{14} \mathrm{n} / \mathrm{cm}^{2} / \mathrm{s}$ flux that are easily accessible and relatively inexpensive to use. ORNL has created a highfidelity validated transport and depletion model using adjoint methods to reduce uncertainties in target depletion analysis. Currently, LDRD funds are being used to validate the flux spectrum in the experimental irradiation locations, develop a sensitivity analysis of depletion calculations, and conduct integral nuclear data cross section experiments [9].

\section{Nuclear data needs for reactor production of isotopes}

\section{Model Development:}

At HFIR, nuclear data needs are driven by a desire to optimize isotope production to conserve valuable target material and to reduce overall production costs. This optimization is dependent upon highfidelity transport and depletion models using accurate nuclear data to predict the performance of target materials, flux filters, irradiation location, irradiation time, and target heating.

Improvements are needed to the HFIRCON code specific to obtaining higher fidelity cross section measurements. Understanding and improving the target backing, experimental design, and material information are all important for development of nuclear data target production, irradiation, and postirradiation analysis.

\section{Cross Section Needs:}

Cross sections of interest for heavy isotope production include Bk, Cf, Th, and Fm [10]. A code based on empirical data are currently being used, but the embedded cross sections are only applicable for a specific target, location, and set of irradiation conditions. As a result, the cross sections are not globally correct. In addition to cross sections, gamma production data are very important as gamma-induced fission and subsequent target heating must be accounted for. Many of the minor actinide cross sections in ENDF are based on theory and a few experimental data points. Some are missing entirely. For ORNL's reactor models, TENDL theoretical cross sections are used, but experimental data must be obtained to validate the theoretical values. 


\section{Infrastructure Needs:}

Infrastructure is required to support nuclear data experiments. Beam experiments can be performed to obtain differential cross sections on longer lived isotopes at the Lead Slowing-Down Spectrometer (LSDS) at Rensselaer Polytechnic Institute (RPI). In-reactor experiments are the only viable method for isotopes with shorter half-lives and provide the integral of two-group cross sections (thermal and resonance integral). Effective, scenario-specific cross sections can be measured, but flux-independent cross sections are more globally applicable. To obtain this data an accurate characterization of the flux and high-fidelity models are needed.

In addition, improvements are being made to the post-irradiation counting infrastructure. At HFIR, four HPGe Compton suppressed clovers would reduce the background from highly activated targets as well as uncertainties. This system would be extremely helpful to measure fission yields and activation products. A detector located within the hot cell could be used to get down to a timescale of seconds postirradiation for activation measurements.

Once these experimental nuclear data capabilities have been established there will be infrastructure available for the community to measure short-lived actinides and activation products.

\section{A.4.2.4 Argonne National Laboratory (ANL)}

\section{Nuclear data needs in the Radioisotope Research and Production Program (R2P2)}

ANL has a Low Energy Accelerator Facility (LEAF), which is a $20-50 \mathrm{MeV}$ electron linac $(\sim 10-25$ $\mathrm{kW}$ beam power) with three beamlines for photonuclear production, one of which is used for ${ }^{100} \mathrm{Mo}(\gamma, \mathrm{n}){ }^{99} \mathrm{Mo}$ for NNSA and one which is dedicated to radioisotope production for the DOE IP.

The current gold standard (photonuclear reactions on gold) is excellent near the giant dipole resonance at $\sim 15 \mathrm{MeV}$ but is poor beyond this range $(>20 \mathrm{MeV})$. Further, the little experimental data that is available beyond $20 \mathrm{MeV}$ varies greatly. Recently the IAEA published a photonuclear data library. This library contains a great deal of excellent data on the $(\gamma, n)$ reaction channels but none on the $(\gamma, p)$ channels, which ultimately lead to the ability to isolate high specific activity radioisotopes from the target material and are of more interest to the field of nuclear medicine. In general, there are not enough discrete energy-dependent cross section measurements and the results diverge significantly from the theoretical data. Bremsstrahlung makes getting precise energy-dependent cross sections complicated and stacked foil techniques are not possible as photons do not degrade as they pass through a target. However, this does allow for activation of multiple materials in a single irradiation. Concentric target rings were used for collection of multiple energy points in a single irradiation at the Duke High Intensity Gamma-Ray Source (HIGS) facility. This facility produces monoenergetic photons by Compton backscatter when the beam is collimated. If the collimator is removed, the beam provides concentric rings of discrete photon energies. The cross sections obtained thus far for $(\gamma, \mathrm{p})$ channels at the HIGS facility are higher than that predicted by TENDL.

Also located at ANL is the Argonne Tandem Linac Accelerator System (ATLAS), which is a superconducting ion linac with $\sim 10-20 \mathrm{MeV}$ per nucleon for the lighter heavy ion beams. At this facility, "heavy" light ion reactions are being examined for auger-emitter production. There is very little available data in the EXFOR database for reactions induced by helium beams and essentially none for reactions induced by lithium beams.

In terms of nuclear data, both photonuclear and light ion data are needed as the available nuclear data are very limited, non-existent, or inaccurate. Calibration data to be used as monitor reactions are also needed. 


\section{A.4.2.5 Los Alamos National Laboratory (LANL)}

\section{Capabilities to support nuclear data measurements and evaluation}

Los Alamos National Laboratory hosts both the Los Alamos Neutron Science Center (LANSCE) accelerator complex as well as extensive radiochemical processing and characterization facilities for wide-ranging applications to nuclear data, including isotope production and research. The LANSCE accelerator produces proton beams from $100-800 \mathrm{MeV}$, including a $>200 \mu \mathrm{A}, 100 \mathrm{MeV}$ beam directly employed for isotope production. The $800 \mathrm{MeV}$ proton beam is primarily used for neutron production, with white, time-of-flight neutron beams from sub-thermal to over $200 \mathrm{MeV}$, covering over nine orders of magnitude in neutron energy. These beams are delivered to a range of target stations allowing differential cross section measurements of capture, fission, elastic and inelastic scattering, fission product yields, and $(\mathrm{n}, \mathrm{p})$ and $(\mathrm{n}, \alpha)$ reactions. Simultaneous measurement capabilities allow well over 10,000 hours/year of experiments from internal and external proposers.

In addition to neutron beam measurements, the direct proton beam can be accessed for specific nuclear data experiments both at the $100 \mathrm{MeV}$ target station [11,12] as well as at energies $40-200 \mathrm{MeV}$ and $800 \mathrm{MeV}$ [13-15]. Finally, there are extensive counting capabilities for activation-production measurements, allowing access to radioisotopes with lifetimes well below one day.

Nuclear data needs for accelerator-based isotope production

There is still a clear need to improve nuclear data related to isotope production, especially at energies above $70 \mathrm{MeV}$. In order to improve the predictive codes that production sites rely on for planning and safety evaluations, it is necessary to measure not only cross sections of isotopes of interest but also all the associated production channels open at the energy chosen for bombardment.

\section{A.4.3 Nuclear Data Target Production Capabilities and Needs}

The second topic for the session was the current status of materials and targetry for nuclear data experiments. A need was identified for a single target repository, and efforts to ensure that any relevant users have been made aware that this repository exists. Users could make lists of desired targetry materials to inform the nuclear data and isotope production community of what is needed. It should be noted that the DOE IP regularly sends out surveys to determine the community needs and users are encouraged to communicate any need through this mechanism.

The requirements for target fabrication and characterization span a broad range, but are largely driven by the needs of the particular measurement of interest. Important items to consider include the chemistry of the element under study, the physical form and geometry required for the measurement, and in some cases mitigation of hazards associated with the target material itself. As an example, many of the nuclear data measurements currently being conducted by the U.S. national laboratories require actinide targets that vary in mass from micrograms to several hundred milligrams. In almost all cases they require high levels of elemental and isotopic purity to reduce interferences in the data, and a high degree of physical uniformity to control sources of uncertainty associated with the experimental geometry. As experimental equipment and techniques continue to advance, the details of the target are becoming a leading source of uncertainty in many new measurements.

Unfortunately, many of the capabilities that used to exist in the U.S. nuclear physics and nuclear chemistry community to produce and characterize targets have slowly disappeared over the past 30+ years. This has occurred due to many factors, including loss of personnel, facility closures, and a general failure to maintain community knowledge. To address this the community needs to identify what expertise resides where, and work to train the next generation of scientists so that this expertise is not lost in the future. It is also important for these capabilities to be maintained across multiple sites as an incentive for healthy competition and collaboration to keep the field vital. A target knowledge database 
going back to the 1980s exists within the proceedings of the International Nuclear Target Development Society (INTDS). However, there should be a comprehensive compilation of existing and emerging capabilities for both stable and radioactive targetry.

\section{A.4.3.1 Lawrence Berkeley National Laboratory (LBNL)}

\section{Nuclear data targetry capabilities and needs}

There is a balancing act between making targets that are suitable for nuclear data measurements and providing acceptable statistics, i.e., suitably thin targets to minimize energy loss. Availability of quality targets is another issue, e.g., it was not possible to obtain arsenic targets of sufficient quality for a set of nuclear data measurements, so a fabrication technique had to be developed in-house.

The goal of the in-house technique was to fabricate an arsenic target that was approximately 25-50 $\mu \mathrm{m}$ in thickness and common fabrication techniques were not accessible. The targets could not be cast as As sublimes upon heating, it is highly toxic, and cold/hot rolling and extrusion cause cracking. Vapor deposition was also not possible as the target either had pinholes or cracked from stress. Electroplating capabilities were developed at LBNL with arsenic masses ranging from $2-17 \mathrm{mg} / \mathrm{cm}^{2}(1-10 \mu \mathrm{m})$ successfully plated onto $10 \mu \mathrm{m}$ Ti foil.

This struggle to both find and optimize targetry highlights challenges faced by many within the nuclear data community when obtaining high-quality nuclear data.

\section{A.4.3.2 Oak Ridge National Laboratory (ORNL)}

\section{Nuclear data targetry capabilities at the Radiochemical Engineering Development Center (REDC)}

The Isotope Research Materials Lab (IRML) facility at ORNL was shut down in 1990. IRML produced radioisotope targets using methods such as vacuum evaporation, electrodeposition, ion beam sputtering and metal reduction. There have been recent efforts to try to recapture these resources at the Radiochemical Engineering Development Center (REDC).

High-quality thin film targetry remains important to support nuclear data and other experiments, and work is being done to improve uniformity and reduce uncertainties. Improvements in electrodeposition methods are currently being funded via the DOE IP as is ink jet printing development. Ink jet printing of targetry has significant advantages in terms of automated control, high efficiency, uniform deposition, and good material recovery, but it is challenging to design and operate within a glove box or hot cell and ink formulation presents some challenges. Characterization of targetry is important as quantifying target mass, isotopics, chemical form, and uniformity results in reduced experimental uncertainties.

\section{A.4.3.3 Argonne National Laboratory (ANL)}

\section{Nuclear data targetry capabilities at the Center for Accelerator Target Science (CATS)}

This facility has four main objectives: to serve the low-energy community by either producing targets or pointing to people who can, train investigators and students in targetry fabrication to fulfill future community needs, carry out research and development (R\&D) activities dedicated to the development of novel production techniques and optimization of existing ones, and develop an inventory of targets that can serve as a repository available to the general community.

This facility has a wide array of capabilities including electron beam vacuum deposition, thermal resistive heating vapor deposition, sputtering, mechanical rolling, film casting, powder development, and 
molecular plating of actinide targets. A robust counting facility for nuclear data applications is also located in-house.

The current ANL target library rules are that individual target requests will be limited to one specified target and up to two targets shipped. Priority is given to domestic requests and DOE investigators. There is a significant amount of ongoing support for facilities at MSU and CERN.

Communication can be facilitated through the CATS website: www.anl.gov/phy/center-foraccelerator-target-science or by email at cats@anl.gov. The target library is up and running and a publication is forthcoming in an APS conference journal. A target knowledge database does exist within the proceedings of the International Nuclear Target Development Society (INTDS). This information can be probed by searching their website at www.intds.org and contains proceedings and references to targetry work going back to the 1980s.

\section{A.4.3.4 Los Alamos National Laboratory (LANL)}

\section{Nuclear data targetry capabilities}

LANL maintains robust radiochemistry and radiometric counting capabilities that are frequently leveraged for target fabrication and characterization. Commonly used techniques include various methods of electrodeposition (the day-to-day workhorse), stippling, pressed pellets, and more recently, a restored capability to prepare some targets by vapor deposition. Other methods are still the subject of R\&D efforts, such as the use of ink jet printing technologies to lay down very controlled quantities of material in specific geometries. Each of the above also requires appropriate material preparation, dissolution, separations, etc., which are routinely performed in LANL chemistry labs using both stable and radioactive materials.

Thorough characterization of the starting material and the finished target, including accurate uncertainties to include in the final error analysis, are equally important to the entire process. Radiometric measurements are the standard for radioactive materials, but mass spectrometry is necessary to determine isotopic abundances for both stable and radioactive targets. The latter is important as both a stand-alone result as well as a confirmation for radiometric analyses. The Nuclear \& Radiochemistry Group at LANL maintains considerable radiometric counting and mass spectrometry facilities covering a broad range of capabilities. There is also access to considerable physical analysis equipment, both inside and outside the group, including various forms of microscopy (e.g. SEM and AFM) and significant spectroscopic analysis capability.

\section{Radioactive nuclear data targetry capabilities and needs}

There is a strong need for nuclear data supporting nuclear astrophysics. Nucleosynthesis of heavy elements in core-collapse supernovae and in particular in proton-rich trajectories of the neutrino-driven wind scenarios presents an exciting opportunity since the key reaction rate measurements can only be performed with neutrons interacting with a radioactive target. The LANL Isotope Production Facility (IPF) is in a unique position to offer this capability not less because of its proximity to the Weapons Neutron Research (WNR) facility, making experiments with days-short half-life isotopes possible. Once this unique capability has been successfully developed in the United States, it will place U.S. Nuclear Science in a uniquely competitive leadership position worldwide. Radioactive targets have been produced at IPF and used for astrophysics measurements and nuclear reaction studies beyond stability at the Lujan center and the WNR facility.

The development of radioactive targets for quantitative measurements like the ones needed in astrophysics and specific nuclear physics applications is challenging as it requires producing thin, homogenous, and highly uniform targets with highly radioactive material. LANL's unique expertise is considered critical for success in this nuclear data area and it is essential to continue supporting this R\&D 
effort to capitalize on this exciting new capability. Overall, the development of radioactive targets for nuclear astrophysics and nuclear physics represents a unique opportunity to tap on two resources at the same LANSCE accelerator to produce nuclear data that would be unobtainable elsewhere.

Electrodeposition is currently used to plate radioactive materials produced at IPF into targets that are subsequently exposed to a neutron flux at WNR. Microjet plating capabilities are being developed in order to plate uniform radioactive targetry. This set-up is designed such that it can easily survive within a hot cell environment.

Radioactive target characterization presents its own unique set of challenges as these techniques have to be able to operate and reside within hot cells due to the highly radioactive target materials. To deal with thin-layer characterization, the community is exploring new techniques developed by nanomaterial scientists as radioactive targets are often made with extremely small quantities of material.

\section{A.4.4 Conclusion}

There is a real need for nuclear data supporting isotope production, but also targetry development in order to obtain this data.

Identifying cross section priorities, acquiring high-quality cross section data, and improving and adding modeling capabilities have been identified as the top priorities for improving nuclear data in the isotope production community. Experimental data are needed to improve and expand existing predictive models (e.g. charged particle interactions), but also to inform the development of new predictive models for emerging interactions of interest (e.g. photonuclear and heavy ion interactions).

In the spirit of acquiring high-fidelity cross section data, fabrication of high-quality nuclear data targets, identification of current fabrication capabilities, and consolidating available target materials into a single widely known repository, are also priorities for the isotope production community. Radioactive targetry is needed to obtain certain types of nuclear data and presents its own unique set of challenges.

The national laboratories together with universities in the DOE Isotope Program need to continue outreach to the nuclear data community to understand needs and work to fill the gaps in capabilities. 


\section{A.4.5 References}

[1] E. Betak, A.D. Caldeira, R. Capote, B.V. Carlson, H.D. Choi, F.B. Guimaraes, A.V. Ignatyuk, S.K. Kim, B. Kiraly, S.F. Kovalev, E. Menapace, A.L. Nichols, M. Nortier, P. Pompeia, S.M. Qaim, B. Scholten, Y.N. Shubin, J.-C. Sublet, T. F., Nuclear Data for the Production of Therapeutic Radionuclides, Vienna, Austria, 2011.

[2] A. Hermanne, A. V Ignatyuk, R. Capote, B. V Carlson, J.W. Engle, M.A. Kellett, T. Kibedi, G. Kim, F.G. Kondev, M. Hussain, O. Lebeda, A. Luca, Y. Nagai, H. Naik, A.L. Nichols, F.M. Nortier, S. V Suryanarayana, S. Takacs, F.T. Tarkanyi, M. Verpelli, Reference Cross Sections for Chargedparticle Monitor Reactions, Nucl. Data Sheets. $148 \quad$ (2018) 338-382. https://doi.org/10.1016/j.nds.2018.02.009.

[3] J.W. Engle, A. V. Ignatyuk, R. Capote, B. V. Carlson, A. Hermanne, M.A. Kellett, T. Kibédi, G. Kim, F.G. Kondev, M. Hussain, O. Lebeda, A. Luca, Y. Nagai, H. Naik, A.L. Nichols, F.M. Nortier, S. V. Suryanarayana, S. Takács, F.T. Tárkányi, M. Verpelli, Recommended Nuclear Data for the Production of Selected Therapeutic Radionuclides, Nucl. Data Sheets. 155 (2019) 56-74. https://doi.org/10.1016/j.nds.2019.01.003.

[4] F.T. Tárkányi, A. V. Ignatyuk, A. Hermanne, R. Capote, B. V. Carlson, J.W. Engle, M.A. Kellett, T. Kibedi, G.N. Kim, F.G. Kondev, M. Hussain, O. Lebeda, A. Luca, Y. Nagai, H. Naik, A.L. Nichols, F.M. Nortier, S. V. Suryanarayana, S. Takács, M. Verpelli, Recommended nuclear data for medical radioisotope production: diagnostic gamma emitters, J. Radioanal. Nucl. Chem. 319 (2019) 487-531. https://doi.org/10.1007/s10967-018-6142-4.

[5] F.T. Tárkányi, A. V. Ignatyuk, A. Hermanne, R. Capote, B. V. Carlson, J.W. Engle, M.A. Kellett, T. Kibédi, G.N. Kim, F.G. Kondev, M. Hussain, O. Lebeda, A. Luca, Y. Nagai, H. Naik, A.L. Nichols, F.M. Nortier, S. V. Suryanarayana, S. Takács, M. Verpelli, Recommended nuclear data for medical radioisotope production: diagnostic positron emitters, J. Radioanal. Nucl. Chem. 319 (2019) 533666. https://doi.org/10.1007/s10967-018-6380-5.

[6] E.P. Abel, M. Avilov, V. Ayres, E. Birnbaum, G. Bollen, G. Bonito, T. Bredeweg, H. Clause, A. Couture, J. Devore, M. Dietrich, P. Ellison, J. Engle, R. Ferrieri, J. Fitzsimmons, M. Friedman, D. Georgobiani, S. Graves, J. Greene, S. Lapi, C.S. Loveless, T. Mastren, C. Martinez-Gomez, S. McGuinness, W. Mittig, D. Morrissey, G. Peaslee, F. Pellemoine, J.D. Robertson, N. Scielzo, M. Scott, G. Severin, D. Shaughnessy, J. Shusterman, J. Singh, M. Stoyer, L. Sutherlin, A. Visser, J. Wilkinson, Isotope harvesting at FRIB: Additional opportunities for scientific discovery, J. Phys. G Nucl. Part. Phys. 46 (2019). https://doi.org/10.1088/1361-6471/ab26cc.

[7] K.A. Domnanich, E.P. Abel, H.K. Clause, C. Kalman, W. Walker, G.W. Severin, An isotope harvesting beam blocker for the National Superconducting Cyclotron Laboratory, Nucl. Instruments Methods Phys. Res. Sect. A Accel. Spectrometers, Detect. Assoc. Equip. 959 (2020) 163526. https://doi.org/10.1016/j.nima.2020.163526.

[8] E.P. Abel, H.K. Clause, G.W. Severin, Radiolysis and radionuclide production in a flowing-water target during fast 40Ca20+ irradiation., Appl. Radiat. Isot. 158 (2020) 109049. https://doi.org/10.1016/j.apradiso.2020.109049.

[9] J. Knowles, S. Skutnik, D. Glasgow, R. Kapsimalis, A generalized method for characterization of U235 and $\mathrm{Pu}-239$ content using short-lived fission product gamma spectroscopy, Nucl. Instruments Methods Phys. Res. Sect. A Accel. Spectrometers, Detect. Assoc. Equip. 833 (2016) 38-44. https://doi.org/10.1016/j.nima.2016.06.112.

[10] J.B. Roberto, C.W. Alexander, R.A. Boll, J.D. Burns, J.G. Ezold, L.K. Felker, S.L. Hogle, K.P. Rykaczewski, Actinide targets for the synthesis of super-heavy elements, Nucl. Phys. A. 944 (2015) 
99-116. https://doi.org/10.1016/j.nuclphysa.2015.06.009.

[11] A.S. Voyles, L.A. Bernstein, E.R. Birnbaum, J.W. Engle, S.A. Graves, T. Kawano, A.M. Lewis, F.M. Nortier, Excitation functions for $(p, x)$ reactions of niobium in the energy range of $E p=40$ $90 \mathrm{MeV}$, Nucl. Instruments Methods Phys. Res. Sect. B Beam Interact. with Mater. Atoms. 429 (2018) 53-74. https://doi.org/10.1016/j.nimb.2018.05.028.

[12] K. V. Becker, E. Vermeulen, C.J. Kutyreff, E.M. O’Brien, J.T. Morrell, E.R. Birnbaum, L.A. Bernstein, F.M. Nortier, J.W. Engle, Cross section measurements for proton induced reactions on natural La, Nucl. Instruments Methods Phys. Res. Sect. B Beam Interact. with Mater. Atoms. 468 (2020) 81-88. https://doi.org/10.1016/j.nimb.2020.02.024.

[13] J.W. Weidner, S.G. Mashnik, K.D. John, F. Hemez, B. Ballard, H. Bach, E.R. Birnbaum, L.J. Bitteker, A. Couture, D. Dry, M.E. Fassbender, M.S. Gulley, K.R. Jackman, J.L. Ullmann, L.E. Wolfsberg, F.M. Nortier, Proton-induced cross sections relevant to production of 225Ac and 223Ra in natural thorium targets below $200 \mathrm{MeV}$, Appl. Radiat. Isot. 70 (2012) 2602-2607. https://doi.org/10.1016/j.apradiso.2012.07.006.

[14] J.W. Engle, S.G. Mashnik, J.W. Weidner, L.E. Wolfsberg, M.E. Fassbender, K. Jackman, A. Couture, L.J. Bitteker, J.L. Ullmann, M.S. Gulley, C. Pillai, K.D. John, E.R. Birnbaum, F.M. Nortier, Cross sections from proton irradiation of thorium at $800 \mathrm{MeV}$, Phys. Rev. C - Nucl. Phys. 88 (2013) 1-8. https://doi.org/10.1103/PhysRevC.88.014604.

[15] J.W. Engle, S.G. Mashnik, L.A. Parker, K.R. Jackman, L.J. Bitteker, J.L. Ullmann, M.S. Gulley, C. Pillai, K.D. John, E.R. Birnbaum, F.M. Nortier, Nuclear excitation functions from 40 to $200 \mathrm{MeV}$ proton irradiation of terbium, Nucl. Instruments Methods Phys. Res. Sect. B Beam Interact. with Mater. Atoms. 366 (2016) 206-216. https://doi.org/10.1016/j.nimb.2015.10.049. 


\section{A.5 SCATTERING, TRANSPORT AND SHIELDING}

\section{Session Leaders:}

- Matt Devlin, LANL

- $\quad$ Mike Zerkle, NNL

- Andrew Ratkiewicz, LLNL

\section{A.5.1 Introduction}

The third Workshop for Applied Nuclear Data Activities (WANDA) was held at the Elliot School of International Affairs in Washington DC from March $3^{\text {rd }}-5^{\text {th }}, 2020$. A working group was convened to discuss the capabilities and nuclear data needs for codes calculating neutron transport, scattering, and shielding (neutronics codes). These codes play an essential role in the nuclear data pipeline; they are often the first customers of nuclear data, and their results are frequently used to assess the quality of the data. The goals of this session were to discuss: (a) current and future needs for neutronics code capabilities, (b) the nuclear data required to support those capabilities, (c) a subset of current experimental capabilities to meet these nuclear data needs. Based on these discussions, several recommendations will be made in this document.

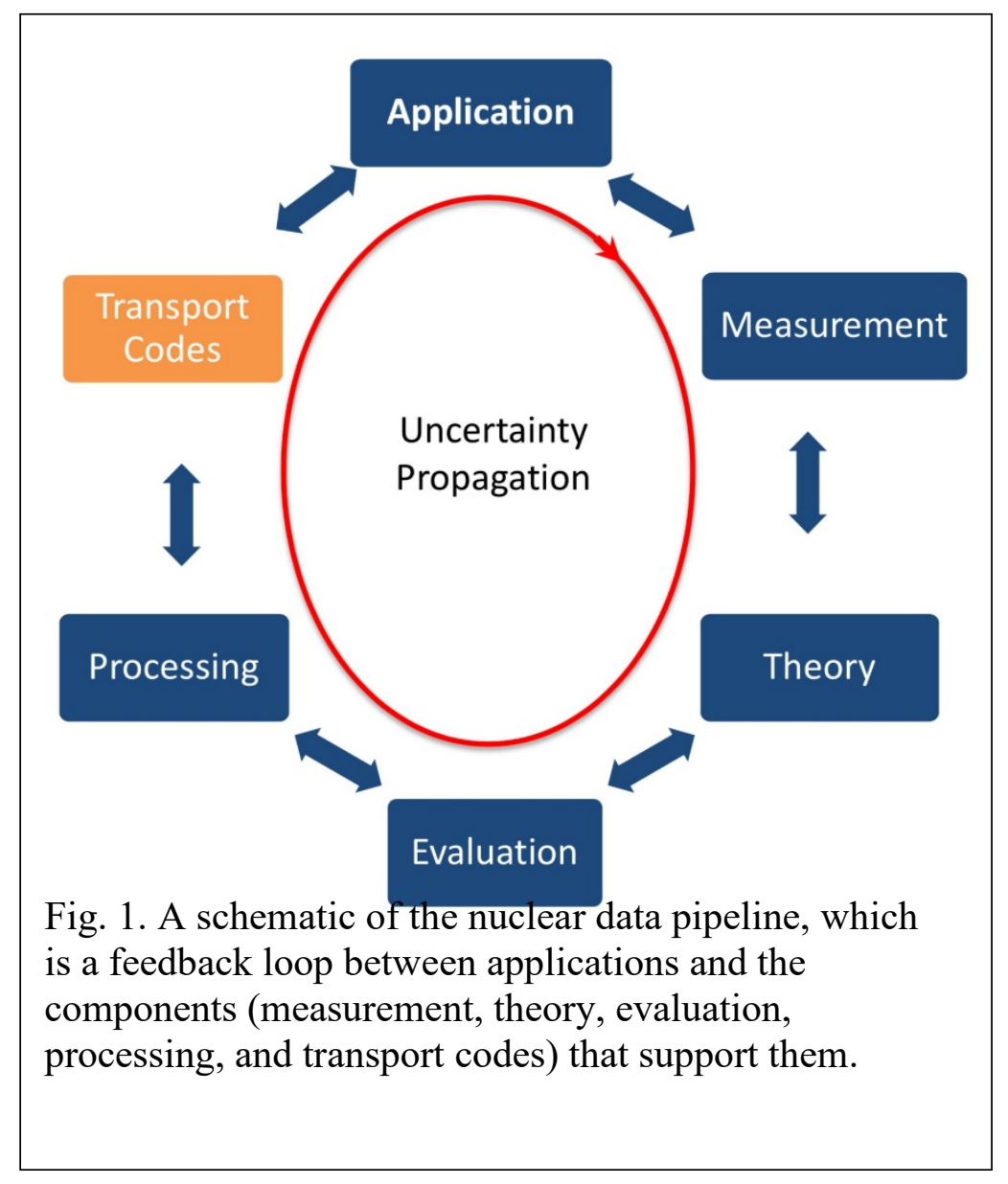


Neutronics codes play key roles in research for basic science and societal applications. As the models these codes simulate become more complex and precision requirements increase, the nuclear data libraries grow, and the calculations become more computationally expensive.

Neutronics codes are increasingly being ported to High Performance Computing (HPC) architectures, which allow much faster calculations with more elaborate models and the consideration of more complicated physics than has been previously accessible. This effort requires validated, high fidelity nuclear data. Acquiring this data are an involved process, which requires experiments sensitive to different physical quantities and processes, their theoretical interpretation, and the expert evaluation of the results of these efforts. The nuclear data must also be processed into a format readable by the neutronics codes, which can often be used to identify deficiencies in nuclear data requiring new or high-precision measurements. Robust Validation and Verification (V\&V) and uncertainty propagation are essential in all steps of this process. The nuclear data pipeline, which includes these steps, is illustrated in Fig. 1. This working group discussed several important components of the nuclear data pipeline, focusing on the neutronics codes and a small subset of recent improvements in nuclear data provided by existing experiments.

\section{A.5.2 Agenda}

Six talks (three on codes, three on experiments) were presented over two sessions to address these topics. The remainder of this document will discuss high-level takeaways from each session and the subsequent discussions and provide recommendations for future work.

\section{A.5.3 Session One Highlights - Neutron Transport Codes:}

\section{Speakers: Jon Dahl (LANL), Will Wieselquist (ORNL), Michael Zerkle (Naval Nuclear Laboratory)}

The talks regarding transport codes gave an overview of a subset of those managed by US National Labs and the Naval Nuclear Laboratory. These talks and subsequent discussion led to the following conclusions regarding the current status and future direction of neutron transport codes and the data which they use:

- Current codes show the need for and the utility of performing Validation and Verification (V\&V) of transport methods and nuclear data at all steps of the nuclear data pipeline. Robust V\&V can identify errors and the stage in the pipeline at which they have been introduced; it is essential for reliable operation of transport codes.

$\circ$ Efforts to include V\&V in every step of the nuclear data pipeline should continue and be encouraged.

- As codes are ported to High Performance Computing (HPC) architectures there is increasing tension between data fidelity and performance. An early step in a neutron transport calculation is reading in the nuclear data libraries, which, due to their large size, can be a bottleneck.

- Computations are faster on HPC architectures than on traditional systems, but disk operations are still expensive. They should be minimized when possible. 
- There may be an opportunity to speed up calculations without sacrificing data fidelity by using Machine Learning (ML) models which have parameterized the necessary nuclear data and can provide physics-on-the- fly.

- Codes should not spend time parsing data - instead this should be done pre-calculation, and the processed data made available by an Application Programming Interface (API) which provides a common set of data to all codes. This will also reduce the chance that codes use slightly different interpretations of nuclear data.

$\circ$ Robust V\&V is an essential component of these upgrades.

The discussion and talks also brought up some important questions, which should be explored:

- It is unclear whether the existing incident neutron energy resolution on fission product yield data are sufficient for advanced reactor applications. Additional energy resolution may be needed in the intermediate and fast energy range to support several small modular reactor concepts.

O The Advanced Reactor community should be asked for their nuclear data needs.

- Neutron-capture data often needs improvement; the full capture gamma cascade is often absent from a library, and an average used instead. This is sufficient for some applications (such as shielding), but not for others (e.g., detector modeling).

○ Gamma energy distributions and multiplicities must also be given, or 'Kerma' (the kinetic energy released in matter) will be incorrect. Accurately predicting Kerma continues to be a challenge, and one that affects neutronics applications.

- Over the last decade there have been significant advances in radiation shielding computational methods. However radiation shielding requirements are still largely based on measurements done in the 1950s and '60s. This results in overconservative shielding design/analysis uncertainties that do not allow applications to take full advantage of the improved accuracy the new radiation shielding methods are providing.

$\bigcirc$ These measurements, benchmarks, and the resulting design/analysis uncertainties should be revisited, and, where necessary, modern experimental techniques and detectors employed to improve the data.

○ Modern, well-characterized shielding benchmarks are needed to support the validation of modern radiation shielding methods for common shielding materials.

\section{A.5.4 Session Two Highlights - Experiments to Improve Nuclear Data:}

Speakers: Ayman Hawari (North Carolina State University), Yaron Danon (Rensselaer Polytechnic Institute), Carl Brune (Ohio University).

The experimental talks gave an overview of nuclear data evaluation methods and experiments probing the properties of neutron-induced reactions important to nuclear data applications.

Ayman Hawari of North Carolina State University gave an overview of work evaluating neutron Thermal Scattering Law (TSL) data, an important aspect of neutron transport in many applications, particularly nuclear reactors. These methods are advancing the state of the art for simulating critical systems. There has recently been a renaissance in this important nuclear 
data area. Modern TSL evaluation methods based on atomistic simulation methods were used to develop over 20 thermal scattering law evaluations for ENDF/B-VIII.0. We now have the evaluation tools in place to generate TSL for practically any moderator of interest. Some measurements are still needed to validate the theoretically generated thermal scattering laws.

Fast neutron elastic and inelastic scattering data are known to be insufficient. Yaron Danon of Rensselaer Polytechnic Institute discussed recent fast neutron scattering experiments, designed to measure the neutron response of nuclei to fast neutrons. In addition to elastic and inelastic scattering cross sections, angular distributions of scattered neutrons are needed for transport calculations. These measurements have been instrumental in identifying issues with evaluated nuclear data libraries and were used to inform nuclear data evaluations for ENDF/B-VIII.0.

Quasi-integral measurement techniques of this type are very powerful and complement the validation provided by critical experiment.

Inelastic neutron scattering in iron, a common material in many systems, have been an issue in the nuclear data evaluation community for some time. Carl Brune of Ohio University discussed some recent fast neutron scattering results using pulsed spheres, in which the integral effect of scattering cross sections and angular distributions can be compared to simulations using various nuclear data libraries. The results imply that neutron elastic and inelastic scattering cross sections in iron are not well described by the usual evaluations, and suggest further work to address the issues.

These talks highlighted the benefits of university-based research, which can provide high- quality measurements and often have access to unique facilities. These talks, and the subsequent discussions, highlighted the opportunity for mutually beneficial partnerships between universities and national laboratories.

- Work done at Universities is important for many reasons, not least that educating young scientists to execute benchmark-quality measurements help to supply the talent pipeline to National Labs.

○ Smaller-scale projects at Universities have contributed, and, given reasonably stable support, will continue to contribute to the national effort in nuclear data for applications. When properly supported they can be more cost effective than national laboratories.

- An important aspect of University work is a close collaboration with National Labs and other users of nuclear data: there is often a need for both guidance about the most urgent nuclear data needs, and for political support within Universities, to show students that there is a career path for this type of work and to convince other faculty that these topics are important.

- A pipeline of trained personnel is required to support the National Labs' mission to execute scientific work in the national interest. This pipeline depends on the Universities, and University-Lab partnerships are essential to maintain it. 


\section{A.5.5 Conclusions and Recommendations}

- The advantages offered by HPC architectures to neutronics codes should be maximized by minimizing disk operations, but without compromising data fidelity.

- This might be achievable through machine-learning models trained to provide physics-on-the-fly, rather than reading in large amounts of nuclear data.

- When possible, nuclear data should be provided to neutronics codes through an API.

$\bigcirc$ This will prevent errors induced by different data preparation strategies in different neutronics codes.

$\bigcirc$ This will help to minimize the amount of time neutronics codes need to spend parsing data.

- Robust Verification and Validation is essential in every stage of the nuclear data pipeline. It should continue to be encouraged and performed.

- There has been a renaissance in the thermal neutron scattering. Thermal neutron scattering law evaluations can now be produced for practically any material of interest.

- New radiation shielding validation benchmarks are needed in order to take full advantage of the improved accuracy of modern radiation shielding methods.

- Non-traditional quasi-integral nuclear data measurement techniques are needed to inform nuclear data for shielding and other applications where neutron scattering effects are important.

- Many communities use nuclear data. However, these communities have historically been compartmentalized, and experimentalists measuring nuclear data are not always aware of their current and evolving needs.

$\bigcirc$ Efforts should be made to increase communication between communities using nuclear data and the communities supplying it. WANDA is an excellent forum for these conversations.

- Stable funding of identified, important nuclear data projects, from measurement through evaluation and dissemination, is needed to maximize the utility of nuclear data efforts.

- Universities are essential members of the community supplying nuclear data. They educate the next generation of scientists, perform measurements that cannot be executed elsewhere, and drive innovative research.

$\circ$ The partnerships between Universities and National Labs are critical to filling the nuclear science talent pipeline the Labs rely on. These partnerships should continue to be supported and strengthened.

- Communication between the National Labs and Universities regarding nuclear data needs, experimental and theoretical capabilities, and data quality requirements is an essential component of these partnerships.

$\circ \quad$ The capabilities and expertise offered by University labs are often unique and irreplaceable. These essential capabilities must be maintained.

○ The pipeline of graduates from University programs in nuclear data and applications is required to support the National mission to in nuclear science and engineering. 


\section{A.6 NEUTRON INDUCED GAMMA PRODUCTION AND GAMMA DECAY}

\section{Session Leaders:}

- Lee Bernstein, LBL, UCB

- Alejandro Sonzogni, BNL

- Amanda Lewis, UCB (student)

\section{A.6.1 Introduction}

The goals of the WANDA 2020 Gamma-ray production session were to:

1. Review the role played by neutron-induced $\gamma$-ray, e.g., $(n, x \gamma)$, production data in a variety of applications (talks by Ayllon, Mauborgne, McConchie and Ressler);

2. Determine areas of the nuclear data pipeline where improvements are needed, e.g., data collection, compilation, evaluation/processing (talks by McCutchan, Nobre and Vogt);

3. Review existing and needed capabilities to address these nuclear data needs (talks by Bleuel and Lewis), and;

4. Create a plan (e.g., a roadmap) as to how to address the most important of those needs.

Several different types of $(\mathrm{n}, \mathrm{x} \gamma)$ data were considered in this analysis, including thermal neutron capture, fast neutron interactions (including capture, inelastic and non-elastic channels) and prompt/isomeric $\gamma$-ray spectra from $(n, f)$. It should be noted that this session did not consider $\alpha$ - or $\beta$-delayed gamma decay data.

\section{A.6.2 Experimental Facilities Overview}

The session opened with an overview of existing $(\mathrm{n}, \mathrm{x} \gamma)$ experimental capabilities by Dr. D.L. Bleuel He pointed out that there were numerous facilities throughout the world with neutron sources and spectrometers with complementary capabilities. His talk included a comparison between the neutron source and spectrometer capabilities at different labs and detailed descriptions of the spectrometer systems at eight "representative" facilities: LANSCE/Chi-nu; the Gaerttner LINAC (RPI); neutron ELBE (DresdenRossendorf); the University of Kentucky Accelerator Lab/KEGS; the Triangle University Nuclear Labs at Duke; the Edwards Lab (Ohio University); the GAINS spectrometer at GELINA (Geel); and the GENESIS array at LBNL. These locations cover monoenergetic (Kentucky, Ohio, TUNL), broad (LANSCE, RPI, GELINA) and narrow/adjustable (LBNL) time-of-

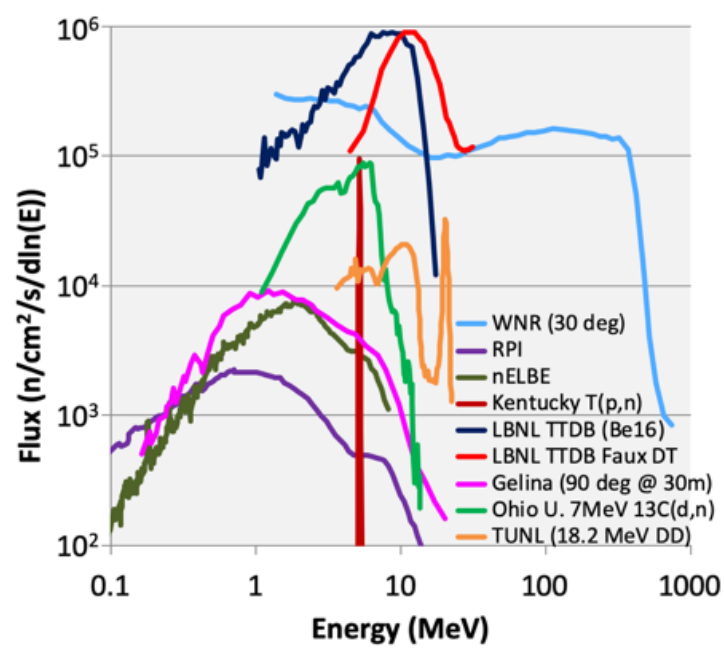

Figure A.1. Neutron flux at the various facilities presented by Bleuel.

flight ranges. Figure A.1 shows the flux at several locations for all these facilities. Dr. Bleuel stated that a recurring issue was a lack of quantitative information about these facilities to allow for a complete comparison.

It was noted that while many facilities had the ability to measure either scattered neutrons or $\gamma$-ray production, relatively few facilities, such as the GENESIS array at LBNL and the newly enhanced Chi-nu facility are developing efforts to measure both simultaneously and in coincidence. 


\section{A.6.3 Applications dependent on $(n, x \gamma)$ data}

There were several recurring themes that occurred throughout the session. The first was that it was difficult-to-impossible to disentangle neutron scattering from neutron-induced $\gamma$-ray production in applications involving fast, e.g., prompt fission neutron spectrum (PFNS) and D(T,n)a (DT) neutrons. This was particularly clear for the four examples presented by Ayllon, Mauborgne, McConchie and Ressler that involved active interrogation. While this topic was discussed in a separate, simultaneous session at the workshop, it is likely that many of the needs discussed in both sessions would overlap. Any evaluated neutron scattering cross sections used to model applications such as neutron active interrogation that fail to accurately reproduce measured $\gamma$-ray production cross sections could be indicative of compensating uncertainties ${ }^{15,16}$ that would impact transport as well.

Active interrogation systems are generally comprised of a neutron source (usually DT), one or more $\gamma$-ray detection systems, and a modeling/analysis capability that interprets the data to determine the composition of an irradiated sample. The neutron sources discussed included compact, high-intensity, broad angle DT systems, used for applications ranging from oil well logging (Mauborgne), to determining the nutrient content of soil and elemental composition of the environment surrounding an interplanetary probe (Ayllon), to low-rate "tagged" neutron sources using the Deuterium-Tritium Associated Particle Imaging (DT-API) technique where the outgoing $\alpha$-particle from the $\mathrm{D}(\mathrm{T}, \mathrm{n}) \alpha$ reaction is detected and used to identify the outgoing neutron " $\mathrm{t}=0$ " and angle (McConchie).

The gamma-detection systems included low- to intermediate-energy-resolution scintillators as well high-resolution systems capable of resolving transitions between discrete low-lying nuclear states. should be noted that even in the case of high-resolution detector systems a good knowledge of not only the partial cross sections populating the discrete states, but also the continuum components of the $\gamma$ spectrum is necessary since it forms a high-energy coincident background that needs to be included in any realistic interpretation of the data.
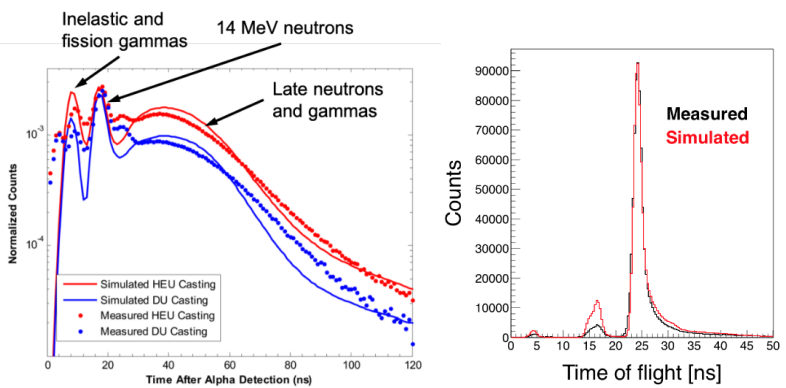

Figure A.2. Examples of nuclear data deficiencies in the modeling of the time-dependent gamma-ray signal from pulsed neutron scattering using a $14 \mathrm{MeV}$ DTAPI source using MCNP-PoliMi (left) and GEANT 4 (right) from the talk by McConchie.

In the talks by Ayllon and McConchie numerous examples were given of specific deficiencies involving $\left(\mathrm{n}, \mathrm{n}^{\prime} \gamma\right)$ to low-lying discrete states in both fissionable/fissile nuclides and even ubiquitous lighter materials such as $\mathrm{C}, \mathrm{O}$ and Al. Figure A.2 shows deficiencies in modelling the time-dependent neutron and $\gamma$-ray signals from irradiation of depleted uranium and highly-enriched uranium castings using both the MCNP-PoliMi and GEANT simulation packages. In the case of the lighter nuclides these deficiencies could be attributed in large part to the lack of energy-differential $\gamma$-ray partial cross section data. Two of these examples from the talk by Ayllon are shown in Fig. A.3.

\footnotetext{
${ }^{15}$ E. Bauge et al., Eur. Phys. J. A (2012) 48: 113. https://doi.org/10.1140/epja/i2012-12113-7

${ }^{16}$ L.A. Bernstein et al., Annu. Rev. Nucl. Part. Sci. 2019. 69:109-36. https://doi.org/10.1146/annurev-nucl-101918$\underline{023708}$
} 

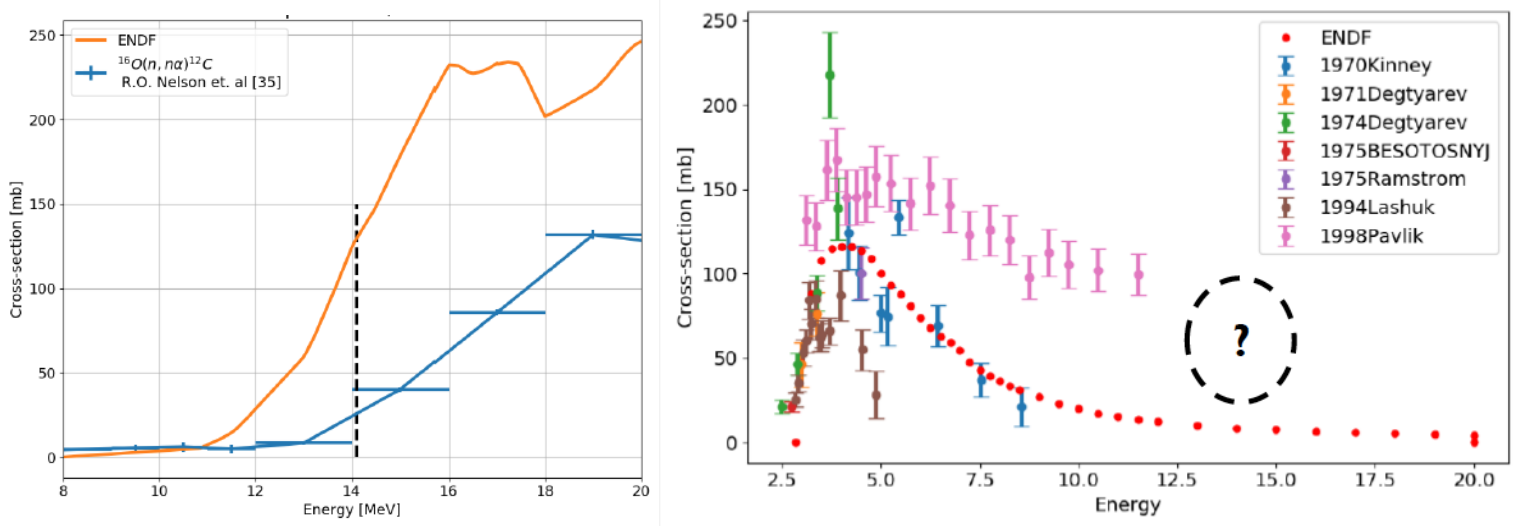

Figure A.3. Data for the production of the $4.4 \mathrm{MeV} \gamma$-ray in ${ }^{12} \mathrm{C}$ from the ${ }^{16} \mathrm{O}(\mathrm{n}, \mathrm{n}$ ' $\alpha \gamma)$ reaction (left) and the $1.7 \mathrm{MeV} \gamma$-ray from ${ }^{27} \mathrm{Al}\left(\mathrm{n}, \mathrm{n}^{\prime} \gamma\right)$ (right) from the talk by Ayllon. (What is the big ? supposed to represent?)

In addition to the reactions shown in Figure A.3, Ayllon and Mauborgne found significant differences between ENDF versions; the latest ENDF /B-VIII.0 performing the worst in comparison with experiments, probably due to the change related to breaking natural compounds into individual isotopes after ENDF /B-VI. Mauborgne's talk focused primarily on issues in neutron capture $\gamma$-ray production, highlighting that more recent versions of ENDF were generally less successful than older versions. These are summarized in Table A.1 in the Appendix. A recurring issue that she brought up, that was echoed by other session attendees, was that the move from elemental to isotopic $\gamma$-ray production data in ENDF (which occurred after ENDF-B6) led to an overall decrease in data quality. In addition to pointing out issues in $(\mathrm{n}, \gamma)$ data her talk she pointed to issues on modeling ${ }^{\text {nat }} \mathrm{Mg}$ and ${ }^{\text {nat }} \mathrm{Si}(\mathrm{n}, \mathrm{n}$ ' $\gamma)$.

In addition to the issues relevant to active interrogation presented by Ayllon, Mauborgne and McConchie Dr. J.J. Ressler from LLNL discussed a number of other applications that were highly dependent on neutron capture $\gamma$-ray production ranging from emergency response to high resolution $\gamma$-ray spectrometers on NASA missions to Mercury, Psyche, Phobos and Titan, to Electromagnetic Pulse and asteroid deflection systems. She also brought up the importance of ${ }^{155,157} \mathrm{Gd}(\mathrm{n}, \gamma)$ data for the interpretation of data from the WATCHMAN detector system which utilizes Gd-doped water to detect antineutrinos from nuclear reactors. It should be noted that in the case of this application the users

\begin{tabular}{|l|l|l|l|}
\hline Element & ENDF-B/VI & ENDF/B-VII.1 & ENDF/B-VIII.0 \\
\hline Hydrogen & $\checkmark$ & $\checkmark$ & $\checkmark$ \\
\hline Silicon & $\checkmark$ & $\checkmark$ & $\checkmark$ \\
\hline Calcium & $\checkmark$ & $?$ & $?$ \\
\hline Iron & $\checkmark$ & $\checkmark$ & $?$ \\
\hline Manganese & $\checkmark$ & $\checkmark$ & $?$ \\
\hline Magnesium & $\checkmark$ & $?$ & $?$ \\
\hline Titanium & $\checkmark$ & $\checkmark$ & $?$ \\
\hline Sodium & $\checkmark$ & $\checkmark$ & $\checkmark$ \\
\hline Chlorine & $\checkmark$ & $\checkmark$ & $\checkmark$ \\
\hline Aluminum & $\checkmark$ & $\checkmark$ & $\checkmark$ \\
\hline
\end{tabular}

Table A.1: Comparison between measured and modelled $(\mathbf{n}, \gamma)$ spectra for 10 elements. Green checks $(\checkmark)$ indicate good agreements while red question marks (?) questionable reproduction of measurements from the talk by Mauborgne. modified the data themselves, using DICEBOX simulation package to get better $\gamma$-multiplicity, and saw improved results. The need for DICEBOX simulations to create a realistic $\gamma$-multiplicity shows that not only $(n, \gamma)$ between discrete states, but also the effects of transitions in the quasi-continuum is needed. Neither the CapGam nor EGAF databases contains this information 
The problems presented in these talks emphasize the recurring issue of poor communication between the application and nuclear data communities. "Customers" from applications dependent on thermal (n, $\gamma)$ data have expressed these issues at the Cross Section Evaluation Working Group (CSEWG) meeting, which is the principle venue for communicating nuclear data aresues. Furthermore, modeling (n, $\gamma)$ is technically challenging for a number of reasons. Firstly, there is a lack of high-quality capture data at all energies above thermal. This is unfortunate since $\gamma$-ray spectra are highly dependent on angular momentum, which is in turn is very sensitive to the energy of the incoming neutron. Furthermore, the continuum-to-discrete approach at a defined cut-off energy used to model primary capture gamma energies and intensities is unphysical. In short, there has been insufficient support to provide evaluators with the data and tools they need to properly model the capture gamma spectrum as function of neutron energy.

\section{A.6.4 Database resources and needs}

Three talks were given that directly addressed prompt $(\mathrm{n}, \mathrm{x} \gamma)$ data. Dr. E.A. McCutchan, , pointed to over 100 examples of inconsistences in prompt, thermal $(n, \gamma)$ transitions linking discrete low-lying states between the two "flagship" databases maintained by the US Nuclear Data Program: ENSDF and ENDF. There is a clear need to reconcile these differences and ensure that the correct data are present in both databases. Participants in the session pointed out that there are two database sources available for prompt thermal $(\mathrm{n}, \gamma)$ data - The CapGAM ${ }^{17}$ library maintained at the NNDC and the EGAF ${ }^{18}$ database hosted at the IAEA. The EGAF library was last evaluated in 2003, while CapGAM is continuously updated with new $(n, \gamma)$ data. CapGam is a complete discrete library, containing all $(n, \gamma)$ measurements, whereas EGAF provides a complementary subset of isotopes limited to those measured at the Budapest reactor under consistent experimental conditions. Not only is there a need to reconcile the differences among the libraries, there are also many cases where the $(n, \gamma)$ data are decades old and taken with very primitive detection systems. There is a clear need to re-measure key isotopes with modern gamma-ray spectrometers to verify and improve upon the current data available in these libraries.

The primary and secondary $\gamma$-rays in both CapGAM and EGAF are for thermal neutron capture. Dr. Toshihiko Kawano from LANL pointed out that capture $\gamma$-ray spectra are expected to change dramatically with increasing neutron energy due to changes in the $J^{\pi}$. This could have particularly significant effects on the interpretation of data from active interrogation applications.

Another limitation in these databases is that they only include transitions between discrete levels. This is a significant issue for heavier nuclei where most of the $\gamma$-ray energy emitted from the capture product is likely to be in the form of an energetic continuum. These spectra are generally modeled using continuous level density and radiative strength functions which are a part of the Reference Input Parameter Library maintained by IAEA Nuclear Data Section ${ }^{19}$. Also, the IAEA is now concluding a Coordinated Research Project (CRP) on Photonuclear Data and Photon Strength Functions ${ }^{20}$. This CRP includes some limited data on Average Resonance Capture (ARC) which occurs at higher keV energies and could provide critical input to modeling the change in the $\gamma$-ray spectra as a function of incident neutron energy mentioned by Kawano.

\footnotetext{
${ }^{17}$ https://www.nndc.bnl.gov/capgam/

${ }_{18}$ R.B.Firestone, et al. Nucl. Data Sheets 119, 79 (2014). https://www-nds.iaea.org/pgaa/egaf.html

${ }^{19}$ R. Capote et al., Nuclear Data Sheets - Volume 110, Issue 12, December 2009, Pages 3107-3214. https://wwwnds.iaea.org/RIPL-3/

${ }^{20}$ https://www-nds.iaea.org/CRP-photonuclear/
} 
These problems in capture gamma-ray data do not lend confidence to prompt (n,n' $\gamma$ ) data where there is even less information available. Dr. G. Nobre referred to this lack of data in his talk, pointing out that the vast majority of nuclides lack both the differential data needed to guide the modeling at the core of the evaluation process and the integral benchmarks needed to ensure consistency between different incident and outgoing neutron energies. Dr. Nobre also provided an overview of the (n,n' $\gamma$ ) modeling used in the evaluation process. He pointed out the importance of having correct $J^{\pi}$ values for discrete low-lying states, assumed deformation for Distorted Wave Born Approximation calculations, and proper treatment of the transition from quasi-continuum to discrete levels $\gamma$-ray cascade. The sensitivity of $\left(\mathrm{n}, \mathrm{n}^{\prime} \gamma\right)$ on the $J^{\pi}$ assignment of low-lying levels highlights its use as a tool for nuclear structure studies. Figure A.4 shows
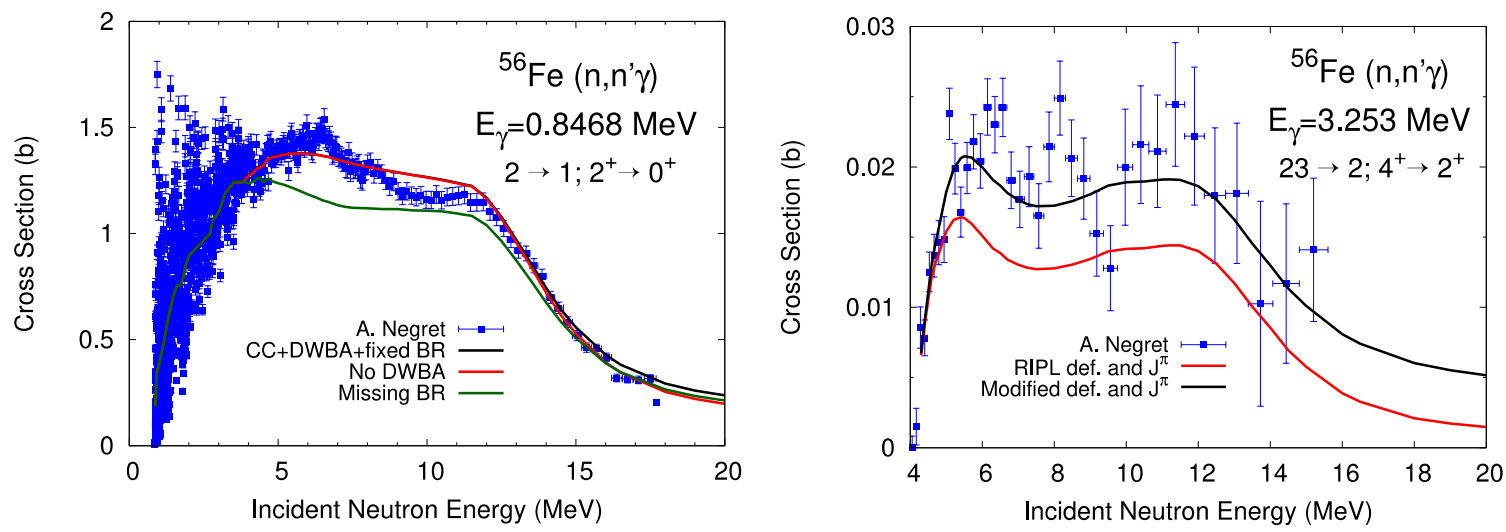

Figure A.4. Comparison between measured and modeled ${ }^{56} \mathrm{Fe}\left(\mathrm{n}, \mathrm{n}^{\prime} \gamma\right)$ transitions for the yrast $2^{+} \rightarrow 0^{+}$ (left) and a more highly excited off-yrast $4^{+} \rightarrow$ yrast $2^{+}$transition (right) highlighting the importance of accurate deformation and spin-parity assignments.

examples from ${ }^{56} \mathrm{Fe}\left(\mathrm{n}, \mathrm{n}^{\prime} \gamma\right)$, which was reevaluated by the BNL/NNDC team as a part of the CIELO collaboration and for which there is a recent high-quality measurement from GELINA by Negret ${ }^{21}$ to which it can be compared. Dr. Nobre also pointed out that the rate at which new evaluations of neutroninduced $\gamma$-ray production cross sections takes place is currently resource-limited.

In her talk Ms. Amanda Lewis described a potential integral benchmark that is being developed for (n,n' $\gamma$ ) data - the Atlas of GammaRays from the Inelastic Scattering of Reactor Fast Neutrons, aka the "Baghdad Atlas"22. The data in the Atlas came from a set of consistent measurements performed in the 1970s at the IRT5000 Research Reactor in Iraq. The Atlas data include the intensities of $7090 \gamma$-rays detected in a single HPGe detector from (n,n' $\gamma$ ) on 75 different elemental and isotopically-enriched targets that were irradiated in a filtered, fast neutron beam line at the reactor. Ms. Lewis described how the neutron spectrum seen by the samples was determined via a $\chi^{2}$-minimization between the

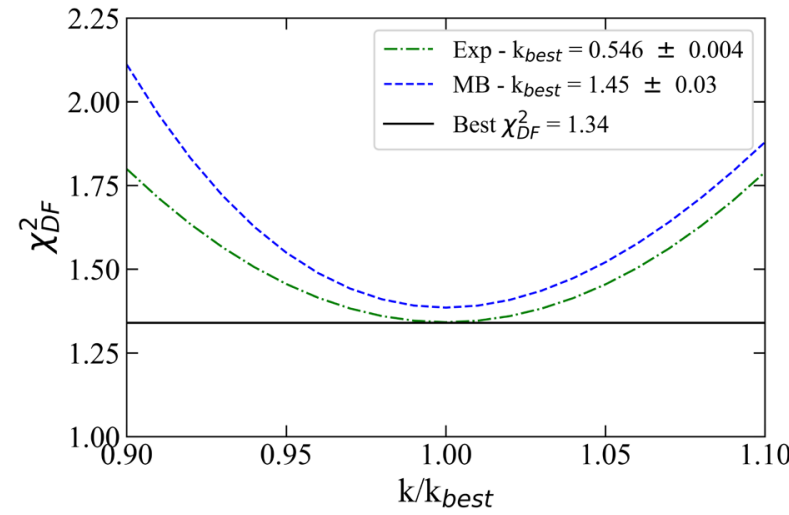

Figure A.5. Goodness of fit between the ${ }^{56} \mathrm{Fe}(\mathrm{n}, \mathrm{n}$ ' $\gamma)$ data in the Atlas and data from and energy differential cross section measurement from GELINA convolved with and exponential and a Maxwell-Boltzmann neutron spectrum.

\footnotetext{
${ }^{21}$ A. Negret et al., Phys. Rev. C 90, 034602 (2014). https://doi.org/10.1103/PhysRevC.90.034602

22 http://nucleardata.berkeley.edu
} 
data in the Atlas for ${ }^{56} \mathrm{Fe}\left(\mathrm{n}, \mathrm{n}^{\prime} \gamma\right)$ and a recent measurement from GELINA ${ }^{7}$ convolved with a neutron spectrum having a Maxwell-Boltzmann and a simple exponential functional form. Both results produced excellent fits with a $\chi^{2}$ per degree of freedom of 1.45 and $1.34 \mathrm{MeV}$ respectively. This fit is shown in Fig. A.5. She also presented a preliminary comparison between the Atlas $\mathrm{Fe}\left(\mathrm{n}, \mathrm{n}^{\prime} \gamma\right)$ partial $\gamma$-ray cross sections in ENDF/B-VIII.0.

The large prompt $\gamma$-ray energy release from (n,f) poses special issues compared to capture and inelastic scattering. In her talk Vogt pointed out that there is little known about neutrons and gammas produced from fission at $14 \mathrm{MeV}$. There are new evaluations of prompt gammas in ENDF/B-VIII.0 for ${ }^{235} \mathrm{U}(\mathrm{n}, \mathrm{f})$, ${ }^{238} \mathrm{U}(\mathrm{n}, \mathrm{f})$ and ${ }^{239} \mathrm{Pu}(\mathrm{n}, \mathrm{f})$ that are a great improvement relative to previous ENDF/B releases, with fixes for some issues such as only finite prompt fission photon multiplicity for incident neutron energies of less than $\sim 1 \mathrm{MeV}$ for ${ }^{235} \mathrm{U}(\mathrm{n}, \mathrm{f})$ in ENDF/B-VII.1. However, the evaluations are still based on rather sparse energy dependent data ${ }^{23}$ and as such it is difficult to validate the energy dependent behavior. The only measurement of the total photon energy at $14 \mathrm{MeV}$ is by Frehaut (1983) and the data on photon multiplicity at this energy are discrepant. This is shown in Fig. A.6. Even for thermal incident neutrons, where the most data are available, the neutron multiplicity measurements disagree by $30 \%$ while the data on total photon energy disagree by up to $50 \%$. However, while the gamma multiplicity distribution has been obtained for thermal neutron-induced fission, the multiplicity distribution at $14 \mathrm{MeV}$ is not known.

In Stetcu et al., where the new evaluations for ENDF/B-VIII.0 were documented, the gamma multiplicity
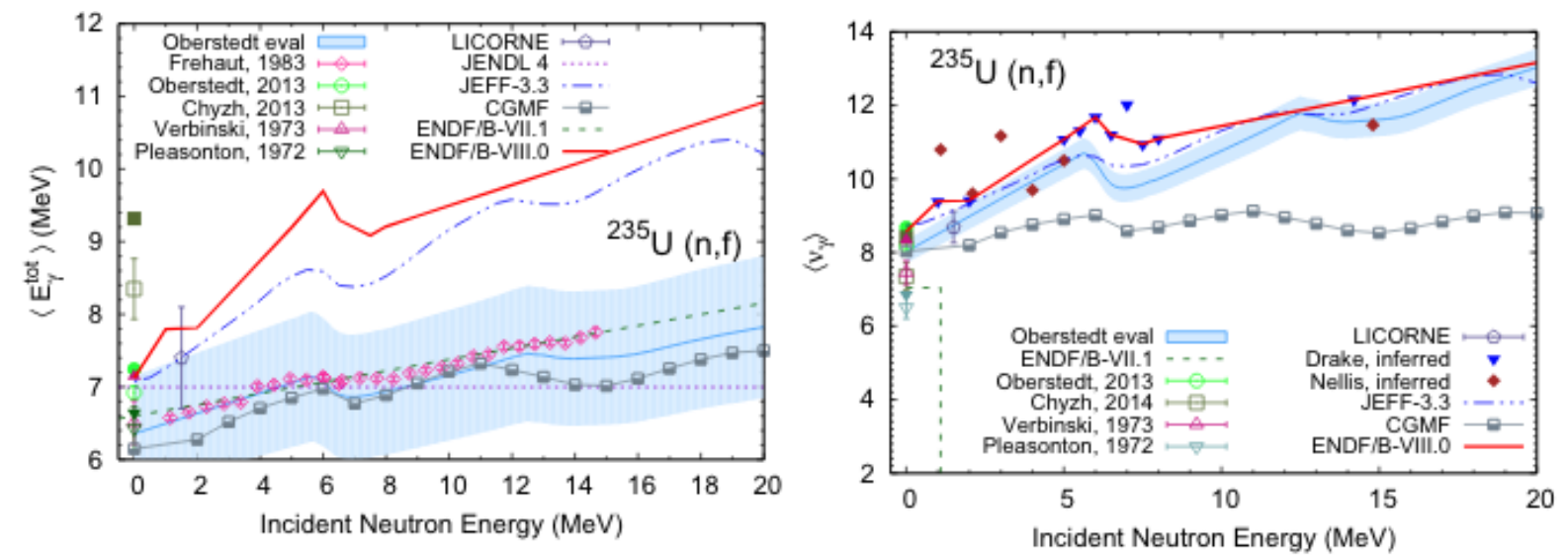

Figure A.6. A comparison measured and modeled values for total photon energy and multiplicity from ${ }^{235} \mathbf{U}(\mathbf{n}, \mathbf{f})$.

distributions were described by a negative binomial distribution with parameters fit to CGMF simulations. The CGMF results, however, are not used for the evaluated averages for total energy, gamma multiplicity, or energy per gamma as a function incident neutron energy. In addition, the energy per gamma is constructed to be constant as a function of neutron energy, inconsistent with the CGMF simulations (which increase with neutron energy) and the Oberstedt evaluation (which decreases). Both results also show threshold behavior for multichance fission, not present in ENDF/B-VIII.0. Average quantities measured by the Oslo group via ${ }^{239} \mathrm{Pu}(\mathrm{d}, \mathrm{pf})$ and ${ }^{233} \mathrm{U}(\mathrm{d}, \mathrm{pf})$ only show a mild excitation energy dependence, albeit at excitation energies below the threshold for second chance fission ${ }^{24}$.

\footnotetext{
${ }^{23}$ I. Stetcu et al., Evaluation of the Prompt Fission Gamma Properties for Neutron Induced Fission of ${ }^{235,238} \mathrm{U}$ and ${ }^{239} \mathrm{Pu}$, Nuclear Data Sheets 163261 (2020).

${ }^{24}$ S. J. Rose et al., Phys. Rev. C 96014601 (2017). https://doi.org/10.1103/PhysRevC.96.014601
} 
Vogt pointed out that some applications may require knowledge of the correlation of prompt $\gamma$-rays with particular fragments, especially for high-yield fragments with strong transitions. There have been measurements of total gamma energy, gamma multiplicity and energy per gamma as a function of fragment mass for thermal neutron-induced fission of ${ }^{233} \mathrm{U}^{25},{ }^{235} \mathrm{U}^{26}$ and ${ }^{239} \mathrm{Pu}^{9,27}$. Generally, these data, taken in the early 1970s, were low statistics and rather inconclusive, in part due to small solid angle coverage for gammas, apart from a single coincident gamma-gamma measurement made using the GEANIE detector for ${ }^{235} \mathrm{U}(\mathrm{n}, \mathrm{f})$ from $1-250 \mathrm{MeV}^{28}$. It would be valuable to repeat these measurements with modern detector arrays that include a fragment detector. Such measurements can lead to improved modeling in complete fission event simulations such as CGMF and FREYA.

Other measurements of discrete gamma transitions from specific fragments are important for fission product yield measurements, particularly as these experiments become more sophisticated. This includes measuring fission product yields at various time scales where different gamma transitions are important. It is possible to make the simulation codes sensitive to these various time windows even though they are not, in themselves, time dependent.

Lastly, there is interest in improved modeling of neutron-gamma correlations which are sensitive to fission properties such as neutron-gamma competition, important for high energy gamma emission, and relative neutron and gamma multiplicities. Measurements of these correlations, both event-by-event and fragment-by-fragment, are sensitive probes of model details.

\section{A.6.5 The Path Forward: A Coordinated Measurement and Evaluation Program}

The consensus amongst the session participants was that a coordinated measurement and evaluation program is needed to provide reliable data needed for active neutron interrogation. The decision was made to form a Gamma Rays Induced by Neutrons (GRIN) task force comprised of experimentalists, modelers, evaluators and programmatic experts that would collaborate to perform the following tasks:

1. Perform targeted measurements of fast and thermal $(n, \gamma)$ and all non-elastic $\gamma$-producing reactions, including most notably $\left(n, n^{\prime} \gamma\right)$, for the most important nuclides that play a role in applications involving active interrogation for terrestrial, space and national security/nonproliferation. These would include correlated energy- and angle-differential cross section measurements in the case of (n,n' $\gamma$ ) and would span an incoming energy region from 14 $\mathrm{MeV}$ down to thermal and include $\gamma$-ray detectors capable of covering the energy range from 50 $\mathrm{keV}$ to $10 \mathrm{MeV}$.

2. Perform integral benchmark experiments that cover the same energy range. These are essential in order to minimize compensating errors between elastic and inelastic scattering ${ }^{1,2}$. Examples of this sort of integral benchmark experiments include the recent work by Daskalakis ${ }^{29}$.

3. Address the inconsistencies in thermal $(n, \gamma)$ data between ENSDF and ENDF pointed out in the talk by McCutchan. This will likely involve both a literature review (compilation) that includes data at all incident neutron energies in addition to thermal, and a physics based modeling effort.

\footnotetext{
${ }^{25}$ F. Pleasonton, Nucl. Phys. A 213, 413 (1973). https://doi.org/10.1016/0375-9474(73)90161-9

${ }^{26}$ F. Pleasonton, R.L.Ferguson, \& H.W. Schmitt, Phys. Rev. C6, 1023 (1972).

https://doi.org/10.1103/PhysRevC.6.1023

27 J.L. Ullmann et al., Phys. Rev. C 87, 044607 (2013). https://doi.org/10.1103/PhysRevC.87.044607

${ }^{28}$ W. Younes et al., Phys. Rev. C 64, 054613. https://doi.org/10.1103/PhysRevC.64.054613

${ }^{29}$ A.M. Daskalakis et al., Ann. Nucl. En. 73 (2014) 455-464. https://doi.org/10.1016/j.anucene.2014.07.023
} 
The goal would be to address these issue for nuclides of greatest importance to applications within 3 years.

4. Establish the Baghdad Atlas as a new energy-integral (integrated?) nuclear data benchmark for gamma-ray production cross sections from $\left(\mathrm{n}_{\text {fast }}, \mathrm{n}^{\prime} \gamma\right)$. This would include reconciling differences in gamma-ray energies between those listed in the Atlas and the most recent version of adopted levels and gammas from ENSDF and an additional benchmarking of the $\chi^{2}$-minimization procedure used determine the neutron spectrum.

5. Use a modeling procedure to produce evaluated $(n, \gamma)$ and $\left(n, n^{\prime} \gamma\right)$ data that includes neutron and $\gamma$ ray correlations. This is the philosophy behind modeling packages such as CGMF ${ }^{30}$. Without this feature the accurate interpretation of active interrogation systems, especially those featuring both scattered neutron and gamma correlations, will have limited value. It should also be noted that the transport codes need to be able to perform correlated emission simulations in order to properly use correlation data. The Generalized Nuclear Data Structure (GNDS) better integrates structure and reaction data and, when coupled to a Monte Carlo nuclear data sampling tool such as MCGIDI, offers an opportunity to ensure that transport simulations sample correlations "in-line". This is already a feature in Mercury and could potentially be used with GEANT as well.

\section{A.6.6 Conclusion}

Neutron-induced $\gamma$-ray production is clearly important for a large number of applications involving neutron-driven (?) active interrogation. However, there are significant issues in almost every component of the nuclear data pipeline with regard to this important class of nuclear data. Furthermore, improved measurements of $\gamma$-ray production over the entire energy range from thermal to $20 \mathrm{MeV}$ supports not only the applications described in this session by Ayllon, Mauborgne, McConchie and Ressler, but also to improved neutron transport relevant to an even wider range of applications including shielding, fast reactor design and broader national security applications.

The community has become aware of this since the inception of the Nuclear Data Working Group (NDWG) and the Nuclear Data Interagency Working Group (NDIAWG), and in late 2018 a joint DOE Nuclear Energy/Office of Science Effort that includes experimental efforts at LBNL and LANL coupled to a modeling effort at BNL/NNDC has been launched to address ${ }^{238} \mathrm{U}\left(\mathrm{n}, \mathrm{n}\right.$ ') for fast reactor design ${ }^{31}$. This included adding high-resolution $\gamma$-ray detectors to the Chi-nu spectrometer at LANL and building the Gamma Energy Neutron Energy Spectrometer for Inelastic Scattering (GENESIS), which is the first-ever array of detectors for the outgoing neutrons and $\gamma$-rays produced in a neutron-induced reaction in coincidence.

These joint measurement-evaluation efforts offer a template for future efforts to improve nuclear data for not only advanced reactor design, but also the terrestrial, interplanetary and national security applications presented in this session.

\section{A.6.7 Application specific needs}

During the writing of this session report a number of the session contributors provided a set of specific isotopic needs for specific applications. While there were many overlapping needs, we have kept the lists separate for organizational purposes.

\footnotetext{
30 P. Talou et al., Eur. Phys. J. A (2018) 54: 9 https://doi.org/10.1140/epja/i2018-12455-0

${ }^{31}$ N.W. Touran et al., Engineering 3 (2017) 518-526. https://doi.org/10.1016/J.ENG.2017.04.016
} 


\section{A.6.7.1 Nuclear Data Needs for Oil Well Logging (M. Mauborgne)}

These are the comments from Dr. M.-L. Mauborgne. They are broken up into specific elements. The check mark $(\checkmark)$ represents a need that has already been met. All comments in bold face are from the original list from Dr. Mauborgne.

- Calcium

- For thermal capture gamma production cross section, significant degradation in ENDF/B-VII and above. This is a major concern for us. (Fig 8 in Mauborgne et al., ${ }^{32}$ )

- For inelastic gamma production cross section, good results with small improvement noticeable in ENDF/B-VII and above.

- Magnesium

- For thermal capture gamma production cross section, ENDF/B-VII and above are useless. This is a major concern for us. (Fig 11 and 12 of Mauborgne et al. ${ }^{33}$,)

- For inelastic gamma production cross section, Line at $1.808 \mathrm{MeV}$ is missing, except that ENDF/B-VII and above are better (Fig 14 Ref, 19). This is a minor concern.

- Iron

- On thermal capture gamma production cross section lots of peak between 2 and $5 \mathrm{MeV}$ have disappeared in the ENDF/B-VIII version (Fig 7 of Ref. 19.) and the peak at $9.297 \mathrm{MeV}$ is missing (from ${ }^{54} \mathrm{Fe}$ ). That is a significant concern for us.

- For inelastic gamma production cross section, it is more difficult as there is very little structure in the response. ENDF/B-VIII seems better below $2 \mathrm{MeV}$ and probably too low after $5 \mathrm{MeV}$. That is a significant concern for us.

- Silicon

- Good thermal capture gamma production cross section. I start to see some discrepancy above $7 \mathrm{MeV} \checkmark$

- For inelastic gamma production the main lines or OK, but the spectrum is too low after the $1.77 \mathrm{MeV}$ peak (Fig 4 of Ref. 19). It seems to be more an issue with the continuum value as shown in Fig 5. That is a significant concern for us.

- Sodium

- For thermal capture gamma production cross section good results with small improvement noticeable in ENDF/B-VII and above $\checkmark$

- For inelastic gamma production cross section, no noticeable change on our application, but peak resolution in the library is very broad. That is a significant concern for us.

- Titanium

- For thermal capture gamma production cross section, significant degradation in ENDF/B-VII and worsen in ENDF/B-VIII (improved peak resolution though) (Fig 15 and 16 of Ref.

19) That is a significant concern for us.

- For inelastic gamma production cross section, good results with small improvement noticeable in ENDF/B-VIII $\checkmark$

- Manganese

- On thermal capture gamma production cross section, significant improvements have been made by Dr Toshihiko Kawano (LANL) following CSWEG meeting under the coordination of Andrej Trkov. After that update, this is not a concern anymore.

- For inelastic gamma production cross section, different releases have similar results. This is a minor concern.

- Hydrogen.
○ Good thermal capture gamma production cross section $\checkmark$

\footnotetext{
${ }^{32}$ M.L. Mauborgne et al., EPJ Web of Conferences 146, 09036 (2017) DOI: 10.1051/epjconf/201714609036

${ }^{33}$ M.L. Mauborgne et al., Proceedings of the Nuclear Data 2019 conference, in press.
} 
- Oxygen

- Good inelastic gamma production cross section $\checkmark$

- Carbon:

- Good inelastic gamma production cross section $\checkmark$

- Aluminum

- For thermal capture gamma production cross section, good results with small improvement noticeable in ENDF/B-VII $\checkmark$

- For inelastic gamma production cross section, no noticeable change on our application, overall good results

- Chlorine

- For thermal capture gamma production cross section, no significant change on our application, overall good results

- For inelastic gamma production cross section, no noticeable change on our application, overall good results

\section{A.6.7.2 Nuclear Data Needs for NASA Dragonfly/BECA mission (M. Ayllon-Unzueta).}

A number of discrepancies in $(\mathrm{n}, \mathrm{x} \gamma)$ data have been found by the group working on the Dragonfly/BECA projects at NASA and cooperating institutes. These reactions are shown in Table 1 below. Note that this list is non-exhaustive and even though it is targeted to the specific geochemistry of the Moon, Saturn's moon Titan, Mars' moon Phobos, and asteroid 16 Psyche, these cross-sections are extensively used for other active interrogation applications on earth such as non-proliferation, drugs and contraband detection, carbon content in soil, etc. This table presents (when available) specific reactions and gamma lines when using the best suited ENDF library.

\begin{tabular}{|c|c|c|c|c|c|c|}
\hline $\begin{array}{l}\text { Target } \\
\text { 1sotope }\end{array}$ & Reaction & $\begin{array}{c}\text { Energy level } \\
(\mathrm{MeV})\end{array}$ & $\begin{array}{r}\text { Gamma } \\
\text { energy } \\
(\mathrm{MeV})\end{array}$ & $\begin{array}{c}\text { Moon }(\mathrm{M}), \text { Titan }(\mathrm{n}, \\
\text { Phobos }(\mathrm{Ph}), \text { Psyche (Ps) }\end{array}$ & $\begin{array}{c}\text { Data Library } \\
\text { (ENDF) }\end{array}$ & Notes \\
\hline $0-16$ & $\left(n, n^{\prime} \alpha\right)$ & $4.439(\mathrm{C}-12)$ & 4.439 & $\mathrm{M}, \mathrm{T}, \mathrm{Ph}$ & $\mathrm{B} 6.8$ & MCNP6 overpredicts*** \\
\hline $0-16$ & $(n, \alpha \gamma)$ & $3.089(\mathrm{C}-13)$ & 3.089 & $\mathrm{M}, \mathrm{T}, \mathrm{Ph}$ & $\bar{B} 6.8$ & MCNP6 underpredicts*** \\
\hline $\mathrm{N}-14$ & $\left(n, n^{\prime} \gamma\right)$ & 2.313 & 2.313 & $\mathrm{~T}$ & $\mathrm{~B} 7.0$ & MCNP6 overpredicts• \\
\hline $\mathrm{C}-12$ & $\left(\mathrm{n}, \mathrm{n}^{\prime} \gamma\right)$ & 4.439 & 4.439 & $\mathrm{~T}$ & B6.8 & $\begin{array}{l}\text { Angular dependent cross- } \\
\text { section } \\
\text { needs reevaluation* }\end{array}$ \\
\hline AI-27 & $\left(\mathrm{n}, \mathrm{n}^{\prime} \gamma\right)$ & 2.734 & 1.72 & $\mathrm{M}, \mathrm{T}, \mathrm{Ps}$ & $\mathrm{B} 6.8$ & MCNP6 underpredicts*** \\
\hline Al-27 & $\left(n, n^{\prime} \gamma\right)$ & 2.212 & 2.212 & $\mathrm{M}, \mathrm{T}, \mathrm{Ps}$ & $\mathrm{B} 6.8$ & MCNP6 underpredicts*** \\
\hline $\mathrm{Si}-28$ & $\left(\mathrm{n}, \mathrm{n}^{\prime} \gamma\right)$ & $6.276->1.779$ & 4.497 & $\mathbf{M}, \mathrm{T}, \mathrm{Ph}, \mathrm{Ps}$ & B6.8 & $\begin{array}{l}\text { Important for correcting } \\
\text { carbon line } \text { e }^{* *}\end{array}$ \\
\hline $\mathrm{Si}-28$ & $(\mathrm{n}, \mathrm{x})$ & & $>6$ & M, T, Ph, Ps & $\mathrm{B} 6$ & MCNP6 underpredict $\mathrm{s}^{* *}$ \\
\hline Fe-56 & $\left(\mathrm{n}, \mathrm{n}^{\prime} \gamma\right)$ & 0.847 & 0.847 & M, T, Ph, Ps & B6.8 & MCNP6 overpredicts*** \\
\hline$\overline{\mathrm{Fe}}-56$ & $\left(n, n^{\prime} \gamma\right)$ & $2.085->0.847$ & 1.238 & $\mathbf{M}, \mathrm{T}, \mathrm{Ph}, \mathrm{Ps}$ & $\bar{B} 6.8$ & MCNP6 underpredicts**** \\
\hline $\mathrm{Cl}-35$ & $(\mathrm{n}, \mathrm{d})$ & $2.127(\mathrm{~S}-34)$ & 2.127 & $\mathrm{~T}$ & B7.0 & MCNP6 overpredicts* \\
\hline Cl-35 & $\left(n, n^{\prime} \gamma\right)$ & 3.163 & 3.163 & $\mathrm{~T}$ & $\mathrm{~B} 7.0$ & MCNP6 underpredicts* \\
\hline $\mathrm{Na}-23$ & $\left(n, n^{\prime} \gamma\right)$ & $2.076->0.440$ & 1.636 & $\mathrm{M}, \mathrm{T}, \mathrm{Ph}$ & $\mathrm{B} 7.0$ & Lar11:e relative error* \\
\hline $\mathrm{Na}-23$ & $(\mathrm{n}, \mathrm{d})$ & $1.275 ! \mathrm{Ne}-21)$ & 1.275 & $\mathrm{M}, \mathrm{T}, \mathrm{Ph}$ & $\overline{B 7.0}$ & MCNP6 underpredicts* \\
\hline $\mathrm{Na}-23$ & $\left(n, n^{\prime} \gamma\right)$ & 0.440 & 0.440 & $\mathrm{M}, \mathrm{T}, \mathrm{Ph}$ & $\mathrm{B} 7.0$ & MCNP6 underpredicts* \\
\hline $\mathrm{Ni}-58$ & $\left(\mathrm{n}, \mathrm{n}^{\prime} \gamma\right)$ & 1.454 & 1.454 & $\mathrm{~T}, \mathrm{Ps}$ & 87,0 & MCNP6 overpredicts* \\
\hline $\mathrm{Ca}-40$ & $\left(n, n^{\prime} \gamma\right)$ & 3.736 & 3.736 & $\mathbf{M}, \mathrm{T}, \mathrm{Ph}, \mathrm{Ps}$ & B7.0 & MCNP6 overpredicts* \\
\hline $\mathrm{Ca}-40$ & $\left(n, n^{\prime} \gamma\right)$ & 3.904 & 3.904 & M, T, Ph, Ps & $\bar{B} 7.0$ & MCNP6 overpredicts* \\
\hline $\mathrm{Ca}-40$ & $(\mathrm{n}, \mathrm{p})$ & $\begin{array}{c}0.800->0.030 \\
\text { IK- } 401\end{array}$ & 0.77 & $\mathbf{M}, \mathrm{T}, \mathrm{Ph}, \mathrm{Ps}$ & B7.0 & MCNP6 overpredicts* \\
\hline $\mathrm{Mg}-24$ & $\left(\mathrm{n}, \mathrm{n}^{\prime} \gamma\right)$ & 4.238 & 4.238 & $\mathrm{M}, \mathrm{T}, \mathrm{Ph}$ & B6 & $\begin{array}{c}\text { MCNP6 underpredicts, } \\
\text { future ENDF releases } \\
\text { wrong** }\end{array}$ \\
\hline
\end{tabular}




\begin{tabular}{|c|c|c|c|c|c|c|}
\hline $\mathrm{Mg}-24$ & $\left(n, n^{\prime} \gamma\right)$ & 1.369 & 1.369 & $\mathrm{M}, \mathrm{T}, \mathrm{Ph}$ & B6 & $\begin{array}{c}\text { MCNP6 underpredicts, } \\
\text { future ENDF releases } \\
\text { wrong** }\end{array}$ \\
\hline $\mathrm{Mg}-24$ & $(\mathrm{n}, \mathrm{d})$ & $\begin{array}{c}2.076->440 \\
(\mathrm{Na}-23)\end{array}$ & 1.636 & $\mathrm{M}, \mathrm{T}, \mathrm{Ph}$ & B6 & $\begin{array}{c}\text { MCNP6 underpredicts, } \\
\text { future ENDF releases } \\
\text { wrong ** }\end{array}$ \\
\hline $\mathrm{Mn}-55$ & $\left(\mathrm{n}, \mathrm{n}^{\prime} \gamma\right)$ & $1.292->0.126$ & 1.166 & $\mathbf{M}$ & B6 & $\begin{array}{c}\text { Future ENDF releases } \\
\text { wrong } * *\end{array}$ \\
\hline S-32 & $\left(\mathrm{n}, \mathrm{n}^{\prime} \gamma\right)$ & 2.23 & 2.23 & T, Ps & $?$ & \\
\hline La-139 & $?$ & $?$ & $?$ & M, T, Ph,Ps & $?$ & Detector material \\
\hline Br-nat & $?$ & $?$ & $?$ & $\mathrm{M}, \mathrm{T}, \mathrm{Ph}, \mathrm{Ps}$ & $?$ & Detector material \\
\hline Ce-nat & $?$ & $?$ & $?$ & $\overline{\mathrm{M}, \mathrm{T}, \mathrm{Ph}, \mathrm{Ps}}$ & $?$ & Detector material \\
\hline Ge-nat & $?$ & $?$ & $\bar{?}$ & $\mathrm{M}, \mathrm{T}, \mathrm{Ph}, \mathrm{Ps}$ & $?$ & Detector material \\
\hline
\end{tabular}

*El. Kanawati 2011, ** Mauborgne 2020, *** Ayllon 2020 
APPENDIX B. MEETING AGENDA AND PRESENTATIONS 


\begin{tabular}{|c|c|c|}
\hline \multicolumn{3}{|c|}{ WANDA2020 AGENDA } \\
\hline & TUESDAY, MAR 3 & \\
\hline 730 & \multicolumn{2}{|l|}{ Registration \& Continental Breakfast } \\
\hline 800 & Welcome \& Opening Remarks & $\begin{array}{l}\text { Tim Hallman, Associate Director of Science for Nuclear } \\
\text { Physics }\end{array}$ \\
\hline 815 & Workshop Structure and Goals & Catherine Romano, ORNL \\
\hline 835 & Nuclear Data for NA-22 Proliferation Detection & $\begin{array}{l}\text { Donny Hornback / Chris Ramos, Office of Proliferation } \\
\text { Detection, DNN R\&D, NNSA }\end{array}$ \\
\hline 855 & Nuclear Data for NA-22 Forensics & $\begin{array}{l}\text { Tim Ashenfelter, Office of Proliferation Detection, } \\
\text { DNN R\&D, NNSA }\end{array}$ \\
\hline 905 & DTRA Nuclear Data Needs & Joanne Ingraham, Defense Threat Reduction Agency \\
\hline 915 & NRC Nuclear Data Needs & Don Algama, Nuclear Regulatory Agency \\
\hline 925 & Office of Nuclear Energy Nuclear Data Needs & David Henderson, Office of Nuclear Energy \\
\hline 935 & Nuclear Criticality Safety Program & Mike Zerkle, Naval Nuclear Laboratory \\
\hline 945 & Naval Reactors Nuclear Data Needs & Steve Bell, Naval Reactors, NNSA \\
\hline 955 & BREAK & \\
\hline 1015 & $\begin{array}{l}\text { The IAEA Nuclear Data programme and Nuclear } \\
\text { Data in } 2040\end{array}$ & $\begin{array}{l}\text { Arjan Koning, Section Head, Nuclear Data Section, } \\
\text { IAEA }\end{array}$ \\
\hline 1030 & Nuclear Energy Agency Nuclear Data Activities & $\begin{array}{l}\text { Arjan Koning for Michael Flemming, Nuclear Energy } \\
\text { Agency, Division of Nuclear Science }\end{array}$ \\
\hline 1045 & Nuclear Data Pipeline - Getting Data to the User & $\begin{array}{l}\text { Panel - Dave Brown, BNL; Yaron Danon, RPI; Patrick } \\
\text { Talou, LANL; Jeremy Conlin, LANL; Teresa Bailey, LLNL; } \\
\text { Michael Zerkle, NNL; Robert Casperson, LLNL }\end{array}$ \\
\hline 1145 & WANDA Successes and Lessons Learned & Lee Bernstein, LBL/UCB \\
\hline \multirow[t]{2}{*}{1200} & \multicolumn{2}{|l|}{ Lunch Group Photo } \\
\hline & \multicolumn{2}{|l|}{ Group Roadmapping Session 1} \\
\hline \multirow{2}{*}{1315} & $\begin{array}{l}\text { Artificial Intelligence and Machine Learning for } \\
\text { Nuclear Data }\end{array}$ & $\begin{array}{l}\text { Vlad Sobes, UTK; Kyle Wendt, LANL; Mike Grosskopf, } \\
\text { LLNL; Dave Brown, BNL; Michael Smith, ORNL; Patrick } \\
\text { Talou, LANL }\end{array}$ \\
\hline & $\begin{array}{l}\text { Detector Models, Atomic Data and Stopping } \\
\text { Powers }\end{array}$ & $\begin{array}{l}\text { Bethany Goldblum, UCB; Bruce Pierson, PNNL; Matt } \\
\text { Devlin, LANL }\end{array}$ \\
\hline 1515 & \multicolumn{2}{|l|}{ Break } \\
\hline 1535 & \multicolumn{2}{|l|}{ Group Roadmapping Session 1 (continued) } \\
\hline \multirow[t]{2}{*}{1715} & Adjourn Day 1 & \\
\hline & WEDNESDAY, MAR 4 & \\
\hline \multirow[t]{2}{*}{730} & \multicolumn{2}{|l|}{ Registration \& Continental Breakfast } \\
\hline & \multicolumn{2}{|l|}{ Group Roadmapping Session 2} \\
\hline \multirow{2}{*}{800} & $\begin{array}{l}\text { Covariance/Sensitivity/Uncertainty/Validation and } \\
\text { Its Impact on Applications }\end{array}$ & $\begin{array}{l}\text { Denise Neudecker, Robert Casperson, Rike } \\
\text { Bostelmann }\end{array}$ \\
\hline & $\begin{array}{l}\text { Nuclear Data for Isotope Production and Nuclear } \\
\text { Data Target Fabrication }\end{array}$ & Etienne Vermulen, Greg Severin, Ellen O'Brien \\
\hline 1000 & \multicolumn{2}{|l|}{ Break } \\
\hline 1020 & \multicolumn{2}{|l|}{ Group Roadmapping Session 2 (continued) } \\
\hline \multirow[t]{2}{*}{1200} & \multicolumn{2}{|l|}{ Lunch } \\
\hline & \multicolumn{2}{|l|}{ Group Roadmapping Session 3} \\
\hline \multirow[t]{2}{*}{1315} & $\begin{array}{l}\text { Neutron Induced Gamma Production and Gamma } \\
\text { Decay }\end{array}$ & $\begin{array}{l}\text { Lee Bernstein, LBL/UCB; Alejandro Sonzogni, BNL; } \\
\text { Amanda Lewis, UCB }\end{array}$ \\
\hline & Scattering, Transport and Shielding & Brian Quiter, LBL; Mike Zerkle, NNL \\
\hline 1515 & \multicolumn{2}{|l|}{ Break } \\
\hline 1535 & \multicolumn{2}{|l|}{ Group Roadmapping Session 3 (continued) } \\
\hline 1715 & \multicolumn{2}{|l|}{ Adjourn Day 2} \\
\hline 1715 & \multicolumn{2}{|l|}{ Optional No Host Social at the Elliott School } \\
\hline
\end{tabular}




\begin{tabular}{|c|c|c|}
\hline & THURSDAY, MAR 5 & \\
\hline 745 & \multicolumn{2}{|l|}{ Registration \& Continental Breakfast } \\
\hline 830 & $\begin{array}{l}\text { The Nuclear Data Interagency Working Group } \\
\text { (NDIAWG) FOA }\end{array}$ & $\begin{array}{l}\text { Tim Hallman, Associate Director of Science for Nuclear } \\
\text { Physics }\end{array}$ \\
\hline 845 & $\begin{array}{l}\text { Improving the Nuclear Data on Fission Product } \\
\text { Decays at CARIBU }\end{array}$ & Guy Savard, ANL \\
\hline 900 & $\begin{array}{l}\text { Novel Approach for Improving Antineutrino } \\
\text { Spectral Predictions for Nonproliferation } \\
\text { Applications }\end{array}$ & Guy Savard for Filip Kondev, ANL \\
\hline 915 & $\begin{array}{l}238 \mathrm{U}(\mathrm{p}, \mathrm{xn}) \text { and } 235 \mathrm{U}(\mathrm{d}, \mathrm{xn}) 235-237 \mathrm{~Np} \text { Nuclear } \\
\text { Reaction Cross Sections Relevant to the Production } \\
\text { of } 236 \mathrm{gNp}\end{array}$ & Lee Bernstein, LBL/UCB \\
\hline 930 & $\begin{array}{l}\text { State-of-the-art Gamma-ray Spectroscopy to } \\
\text { Enhance the ENSDF }\end{array}$ & Elizabeth McCutchan, BNL \\
\hline 945 & $\begin{array}{l}\text { Beta-strength function, reactor decay heat, and } \\
\text { anti-neutrino properties from total absorption } \\
\text { spectroscopy of fission fragments }\end{array}$ & Krzysztof Rykaczewski, ORNL \\
\hline 1000 & $\begin{array}{l}\text { Improving the double-differential } 238 \mathrm{U}\left(\mathrm{n}, \mathrm{n}^{\prime} \mathrm{g}\right) \text { cross } \\
\text { section using neutron-gamma coincidences }\end{array}$ & Lee Bernstein, LBL/UCB \\
\hline \multicolumn{3}{|c|}{1015 BREAK } \\
\hline 1035 & $\begin{array}{l}\text { Integral Measurements of Independent and } \\
\text { Cumulative Fission Product Yields Supporting } \\
\text { Nuclear Forensics and Other Applications }\end{array}$ & Todd Bredeweg, LANL \\
\hline 1050 & $\begin{array}{l}\text { Evaluation of Energy Dependent Fission Product } \\
\text { Yields }\end{array}$ & Toshihiko Kawano, LANL \\
\hline 1105 & $\begin{array}{l}\text { Measurement of Independent Fission Product } \\
\text { Yields }\end{array}$ & Dana Duke, LANL \\
\hline 1120 & $\begin{array}{l}\text { Independent Fission Product Yields from } 0.5 \text { to } 20 \\
\mathrm{MeV}\end{array}$ & Jack Winkelbauer, LANL \\
\hline 1135 & Energy Dependent Fission Product Yields & Anton Tonchev, LLNL \\
\hline 1150 & Nuclear Data Scoping Studies & Catherine Romano, ORNL \\
\hline \multicolumn{3}{|c|}{1200 LUNCH } \\
\hline 1300 & Session Summaries & Session Leaders \\
\hline \multicolumn{3}{|c|}{1500 Break } \\
\hline 1520 & Q\&A Session & Facilitated by Lee Bernstein, LBL/UCB \\
\hline 1650 & Workshop Closing and Next Steps & Catherine Romano, ORNL \\
\hline 1700 & Adjourn Final Day 3 & \\
\hline
\end{tabular}




\section{B.2 WANDA2020 Attendees}

\begin{tabular}{|c|c|c|}
\hline Alexander & Damien & Defense Threat Reduction Agency \\
\hline Algama & Don & United States Nuclear Regulatory Commission \\
\hline Ashenfelter & Timothy & $\begin{array}{l}\text { NNSA/DNN R\&D/Office of Nuclear Detonation } \\
\text { Detection }\end{array}$ \\
\hline Bailey & Teresa & Lawrence Livermore National Laboratory \\
\hline Balkin & Ethan & US Dept. of Energy/NP-DOE Isotope Program \\
\hline Barnes & Mark & Savannah River National Laboratory \\
\hline Baumeister & Jakob & Brookhaven National Laboratory \\
\hline Beck & Bret & LLNL \\
\hline Bell & Stephen & Naval Reactors \\
\hline Benny & Paul & Oak Ridge National Laboratory \\
\hline Bernstein & Lee & LBNL/UC-Berkeley \\
\hline Bevins & James & AFIT \\
\hline Birnbaum & Eva & Los Alamos National Laboratory \\
\hline Blain & Ezekiel & Rensselaer Polytechnic Institute \\
\hline Bledsoe & Keith & Oak Ridge National Laboratory \\
\hline Bleuel & Darren & Lawrence Livermore National Laboratory \\
\hline Bond & Evelyn & Los Alamos National Laboratory \\
\hline Bostelmann & Friederike & Oak Ridge National Laboratory \\
\hline Bredeweg & Todd & Los Alamos National Lab \\
\hline Brown & David & Brookhaven National Laboratory \\
\hline Brune & Carl & Ohio University \\
\hline Bucher & Brian & Idaho National Laboratory \\
\hline Burcher & Sean & Lawrence Livermore National Laboratory \\
\hline Burke & Jason & LLNL \\
\hline Carlson & Allan & $\begin{array}{l}\text { National Institute of Standards and } \\
\text { Technology/Brookhaven National Laboratory }\end{array}$ \\
\hline Casperson & Robert & Lawrence Livermore National Laboratory \\
\hline Chadwick & Mark & Los Alamos National Laboratory \\
\hline Chen & Jun & $\begin{array}{l}\text { National Superconducting Cyclotron } \\
\text { Laboratory, Michigan State University }\end{array}$ \\
\hline Chisolm & Eric & Department of Energy \\
\hline Conlin & Jeremy & Los Alamos National Laboratory \\
\hline Dahl & Jon & Los Alamos National Laboratory \\
\hline Danon & Yaron & Rensselaer Polytechnic Institute \\
\hline deBoer & Richard & University of Notre Dame \\
\hline Descalle & Marie-Anne & Lawrence Livermore National Laboratory \\
\hline Devlin & Matthew & Los Alamos National Laboratory \\
\hline Dinwiddie & Derek & Los Alamos National Laboratory \\
\hline Duke & Dana & Los Alamos National Laboratory \\
\hline
\end{tabular}




\begin{tabular}{|c|c|c|}
\hline Escher & Jutta & LLNL \\
\hline Fai & George & Department of Energy \\
\hline Favalli & Andrea & Los Alamos National Laboratory \\
\hline Felker & Lisa & EVENT COORDINATOR - LLNL \\
\hline Fox & Morgan & University of California, Berkeley \\
\hline Frandsen & Brian & Air Force Institute of Technology \\
\hline Friedrich & Stephan & Lawrence Livermore National Laboratory \\
\hline Fulsom & Bryan & Pacific Northwest National Laboratory \\
\hline Gert & Godfree & Lawrence Livermore National Laboratory \\
\hline Gibson & Nathan & Los Alamos National Laboratory \\
\hline Goldblum & Bethany & University of California, Berkeley \\
\hline Gooden & Matthew & LANL \\
\hline Gott & Matthew & Argonne National Laboratory \\
\hline Grant & Chris & Boston University \\
\hline Greene & John & Argonne National Laboratory \\
\hline Griffin & Patrick & Sandia National Laboratories \\
\hline Grosskopf & Michael & Los Alamos National Laboratory \\
\hline Guardala & Noel & GWU \\
\hline Hawari & Ayman & NC State University \\
\hline Heilbronn & Lawrence & University of Tennessee \\
\hline Henderson & David & Department of Energy \\
\hline Hornback & Donald & DOE/NNSA/NA-22 \\
\hline Hughes & Richard & LLNL \\
\hline Hutchinson & Jesson & NEN-2: Advanced Nuclear Technology \\
\hline Ingraham & Joanna & Defense Threat Reduction Agency \\
\hline Jandel & Marian & University of Massachusetts, Lowell, MA \\
\hline Jankowski & Keith & Department of Energy Office of Science \\
\hline Johnson & Jeffrey & Oak Ridge National Laboratory \\
\hline Kawano & Toshihiko & Los Alamos National Laboratory \\
\hline Keith & Corey & Los Alamos National Lab \\
\hline Koning & Arjan & International Atomic Energy Agency \\
\hline Koster & $\mathrm{Jim}$ & Los Alamos National Laboratory \\
\hline Krenn & Christopher & Lawrence Livermore National Laboratory \\
\hline Kulp & William & Defense Threat Reduction Agency \\
\hline Laplace & Thibault & University of California, Berkeley \\
\hline Lee & Hye Young & Los Alamos National Laboratory \\
\hline Lewis & Amanda & University of California, Berkeley \\
\hline Lewis & Rebecca & DOE/ NNSA/ NA-22 \\
\hline Little & Robert & LANL \\
\hline Littlejohn & Bryce & Illinois Institute of Technology \\
\hline Loveland & Walter & Oregon State University \\
\hline
\end{tabular}




\begin{tabular}{|c|c|c|}
\hline Lovell & Amy & Los Alamos National Laboratory \\
\hline Lukosi & Eric & University of Tennessee \\
\hline Lyons & Stephanie & Pacific Northwest National Laboratory \\
\hline Mace & Melanie & Defense Threat Reduction Agency \\
\hline Mackney & Daniel & US Air Force \\
\hline Manfredi & Juan & University of California, Berkeley \\
\hline Marchand & Julie & EVENT COORDINATOR - LLNL \\
\hline Marshall & William & Oak Ridge National Laboratory \\
\hline Matheson & Zachary & $\begin{array}{l}\text { National Nuclear Security Administration Office } \\
\text { of Advanced Simulation and Computing }\end{array}$ \\
\hline Matters & David & Defense Threat Reduction Agency \\
\hline Matthews & Eric & UC Berkeley \\
\hline Mauborgne & Marie-Laure & Schlumberger \\
\hline McConchie & Seth & Oak Ridge National Laboratory \\
\hline McCutchan & Elizabeth & Brookhaven National Laboratory \\
\hline Medvedev & Dmitri & BNL \\
\hline Moon & Namdoo & $\begin{array}{l}\text { DHS - Countering Weapons of Mass } \\
\text { Destruction }\end{array}$ \\
\hline Moore & Michael & Pacific Northwest National Laboratory \\
\hline Mumpower & Matthew & LANL \\
\hline Nakae & Les & Lawrence Livermore National Lab \\
\hline Neudecker & Denise & Los Alamos National Laboratory \\
\hline Neufcourt & Leo & Michigan State University \\
\hline Nobre & Gustavo & Brookhaven National Laboratory \\
\hline O'Brien & Ellen & Los Alamos National Laboratory \\
\hline Osborne & Andrew & THE COLORADO SCHOOL OF MINES \\
\hline Parsons & Kent & Los Alamos National Laboratory \\
\hline Peltz & James & NA-114 \\
\hline Percher & Catherine & Lawrence Livermore National Laboratory \\
\hline Perdikakis & Georgios & Central Michigan University \\
\hline Pierson & Bruce & PNNL \\
\hline Potel Aguilar & Gregory & Lawrence Livermore National Laboratory \\
\hline Prasad & Shikha & Texas A\&M University \\
\hline Quirk & Mihaela & Department of Energy \\
\hline Quiter & Brian & Lawrence Berkeley Laboratory \\
\hline Ramos & Christopher & DOE/ NNSA/ NA-22 \\
\hline Ratkiewicz & Andrew & Lawrence Livermore National Laboratory \\
\hline Rearden & Bradley & X-energy \\
\hline Ressler & Jennifer Jo & Lawrence Livermore National Laboratory \\
\hline Rising & Michael & Los Alamos National Laboratory \\
\hline Romano & Catherine & Oak Ridge National Laboratory \\
\hline
\end{tabular}




\begin{tabular}{|c|c|c|}
\hline Rose & Timothy & LLNL \\
\hline Rotsch & David & Argonne National Laboratory \\
\hline Rykaczewski & Krzysztof & Oak Ridge National Laboratory \\
\hline Savard & Guy & Argonne National laborary \\
\hline Schillebeeckx & Peter & European Commission, Joint Research Centre \\
\hline Schunck & Nicolas & Lawrence Livermore National Laboratory \\
\hline Severin & Gregory & Michigan State University \\
\hline Shipbaugh & Calvin & DTRA \\
\hline Sloan & Craig & DOE/ NNSA/ NA-22 \\
\hline Smith & Michael & Oak Ridge National Laboratory \\
\hline Snyder & Lucas & LLNL \\
\hline Sobes & Vladimir & Univerisity of Tennessee \\
\hline Soltz & Ron & LLNL \\
\hline Songzoni & Alejandro & Brookhaven National Laboratory \\
\hline Souders & Andy & Oak Ridge National Laboratory \\
\hline Stave & Sean & DOE/ NNSA/ NA-24 \\
\hline Talou & Patrick & Los Alamos National Laboratory \\
\hline Thompson & Nicholas & LANL \\
\hline Thompson & Jason & NNL \\
\hline Tonchev & Anton & Lawrence Livermore National Laboratory \\
\hline Tovesson & Fredrik & Argonne National Laboratory \\
\hline Trumbull & Timothy & Naval Nuclear Laboratory \\
\hline Valentine & John & Lawrence Berkeley National Laboratory \\
\hline Vanhoy & Jeffrey & United States Naval Academy \\
\hline Venkataraman & Ram & Oak Ridge National Laboratory \\
\hline Verbeke & Jerome & Lawrence Livermore National Laboratory \\
\hline Vermeulen & Christiaan & LANL \\
\hline Vincente Valdez & Pedro & University of California, Berkeley \\
\hline Vogt & Ramona & Lawrence Livermore National Laboratory \\
\hline Voinov & Alexander & Ohio University \\
\hline Voyles & Andrew & University of California, Berkeley \\
\hline Warnick & Kernan & DOE/ NNSA/ NA-22 (PNNL) \\
\hline Wendt & Kyle & Lawrence Livermore National Laboratory \\
\hline Wieselquist & Will & Oak Ridge National Laboratory \\
\hline Winkelbauer & Jack & Los Alamos National Laboratory \\
\hline Wood & Lynn & Pacific Northwest National Laboratory \\
\hline Worrall & Andy & Oak Ridge National Laboratory \\
\hline Wu & Ching-Yen & Lawrence Livermore National Laboratory \\
\hline Yoo & Shinjae & Brookhaven National Lab \\
\hline Zerkle & Michael & Naval Nuclear Laboratory \\
\hline Zhang & Guannan & Oak Ridge National Laboratory \\
\hline
\end{tabular}




\begin{tabular}{lll}
\hline Zhu & Shaofei & NNDC,BNL \\
\hline Zimmerman & Brian & National Institute of Standards and Technology
\end{tabular}

